\title{
A Delta-Sigma Frequency Discriminator with Fractional-N Divider and 3-Bit Quantizer
}

\author{
Jian-Hong Fang \\ M.A.Sc, B.Eng \\ A Thesis Submitted To The Faculty Of \\ Graduate Studies And Research \\ In Partial Fulfilment Of The Requirements \\ For The Degree Of M.A.Sc \\ Department of Electronics \\ Carleton University, Ottawa, Canada \\ (C) Jian-Hong Fang, 2007
}




$\begin{array}{ll}\begin{array}{l}\text { Library and } \\ \text { Archives Canada }\end{array} & \begin{array}{l}\text { Bibliothèque et } \\ \text { Archives Canada }\end{array} \\ \begin{array}{l}\text { Published Heritage } \\ \text { Branch }\end{array} & \begin{array}{l}\text { Direction du } \\ \text { Patrimoine de l'édition }\end{array} \\ \begin{array}{l}\text { 395 Wellington Street } \\ \text { Ottawa ON K1A 0N4 }\end{array} & \begin{array}{l}\text { 395, rue Wellington } \\ \text { Ottana ON K1A ON4 } \\ \text { Canada Oa }\end{array}\end{array}$

Your file Votre référence ISBN: 978-0-494-26986-2 Ourfile Notre référence ISBN: 978-0-494-26986-2

NOTICE:

The author has granted a nonexclusive license allowing Library and Archives Canada to reproduce, publish, archive, preserve, conserve, communicate to the public by telecommunication or on the Internet, loan, distribute and sell theses worldwide, for commercial or noncommercial purposes, in microform, paper, electronic and/or any other formats.

The author retains copyright ownership and moral rights in this thesis. Neither the thesis nor substantial extracts from it may be printed or otherwise reproduced without the author's permission.
AVIS:

L'auteur a accordé une licence non exclusive permettant à la Bibliothèque et Archives Canada de reproduire, publier, archiver, sauvegarder, conserver, transmettre au public par télécommunication ou par l'Internet, prêter, distribuer et vendre des thèses partout dans le monde, à des fins commerciales ou autres, sur support microforme, papier, électronique et/ou autres formats.

L'auteur conserve la propriété du droit d'auteur et des droits moraux qui protège cette thèse. $\mathrm{Ni}$ la thèse ni des extraits substantiels de celle-ci ne doivent être imprimés ou autrement reproduits sans son autorisation.
In compliance with the Canadian

Privacy Act some supporting forms may have been removed from this thesis.

While these forms may be included in the document page count, their removal does not represent any loss of content from the thesis.
Conformément à la loi canadienne sur la protection de la vie privée, quelques formulaires secondaires ont été enlevés de cette thèse.

Bien que ces formulaires aient inclus dans la pagination, il n'y aura aucun contenu manquant. 


\section{Abstract}

The design and implementation of a new Frequency Discriminator (FD) architecture is presented in this thesis. This architecture has finer frequency resolution and lower frequency noise compared to existing architectures. This superior performance is achieved by incorporating a fractional-N divider and a multi-bit quantizer. The new architecture is based on Delta-Sigma $(\Delta \Sigma)$ Modulation techniques and converts instantaneous frequency directly to digital form.

Simulation models were developed in Matlab Simulink to explore the effect of various parameters on performance. The models then were transformed into circuit blocks. The circuit blocks were simulated both at the behavioural and the transistor level. The design was fabricated in $0.18 \mu \mathrm{m}$ TSMC CMOS process.

This FD architecture is suitable for use in high resolution frequency synthesis and frequency demodulation applications. 


\section{Acknowledgements}

I would like to thank Prof. Miles Copeland and Dr. Norm Filiol for their encouragement and guidance over the course of this research and Mr. Tom Riley of Kaben Research Inc. for technical and financial support. This research was funded by CITO and Kaben Research through the Student Internship Program.

Special thanks go to Dr. John Hulse from Institute for Microstructural Science in National Research Council Canada for generously lending his hands on fault analysis and for turning a designer's nightmare to a enjoyable and learning experience.

I also owe thanks to the following individuals for their help in different areas:

Gordon Allan: Digital Design Flow. Yunle Sun: Analog Layout. Mary Ann Riley: Administration of Research Funding.

Finally, I want to thank my lovely wife, Ni Liu, for her continuous and selfless support. As a former world champion in sports acrobatics, she appreciates the hard work and determination both in sports and academic world.

iii 


\section{Contents}

1 Introduction 1

1.1 Motivation ...................... 1

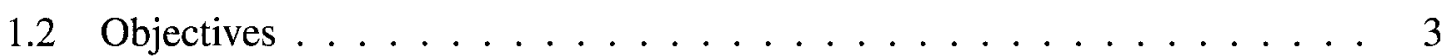

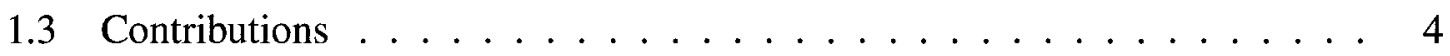

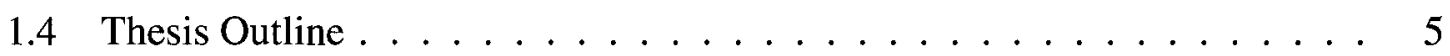

2 Background $\quad 7$

2.1 Frequency Discriminators . . . . . . . . . . . . . 7

2.1.1 Analog Frequency Discriminators ............ 8

$2.1 .2 \quad$ PLL Based FDs . . . . . . . . . . . . . . . . 9

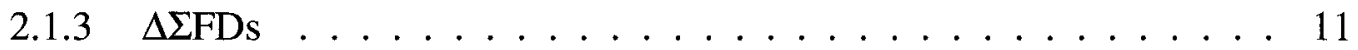

2.1.4 Comparison of Different Types of Frequency Discriminators . . . . 12

$2.2 \Delta \Sigma$ Modulation $\ldots \ldots \ldots \ldots \ldots \ldots \ldots$

2.2.1 Over-Sampling .................... 14

2.2.2 Noise-Shaping ..................... 16

2.3 First-Order $\Delta \Sigma$ Modulators . . . . . . . . . . . . . . . . 18

2.4 Second-Order $\Delta \Sigma$ Modulators . . . . . . . . . . . . . . . . . 20

2.5 Higher-Order $\Delta \Sigma$ Modulators . . . . . . . . . . . . . . 22

iv 


\section{Analysis of a Second-Order $\Delta \Sigma$ FD Having a Fractional-N Divider and a Multi-}

Bit Quantizer 23

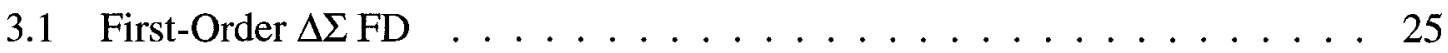

3.1.1 Linear Model for Dual-Modulus Divider . . . . . . . . . . . . 27

3.1.2 Linear Model for a First Order $\Delta \Sigma$ FD . . . . . . . . . . . . . . 29

3.1.3 Performance Evaluation . . . . . . . . . . . . 30

3.2 Second-Order $\Delta \Sigma$ FD with Integer-N Divider and Single-Bit Quantizer . . . 33

3.3 Second-Order FD with Fractional-N Divider . . . . . . . . . . . . . . 37

3.4 A Second-Order $\Delta \Sigma$ FD Using a Fractional-N divider and a Three-Bit Quantizer (Proposed Architecture) . . . . . . . . . . . . . . . . . . 42

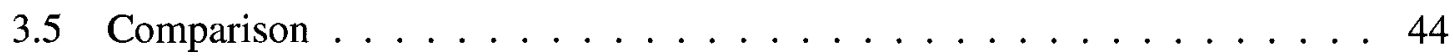

3.6 Effects Of System Parameters on Performance . . . . . . . . . . . 46

3.7 System Noise Analysis . . . . . . . . . . . . . . . . . . . . 48

3.7.1 Quantization Noise Transfer Function . . . . . . . . . . . 50

$3.7 .2 f_{\text {in }}$ Noise Transfer Function $\ldots \ldots \ldots \ldots$

$3.7 .3 f_{\text {ref }}$ Noise Transfer Function . . . . . . . . . . . 50

3.7.4 Divider Noise Transfer Function . . . . . . . . . . . . 50

3.7.5 PFD and CP Noise Transfer Function . . . . . . . . . . . 51

3.7.6 Integrator Noise Transfer Function . . . . . . . . . . . . 51

3.7.7 Quantizer Noise Transfer Function . . . . . . . . . . . . . . 51

3.8 Circuit Performance Improvement Techniques . . . . . . . . . . . . 52

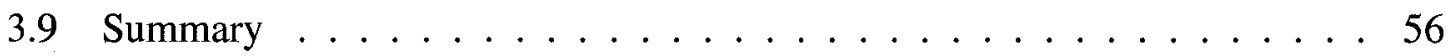

4 Circuit Design and Simulation Results $\quad 57$

$4.1 f_{\text {in }}$ and $f_{r e f}$ Input Buffers $\ldots \ldots \ldots \ldots \ldots$

4.1.1 Simulation Results . . . . . . . . . . . . . . . . 6 61 
4.1.2 Phase Noise Performance ............... 62

4.2 Programmable Multi-Modulus Divider . . . . . . . . . . . . . . . 64

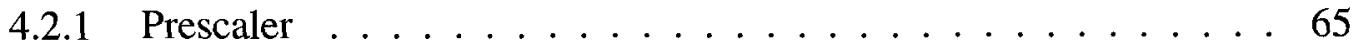

4.2 .2 M-Counter .......................... 66

4.2 .3 A-Counter .......................... 68

4.2.4 Decoding of M-Counter States . . . . . . . . . . . . . . . 68

4.2 .5 Simulation of MMD ................... 71

4.3 Phase-Frequency Detector .................. 72

4.3.1 PFD Noise Performance . . . . . . . . . . . . . 75

4.4 Charge Pump ... . . . . . . . . . . . . . . 76

4.4.1 PFD/CP Noise Performance . . . . . . . . . . . . . 80

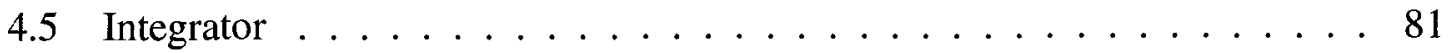

4.5 .1 Noise Performance .................. 84

4.6 3-Bit Quantizer . . . . . . . . . . . . . . . 85

4.6 .1 Comparator Design . . . . . . . . . . . . . 86

4.6 .2 Simulation Results . . . . . . . . . . . . . . 88

4.6 .3 Noise Performance . . . . . . . . . . . . . . . . . . 90

4.7 Digital Feedback Block (DFB) . . . . . . . . . . . . . . . 91

4.7 .1 Encoder ............................ 91

$4.7 .2 \quad 2-Z^{-1}$ block ....................... 91

4.7.3 Residual Accumulator . . . . . . . . . . . . . 92

4.7.4 Data Weighted Averaging Block . . . . . . . . . . . . 92

4.7 .5 Simulation Result . . . . . . . . . . . . . . . . . . . . 94

4.8 Serial Interface $\ldots \ldots \ldots 5$

4.9 Overall Performance . . . . . . . . . . . . . . . . 99

$\mathrm{vi}$ 
$\begin{array}{lll}5 & \text { Measurements } & 104\end{array}$

5.1 Chip Layout and Test Setup . . . . . . . . . . . . . . . . . . 104

5.2 Fabrication and Fault Analysis . . . . . . . . . . . . . . 107

6 Conclusions and Future Work 116

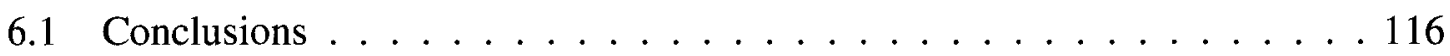

6.1 .1 Summary ............................. 116

6.1.2 Advantages Over Existing $\Delta \Sigma$ FD Designs . . . . . . . . . . . . . 119

6.2 Future Work . . . . . . . . . . . . . . . . . . . 119 


\section{List of Figures}

1.1 A Conventional PLL Based Frequency Synthesizer . . . . . . . . . . . . 1

1.2 A Frequency Discriminator Based Frequency Synthesizer . . . . . . . . . . 2

2.1 Narrow Bandpass Filter Used as a FD . . . . . . . . . . . . . 8

2.2 FD with Improved Linearity $\ldots \ldots . \ldots$

2.3 Slope Demodulator . . . . . . . . . . . . . . . . 9

2.4 PLL Used as a FD . . . . . . . . . . . . . . . . . . . 10

2.5 Model of a First-Order PLL Frequency Discriminator . . . . . . . . . . . 11

2.6 Block Diagram of First-Order $\Delta \Sigma$ FD . . . . . . . . . . . . 11

2.7 A Block Diagram for a First-Order DSM . . . . . . . . . . . . . 13

2.8 Illustration of Sampling and Quantization $\ldots \ldots \ldots \ldots$

2.9 Quantizer Linear Model . . . . . . . . . . . . . . . . . 15

2.10 In-band Noise Decreases with Increasing Sampling Frequency . . . . . . 16

2.11 Noise Spectral Density before and after Shaping . . . . . . . . . . . 17

2.12 Linear Model of a First Order DSM . . . . . . . . . . . . 18

2.13 Linear Model of a Second Order DSM . . . . . . . . . . . . . 20

3.1 Block Diagram for a First-Order $\Delta \Sigma$ FD . . . . . . . . . . 25

3.2 Timing Diagram for a First-Order $\Delta \Sigma$ FD . . . . . . . . . . 25

viii 
3.3 Phase Ramps for $f_{r e f}$ and $f_{d i v} \quad \ldots \ldots \ldots \ldots \ldots \ldots \ldots \ldots$

3.4 Linear Model for Dual-Modulus Divider . . . . . . . . . . . . . . . . . 29

3.5 Linear Model for a First-Order $\Delta \Sigma$ FD . . . . . . . . . . . . . . . . . . . . 29

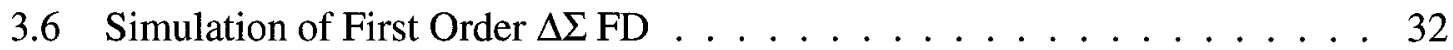

3.7 Second Order $\Delta \Sigma$ FD Block Diagram . . . . . . . . . . . . . . 33

3.8 Second Order $\Delta \Sigma$ FD Linear Model $\ldots \ldots \ldots \ldots$

3.9 Matlab Simulation Result for the Second Order $\Delta \Sigma$ FD . . . . . . . . . 35

3.10 An Example of Phase Error Cancellation in Fractional-N Frequency Synthesis 37

3.11 Block Diagram for a Second-Order $\Delta \Sigma$ FD With Fractional-N Divider . . . 38

3.12 Linear Model of Second Order $\Delta \Sigma$ FD with Fractional-N Divider . . . . . . 39

3.13 Integer-N versus Fractional-N Divider Comparison $\ldots \ldots \ldots$. . . . . 41

3.14 Second-Order $\Delta \Sigma$ FD with Fractional-N Divider and Multi-Bit Quantizer . . 42

3.15 Linear Model for Figure $3.14 \ldots \ldots \ldots$

3.16 SNR vs Input Frequency Deviation . . . . . . . . . . . . . . 45

3.17 Linear Model of 2nd-Order Fractional-N $\Delta \Sigma$ FD . . . . . . . . . . . . . . 48

3.18 Noise Transfer Function at Various Circuit Nodes . . . . . . . . . . 52

3.19 CP Output with (a) no DWA, and (b) DWA . . . . . . . . . 53

3.20 DWA for Eight Elements - Input Sequence: 011, 100 and 010 . . . . . . 54

3.21 Comparison between no CP Mismatch, CP Mismatch with and without DWA 56

4.1 System Block Diagram . . . . . . . . . . . . . . . . . . . 57

$4.2 \Delta \Sigma$ FD Design Flow and Tools Used $\ldots \ldots \ldots \ldots$

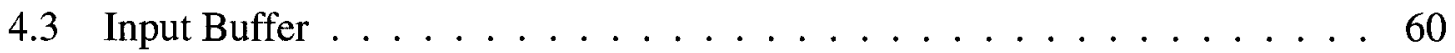

$4.4 f_{r e f}$ Input Buffer Operation $\ldots \ldots \ldots \ldots \ldots \ldots \ldots \ldots \ldots$

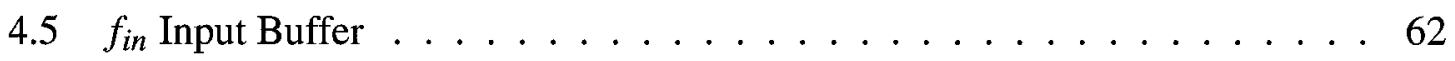

4.6 Output Phase Noise of $f_{r e f}$ Buffer $\ldots \ldots \ldots \ldots \ldots \ldots$

ix 
4.7 Output Phase Noise of $f_{\text {in }}$ Buffer . . . . . . . . . . . . . 63

4.8 Programmable Multi-Modulus Divider . . . . . . . . . . . . . . 64

4.9 Programmable MMD Timing . . . . . . . . . . . . . . . . 65

4.10 Schematic for Prescaler . . . . . . . . . . . . . . . . . 66

4.11 Schematic for M-Counter . . . . . . . . . . . . . . . . 67

4.12 Schematic for A-Counter . . . . . . . . . . . . . . 68

4.13 Mapping of M-counter States to MMD Timing Signals . . . . . . . . . 70

4.14 RTL Test Bench Simulation Results . . . . . . . . . . . . . . 71

4.15 A schematic for a PFD . . . . . . . . . . . . 72

4.16 PFD with DC offset . . . . . . . . . . . . . . . 73

4.17 PFD Transition Simulation: Fref Leading Fdiv _ . . . . . . . . . . 74

4.18 PFD Transition Simulation: Fref Lagging Fdiv . . . . . . . . . . . . 75

4.19 Schematic for one Charge Pump element . . . . . . . . . . . . . 77

4.20 Charge Pump Operation . . . . . . . . . . . . . . . . 79

$4.21 \mathrm{PFD} / \mathrm{CP}$ Output-Referred Noise $\ldots \ldots$. . . . . . . . . . . 80

4.22 (a) a Passive Integrator and (b) an Active Integrator . . . . . . . . . . 81

4.23 Schematic for a Active Integrator . . . . . . . . . . . . 82

4.24 Integrator Transient Response . . . . . . . . . . . . . . . 83

4.25 Integrator Output Referred Noise for Lock Condition . . . . . . . . . . 84

4.26 An Array of Eight Comparators Used as a 3-bit Quantizer . . . . . . . . . 85

4.27 Comparator Schematic . . . . . . . . . . . . . . . 87

4.28 Quantizer Simulation . . . . . . . . . . . . . . . . 89

4.29 Quantizer Input-Referred Noise . . . . . . . . . . . . . . . 90

4.30 The Digital Feed Back Block . . . . . . . . . . . . . . . . . 91

4.31 Residual Accumulator . . . . . . . . . . . . . . . . . 92 
4.32 Block Diagram for DWA implementation . . . . . . . . . . . 93

4.33 DFB RTL Simulation Result . . . . . . . . . . . . . . . . . . 94

4.34 Implementation of Serial Interface . . . . . . . . . . . . . . 95

4.35 Serial Interface Timing . . . . . . . . . . . . . . . . . . 97

4.36 Serial Interface RTL Simulation _ . . . . . . . . . . . . . . 98

4.37 System Simulation with Verilog-A and Verilog . . . . . . . . . . 99

4.38 System Simulation (PFD Modelled at the Transistor Level) . . . . . . . . 100

4.39 System Simulation (CP Modelled at the Transistor Level) . . . . . . . . . 100

4.40 System Simulation (Quantizer Modelled at the Transistor Level) . . . . . 101

4.41 System Frequency Noise Performance . . . . . . . . . . . . . . . 102

4.42 System Phase Noises Performance . . . . . . . . . . . . . . . 103

5.1 Chip Layout . . . . . . . . . . . . . . . . . . . . 105

5.2 Test Setup . . . . . . . . . . . . . . . . . . 106

5.3 Block Diagram for a DEI System [HFSN04] . . . . . . . . . . . . 108

5.4 Test setup with DEI System . . . . . . . . . . . . . . . . . . . 109

5.5 Locations for DEI Measurements . . . . . . . . . . . . . . . . 110

5.6 DEI Images. Left: RESET is inactive and Right: RESET is active . . . . 111

5.7 Time-Correlated Waveform When RESET is active . . . . . . . . . . . . 112

5.8 Time-Correlated Waveform When RESET is inactive . . . . . . . . . . . . 112

5.9 Divider Waveform . . . . . . . . . . . . . . . . . 114

5.10 Time-Correlated Waveform When RESET=OFF . . . . . . . . . . 115 


\section{List of Tables}

2.1 Comparison of Different Types of Frequency Discriminators . . . . . . . . 12

$3.1 \Delta \Sigma$ FD Architecture Performance Comparison . . . . . . . . . . . . . . . . 44

3.2 Simulation Result for Different Combinations of $f_{r e f}$ and $n \ldots \ldots 4$

4.1 Prescaler State Table . . . . . . . . . . . . . . 66

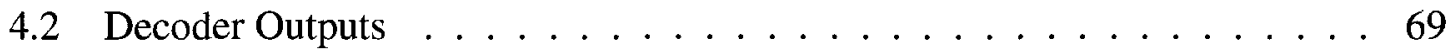

4.3 CP Output Current vs Control Bits . . . . . . . . . . . . 78

4.4 Static Control Signals . . . . . . . . . . . . . . . 95

4.5 Serial Interface Signals . . . . . . . . . . . . . . . . . 96

4.6 System SpectreVerilog Simulations . . . . . . . . . . . . . . . . . . . . 99

5.1 External Pin DC Bias Level Measurement . . . . . . . . . . . . . . . . . 107 


\section{Chapter 1}

\section{Introduction}

\subsection{Motivation}

Frequency synthesizers are an essential component in wireless communication devices. It is very difficult to find a transceiver today that does not employ at least one if not several frequency synthesizers.

Phase Locked Loop (PLL) based frequency synthesis is the most commonly used method of generating stable high frequencies. The architecture of a conventional PLL based frequency synthesizer is shown in Fig.1.1.

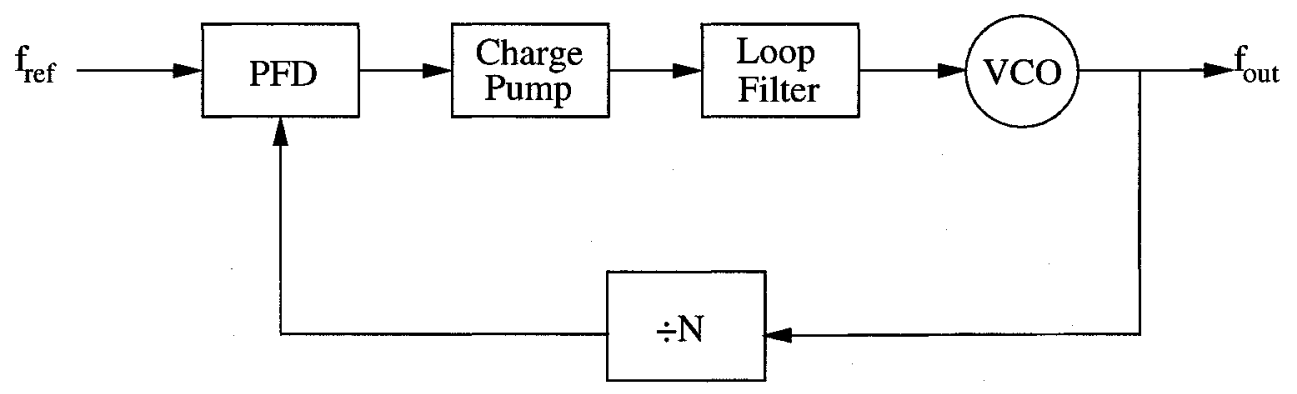

Figure 1.1: A Conventional PLL Based Frequency Synthesizer 
The system compares the frequencies of two signals (one is the reference clock, $f_{\text {ref }}$, the other one is the divided-down version of VCO output frequency, $f_{\text {out }}$ ) and produces an error signal which is proportional to the difference between the phases of these two signals. The error signal, after being processed, is used to drive the Voltage-Controlled Oscillator (VCO). The output frequency from the VCO passes through a frequency divider back to the input of the phase comparator, producing a negative feedback loop. If the reference clock or the division ratio, $N$, changes, the error signal will increase, driving the VCO frequency in the opposite direction to reduce the error. Thus, the VCO frequency is always locked to $N$ times the reference clock.

A second generation synthesizer architecture [Bax94] is shown in Fig.1.2.

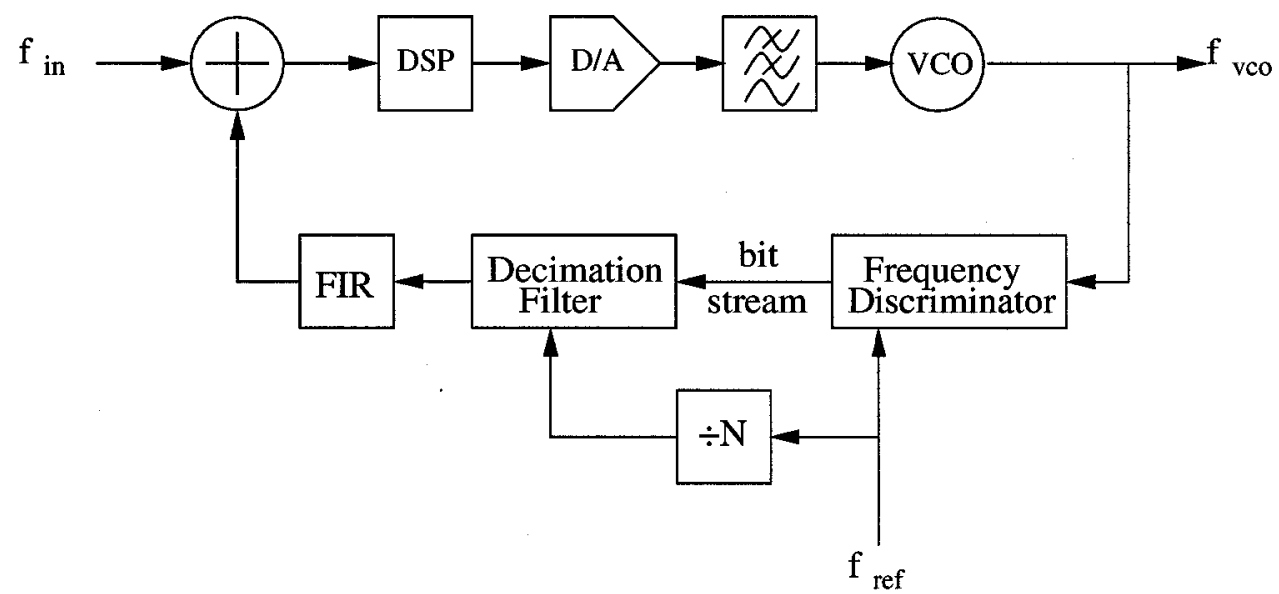

Figure 1.2: A Frequency Discriminator Based Frequency Synthesizer

The architecture shown in Fig.1.2 is based on an FD in the feedback path, which measures the VCO frequency and produces results in digital form. The FD functions as a frequency demodulator and analog-to-digital converter, and replaces the divider and phase detector in a conventional PLL synthesizer. Early conversion of the VCO frequency into digital form permits much of the remaining loop to be realized using Digital Signal Pro- 
cessing (DSP) techniques. Since most of the loop is digital, the loop dynamics can be changed by re-programming the loop filter on the fly to suit different application needs. Such flexibility is the key to multi-mode transceivers.

Operation of this synthesizer architecture is similar to conventional PLL based ones. The FD bit-stream is decimated and scaled to give a measure of the VCO frequency. This digital value is compared to the desired reference frequency word to produce an error signal. The error signal is digitally filtered and converted to an analog signal to drive the VCO.

Th FD is the key component in this architecture. The remainder of this thesis focuses on the analysis, design and implementation of an improved version of the second-order $\Delta \Sigma$ FD first introduced in [Bax94]. It is hoped that the knowledge gained will allow for further improvements to high performance frequency synthesis.

\subsection{Objectives}

The objectives of this thesis are:

1. To analyze the $\Delta \Sigma$ frequency discriminator presented in [Bax94] and propose improvements.

2. Incorporate a fractional- $\mathrm{N}$ divider into the architecture presented in [Bax94]. This fractional divider should not require highly precise analog circuits and have a fractionality of $1 / 8$. Inclusion of this divider should result in $18 \mathrm{~dB}$ decrease in quantization noise compared to [Bax94].

3. Replace the single-bit quantizer with a multi-bit quantizer in order to maintain the full-scale input range of the discriminator.

4. Analyze the new architecture using Matlab/Simulink. 
5. Implement and test the architecture at the transistor level in a 0.18 um CMOS process.

\subsection{Contributions}

The contributions made in this thesis, to the research area of high performance frequency measurement and frequency synthesis, are the following:

1. The design and analysis of a new architecture for a $\Delta \Sigma$ frequency discriminator that makes use of a novel fractional divider. This discriminator measures the instantaneous frequency of an input signal and converts it directly into digital form. Use of a fractional divider with a step size of $1 / 8$ results in $18 \mathrm{~dB}$ less total quantization noise power in the output bitstream, compared to previous implementations. This reduces the decimation filter requirements after the discriminator and improves the SNR for small deviation FM signals. The fractional divider makes use of a Data Weighted Averaging (DWA) algorithm to apply first order noise shaping to mismatch errors introduced in the divider elements. The end result is a second-order $\Delta \Sigma$ Frequency Discriminator with greatly improved dynamic range compared to previous architectures, providing higher sensitivity to low deviation frequency modulated signals.

2. A Matlab/Simulink model for the discriminator was designed and used to predict performance and to validate the concept of the new architecture. A linear analysis of the architecture was performed in order to create a model for analysis purposes. The model was used to determine the transfer function of the signal and various noise sources to the output of the discriminator. These results are summarized in [FFRC07] and will be presented at ISCAS 2007, in New Orleans, USA.

3. A transistor level design for the new discriminator architecture was created, simu- 
lated and fabricated in the TSMC $0.18 \mu \mathrm{m}$ CMOS process. Simulations were performed, at the transistor level, for each block. The noise predictions from these simulations were applied to the transfer functions determined in 2, and used to predict the SNR of the final design. Phase noise performance indicates a state of the art in-band BFM of -200 at $20 \mathrm{kHz}$ offset. In order to help ensure functionality of the final design, each block was either modelled as a Verilog, Verilog-A or a transistor level circuit. This allowed for SpectreVerilog simulations of the architecture in closed loop, something which would not have been practical at full transistor level.

4. Due to an error in the Output Drivers, provided by the foundry, it was not possible to observe many of the key outputs of the fabricated circuit in the laboratory. As a result of this fault, a research relationship was formed with John Hulse of the NRC and MuAnalysis Inc. to prove the validity of using Dynamic Electroluminescence Imaging (DEI) measurements to debug high performance CMOS circuits. DEI is non-invasive, non-destructive and has a bandwidth approaching $100 \mathrm{GHz}$. This means that very fast signals can be observed without unduly loading or otherwise interfering with the circuitry under test. Using this technique, the feedback divider operation was confirmed. Results of this collaborative research effort have been submitted for publication at CSTC 2007.

\subsection{Thesis Outline}

The remainder of this thesis is organized as follows:

In Chapter 2, several different types of frequency discriminators are reviewed. $\Delta \Sigma$ Modulation techniques are briefly described. Analysis methods for $\Delta \Sigma$ Modulators are also presented. Finally, the $\Delta \Sigma$ FD is introduced: first-order $\Delta \Sigma$ FD, followed by the exten- 
sion to second-order, and finally, second-order with a fractional-N divider and a multi-bit quantizer.

In Chapter 3, the detailed architecture design of the new $\Delta \Sigma$ FD is presented. The various design parameters and their effects on performance are examined closely. Some techniques used to overcome potential circuit imperfections are also explained. Matlab Simulink simulations are performed at this level.

Chapter 4 explains the partitioning of the system at the circuit level. The different design methods/flows for the analog and digital parts of circuits are described. The functionality and specifications for each block are verified through simulation at the behavioural level as well as at the transistor level.

Chapter 5 presents the measurement data from the fabricated chip and the results of DEI testing.

Chapter 6 presents conclusions and suggestions for further work in this area. 


\section{Chapter 2}

\section{Background}

In this chapter, a brief review of FDs and DSM techniques is presented.

\subsection{Frequency Discriminators}

Frequency Discriminators (FDs) convert instantaneous frequency, taking the form of a sum of carrier and modulation components, as expressed in Eq.2.1, into a proportional voltage. The voltage amplitude at the output of a FD may take either analog or digital form, but its changes always follow, in direct proportion, those of the input frequency.

$$
f_{\text {in }}(t)=f_{\text {nom }}+k_{f} s(t)
$$

where $f_{i n}(t)$ is the instantaneous frequency of the input frequency, $f_{\text {nom }}$ the constant nominal carrier frequency, and $k_{f}$ is the proportionality constant relating frequency changes to amplitude values of $s(t)$. 


\subsubsection{Analog Frequency Discriminators}

A simple analog frequency discriminator can be built using a narrow band filter as shown in Fig.2.1.

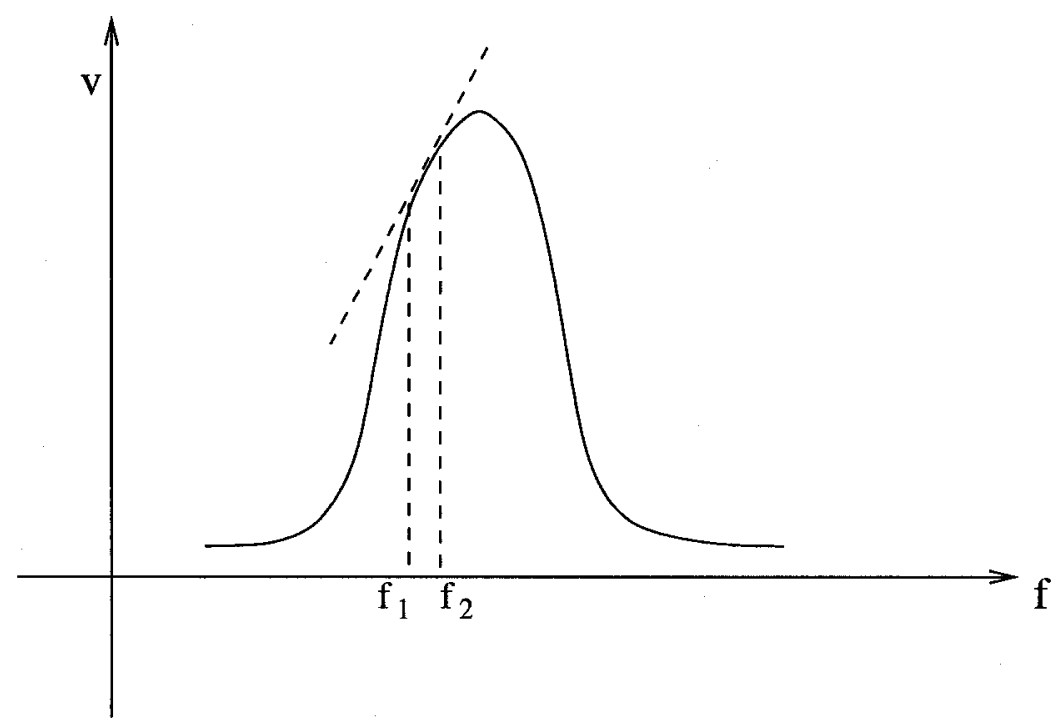

Figure 2.1: Narrow Bandpass Filter Used as a FD

Within a limited range, the voltage is linearly proportional to the frequency. This range of linearity may be improved by subtracting a shifted version of the narrow-band filter's output from the non-shifted version [Rod96], as illustrated in Fig.2.2.

A slope demodulator, depicted in Fig.2.3, is used to implement the improved linearity. It is designed in such way that the even powers in the Taylor series expansion of the filter roll-off function are eliminated and it becomes an odd function of input frequency. The upper half of the output winding of the transformer and $C_{1}$ are tuned to $f_{a}$, and the lower half of the winding and $C_{2}$ are tuned to $f_{b}$. 


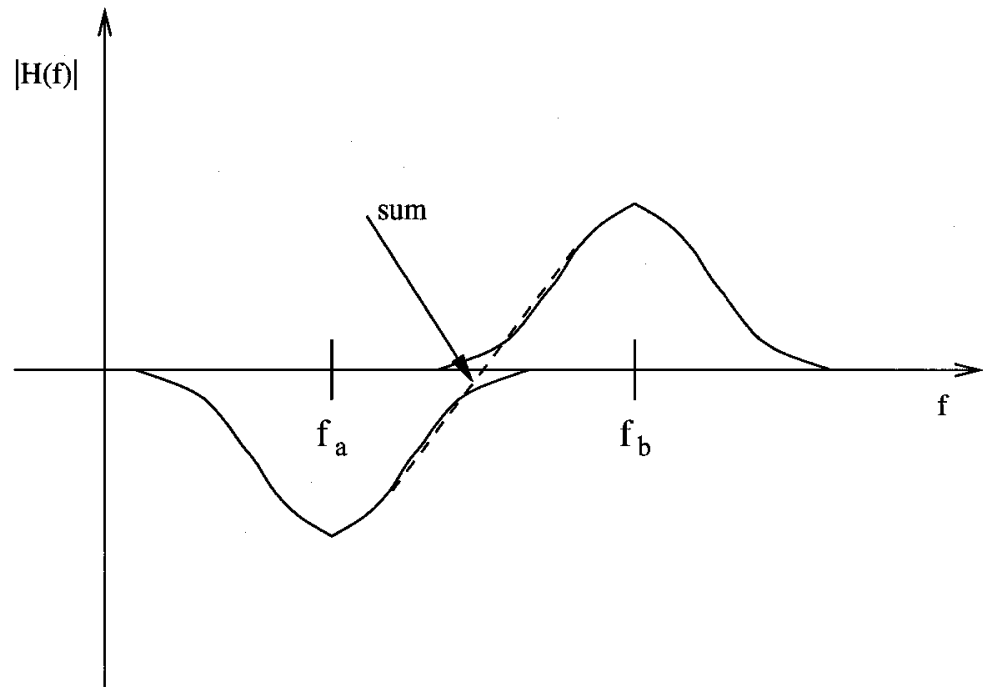

Figure 2.2: FD with Improved Linearity

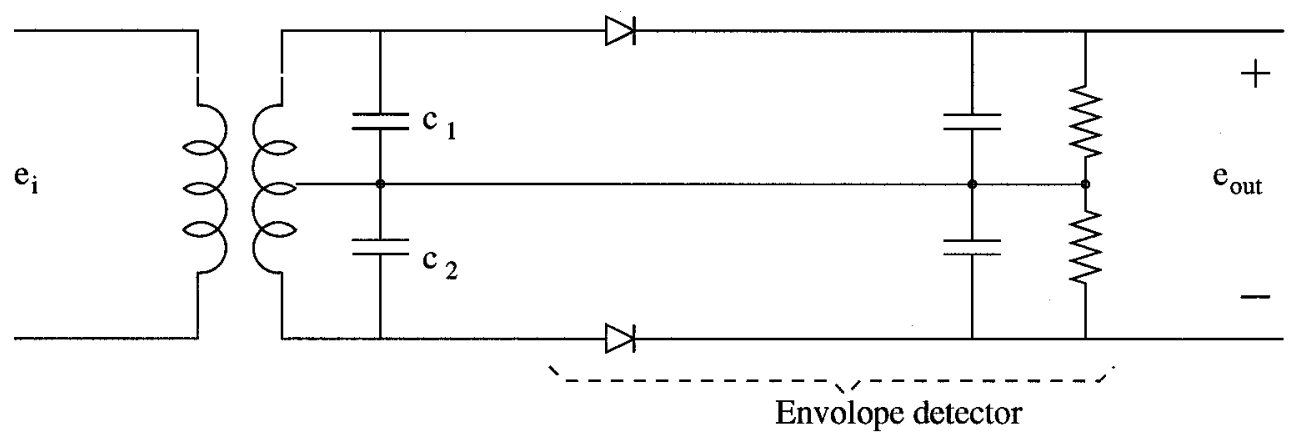

Figure 2.3: Slope Demodulator

\subsubsection{PLL Based FDs}

A PLL can also be used as a frequency discriminator. When the input signal is frequency modulated, the loop-generated error signal will, under certain conditions, be a duplicate of the input modulating signal. The first-order analogue PLL, shown in Fig 2.4, provides an example of this method. 


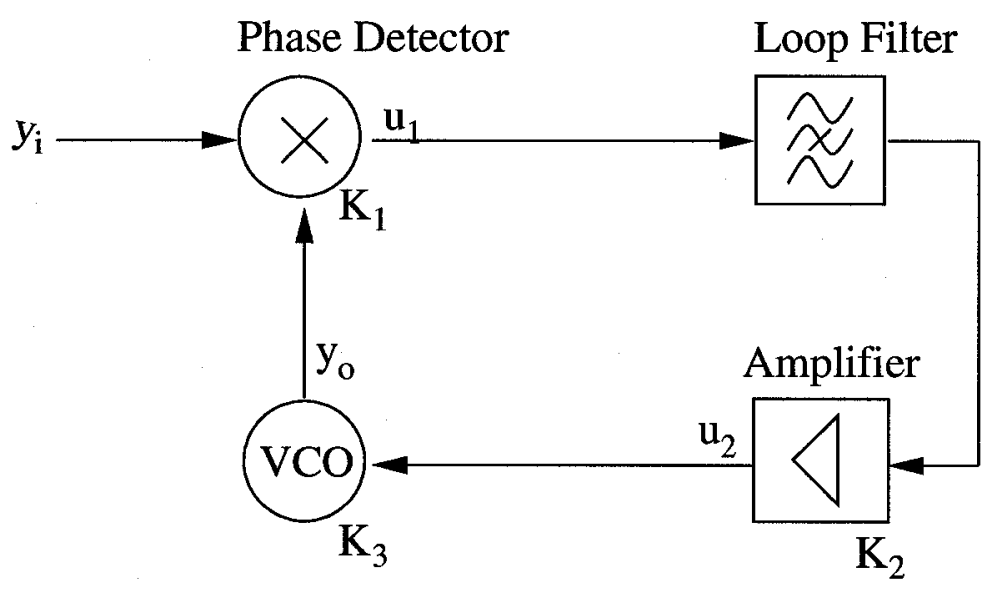

Figure 2.4: PLL Used as a FD

The input signal, $y_{i}(t)$, is frequency modulated by a sinusoidal signal of frequency $\Omega$ and maximum deviation $\Delta f_{i}$. The instantaneous frequency of $y_{i}(t)$ is:

$$
f(t)=f_{c}+\Delta f \sin (\Omega t+\theta)
$$

$y_{i}(t)$ is given by:

$$
y_{i}(t)=A \sin \left(2 \pi f_{c} t+\frac{2 \pi \Delta f}{\Omega} \cos (\Omega t+\theta)\right)
$$

When the phase detector is operating in its linear region, its output is given by:

$$
u_{1}(t)=K_{1} \frac{2 \pi \Delta f}{\sqrt{\left(K_{1} K_{2} K_{3}\right)^{2}+\Omega^{2}}} \sin \left(\Omega t+\theta+\frac{\pi}{2}-\operatorname{Arctan} \frac{\Omega}{K_{1} K_{2} K_{3}}\right)
$$

$u_{1}(t)$ is a duplicate of the modulating signal of Eq 2.2, differing only by a multiplicative constant and low-pass filtering with the time constant $1 /\left(K_{1} K_{2} K_{3}\right)$. A model which corresponds to this functionality is shown in Fig.2.5. 


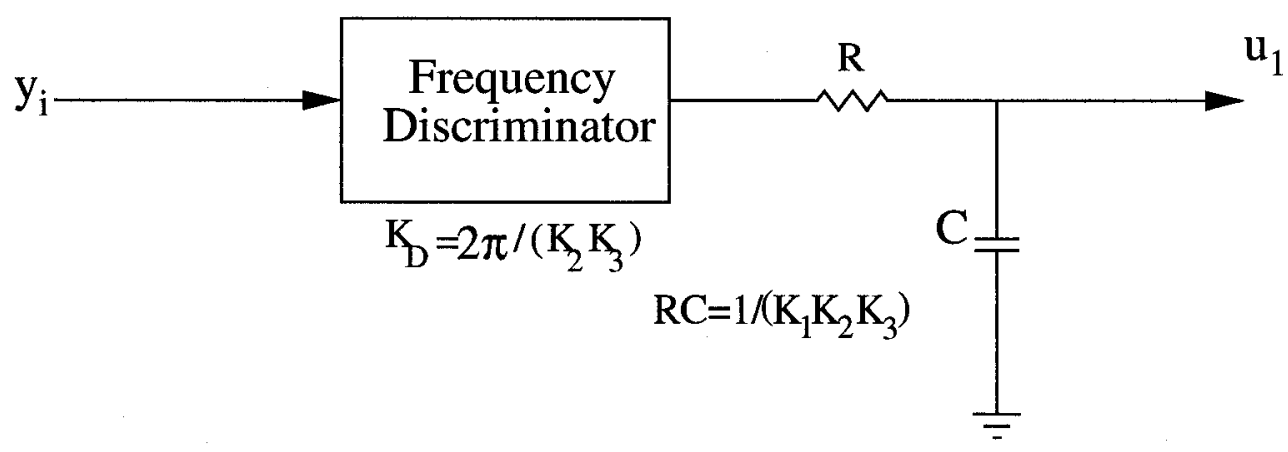

Figure 2.5: Model of a First-Order PLL Frequency Discriminator

\subsection{3 $\Delta \Sigma$ FDs}

A $\Delta \Sigma$ FD architecture introduced in [BC94] converts the instantaneous frequency of an input signal into a digital bit stream having a high SNR in the band of interest. The block diagram for a first-order $\Delta \Sigma$ FD is shown in Fig.2.6.

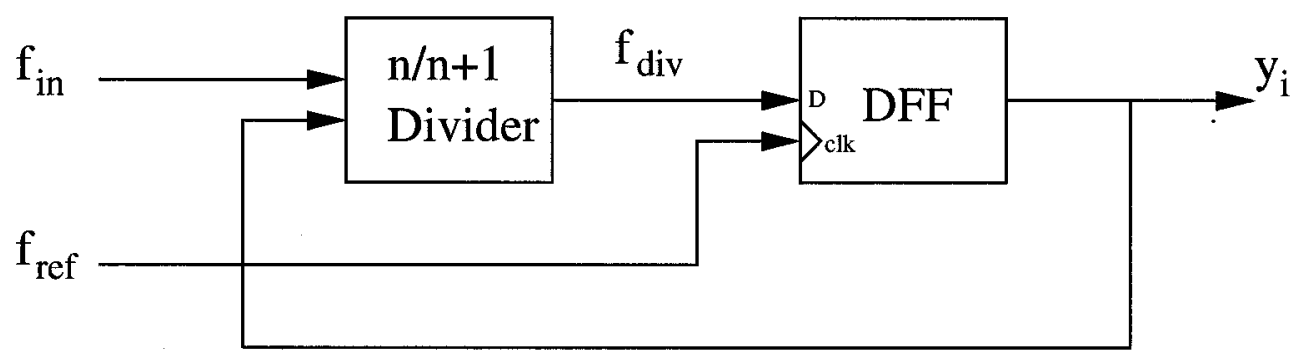

Figure 2.6: Block Diagram of First-Order $\Delta \Sigma$ FD

The term $\Delta \Sigma$ is used here because what this FD does for input frequency is what a $\Delta \Sigma$ A/D modulator does for input voltage. Therefore, all of the knowledge accumulated about conventional DSMs can be applied to the analysis of $\Delta \Sigma$ FDs. In the next section, a review of conventional DSMs is presented. A detailed analysis of $\Delta \Sigma$ FDs is presented in Chapter 3. 


\subsubsection{Comparison of Different Types of Frequency Discriminators}

A comparison of the main performance parameters for the three different types of FDs presented in the previous sections is given in Table. 2.1. It can be seen from the table that the $\Delta \Sigma$-based discriminator performs well for the parameters listed.

\begin{tabular}{|c|c|c|c|}
\hline Parameter & Bandpass Filter & PLL-Based & $\Delta \Sigma$-Based \\
\hline Frequency Input Range & poor & fair & good \\
\hline Frequency Resolution & poor & good & good \\
\hline Linearity & poor & good & good \\
\hline Dynamic Range & poor & fair & good \\
\hline Output Format & analog & analog & digital \\
\hline Phase Continuity & no & yes & yes \\
\hline
\end{tabular}

Table 2.1: Comparison of Different Types of Frequency Discriminators 


\section{$2.2 \Delta \Sigma$ Modulation}

$\Delta \Sigma$ Modulation (DSM) was first introduced in 1962 [IM62] and has been used in applications such as digital phones, digital audio, instrumentation and frequency synthesis [NST96]. The block diagram for an A/D converter using first-order DSM is shown in Fig.2.7.

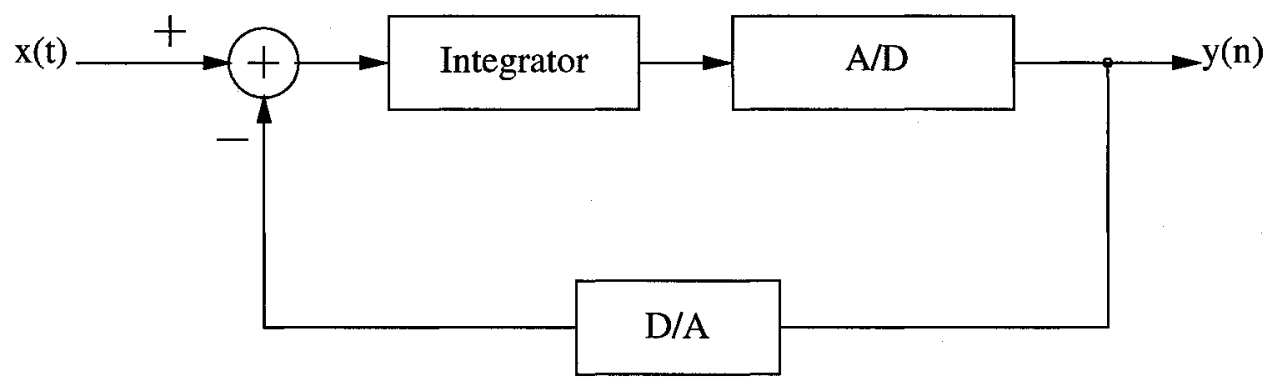

Figure 2.7: A Block Diagram for a First-Order DSM

This type of modulator exploits over-sampling and noise-shaping techniques. Oversampling compensates for coarse quantization with faster sampling speeds and converts a requirement for precise analogue components to one for high speed digital signal processing, thus easing the designer's burden. Noise-shaping is a method for shifting quantization noise to higher frequencies where it is easily suppressed by simple low-pass filtering. The resulting output is an accurate replica of the original analog input with the addition of shaped and filtered quantization noise.

An important difference between a conventional $\mathrm{A} / \mathrm{D}$ converter and a $\Delta \Sigma \mathrm{A} / \mathrm{D}$ converter lies in the correspondence between the input and output sample values. With a conventional $\mathrm{A} / \mathrm{D}$, there is a one-to-one correspondence between the input and output sample values. In contrast, no such correspondence exists in the case of $\Delta \Sigma \mathrm{A} / \mathrm{D}$ converter: for any given point in time, the output sample values doesn't represent the input value at that point, but 
rather it is the time-averaged value of the output that represents the input value. 


\subsubsection{Over-Sampling}

Sampling and Quantization are used to convert a continuous analog signal into series of digital words. The Nyquist Sampling Theorem requires the sampling frequency, $f_{s}$, to be at least twice the maximum signal frequency, $f_{\max }$, in order for the original analogue signal be faithfully recovered from the series of samples.

When converting from an analog signal to a digital signal, error is unavoidable. An analog signal is continuous, with ideally infinite precision, while the digital signal's precision is dependent upon the quantization resolution. The series of differences between actual analog values and their approximated digital values makes up what is called quantization noise. Quantization noise, $e$, is illustrated in Fig.2.8. The linear model for a quantizer is shown in Fig.2.9.

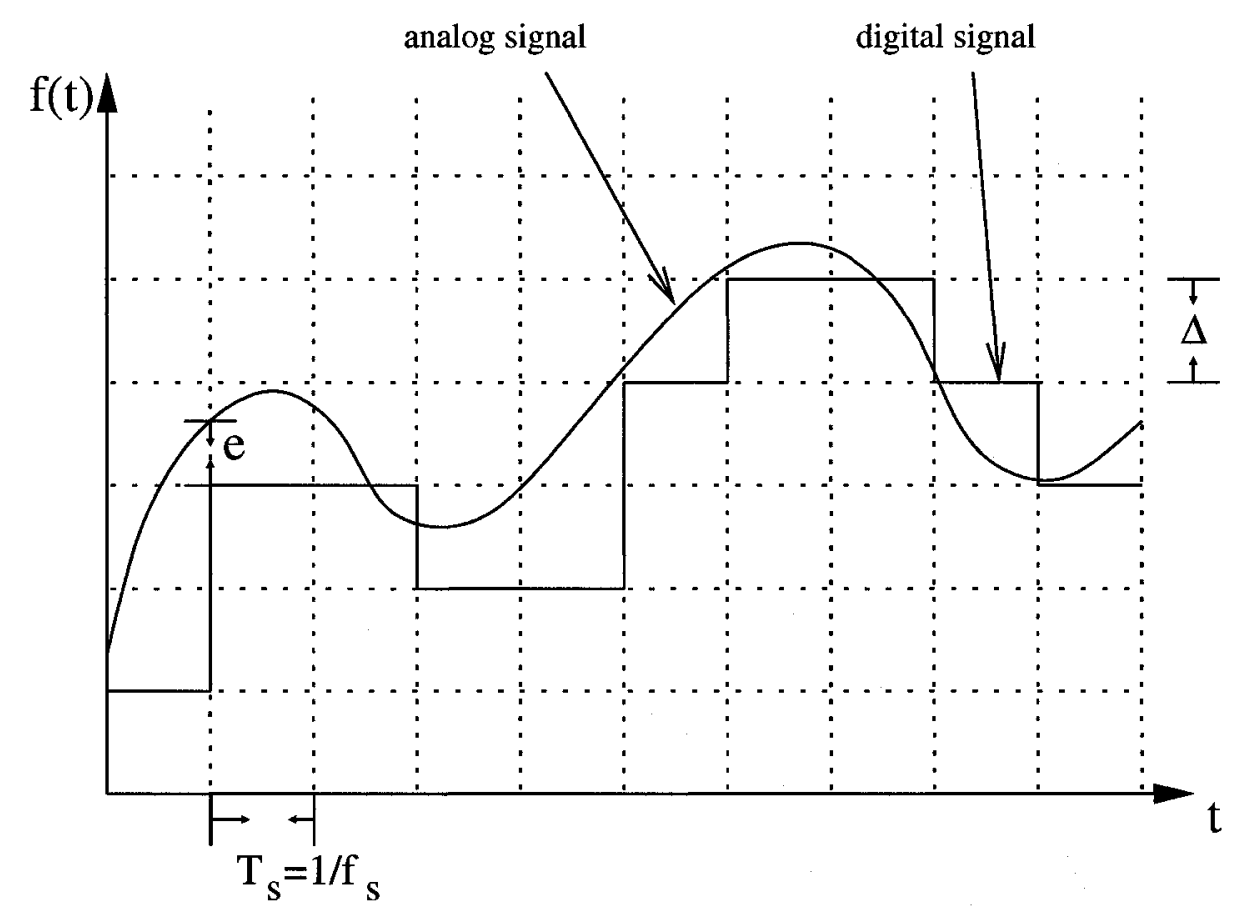

Figure 2.8: Illustration of Sampling and Quantization 


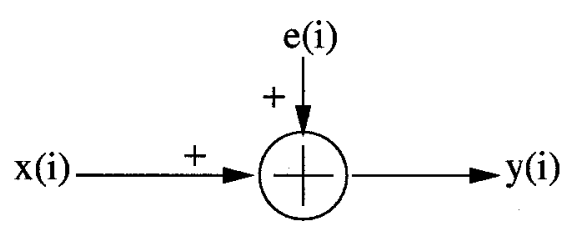

Figure 2.9: Quantizer Linear Model

If $e$ is regarded as having equal probability of lying anywhere in the range $\pm \frac{\Delta}{2}$, where $\Delta$ is the quantization step, the value of the total error power is given by

$$
e_{r m s}^{2}=\frac{1}{\Delta} \int_{-\Delta / 2}^{\Delta / 2} e^{2} d e=\frac{\Delta^{2}}{12}
$$

For a given sampling frequency $f_{s}$, the spectral density of the sampled noise is

$$
E(f)=\sqrt{\frac{e_{r m s}^{2}}{f_{s} / 2}}=\sqrt{2} \frac{e_{r m s}}{\sqrt{f_{s}}}
$$

The quantization noise power that falls into a signal band $0 \leq f<f_{\max }$ is given by

$$
n_{o}^{2}=\int_{0}^{f_{\max }} E^{2}(f) d f=e_{r m s}^{2} \frac{2 f_{\max }}{f_{s}}
$$

Oversampling, the situation where $f_{s}>2 f_{\text {max }}$, has the effect of spreading a fixed amount of quantization noise power over a broader range of frequencies which is above the Nyquist frequency. The consequence of this is a reduction in the noise power in a given fixed bandwidth. In fact, each doubling of the sampling frequency halves the noise power appearing in this band. That is, it reduces the noise power available to contaminate the reconstructed analogue signal by one half. A graphical illustration is shown in Fig.2.10. 

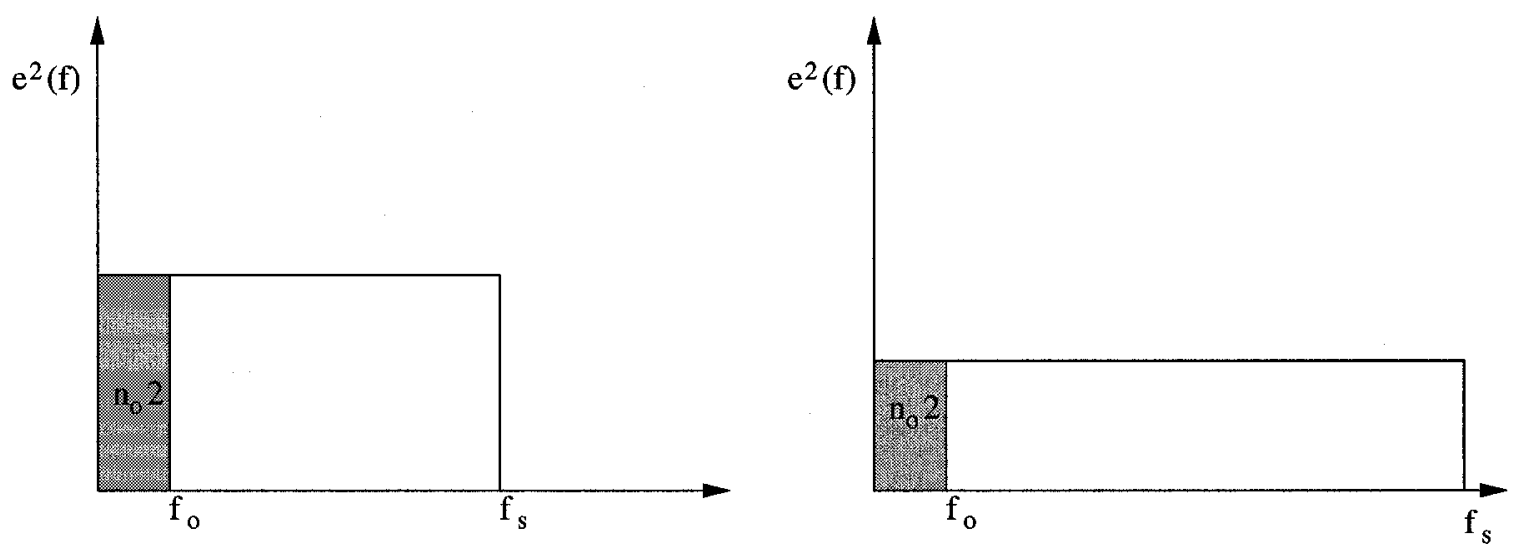

Figure 2.10: In-band Noise Decreases with Increasing Sampling Frequency

\subsubsection{Noise-Shaping}

Noise Shaping distributes noise energy unevenly over a frequency range. It is a technique that also can be used to shift noise to higher frequencies, outside of the band of interest.

First-order noise shaping in the frequency domain is equivalent to time domain differentiation of the quantization noise:

$$
\begin{gathered}
n_{i}=e_{i}-e_{i-1} \\
N(f)=E(f)\left|1-e^{\frac{-j 2 \pi f}{f_{s}}}\right|=2 e_{r m s} \sqrt{\frac{2}{f_{s}}} \sin \left(\frac{\pi f}{f_{s}}\right)
\end{gathered}
$$


The spectral density, before and after shaping, is shown in Fig.2.11. Noise within the band of interest is shifted to higher frequencies.

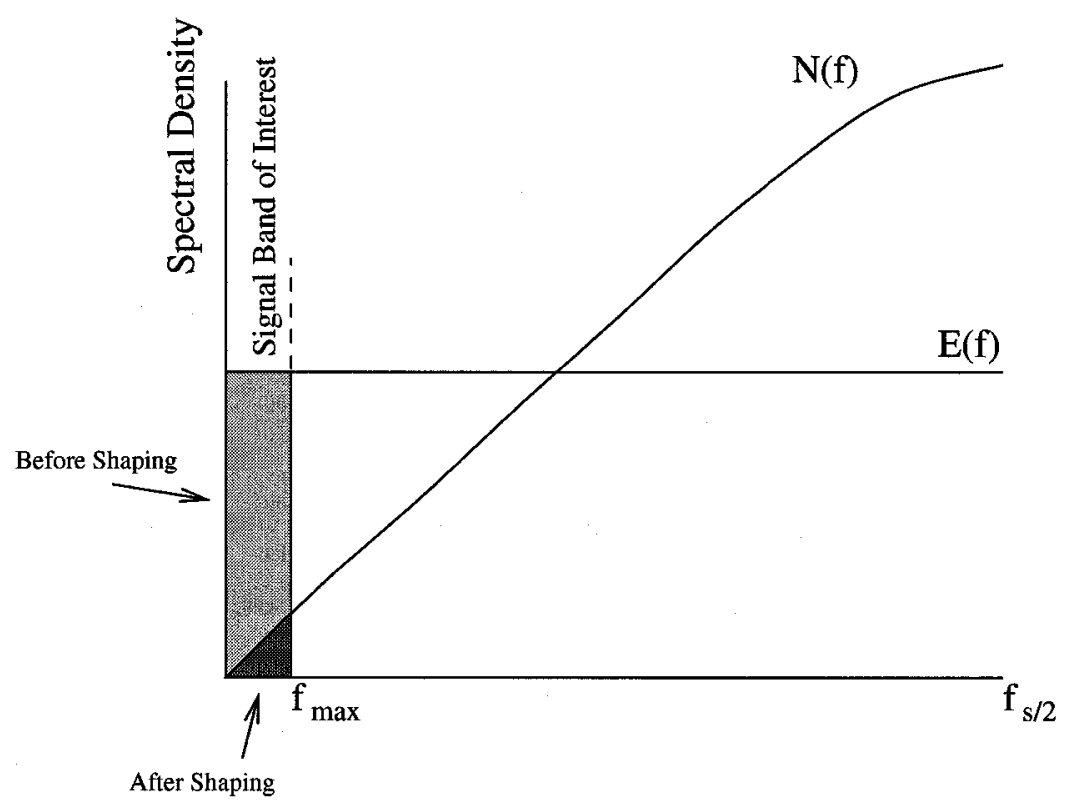

Figure 2.11: Noise Spectral Density before and after Shaping 


\subsection{First-Order $\Delta \Sigma$ Modulators}

The block diagram for a typical first-order $\Delta \Sigma$ Modulator is shown in Fig 2.7, and the corresponding linear model in Fig 2.12 [NST96].

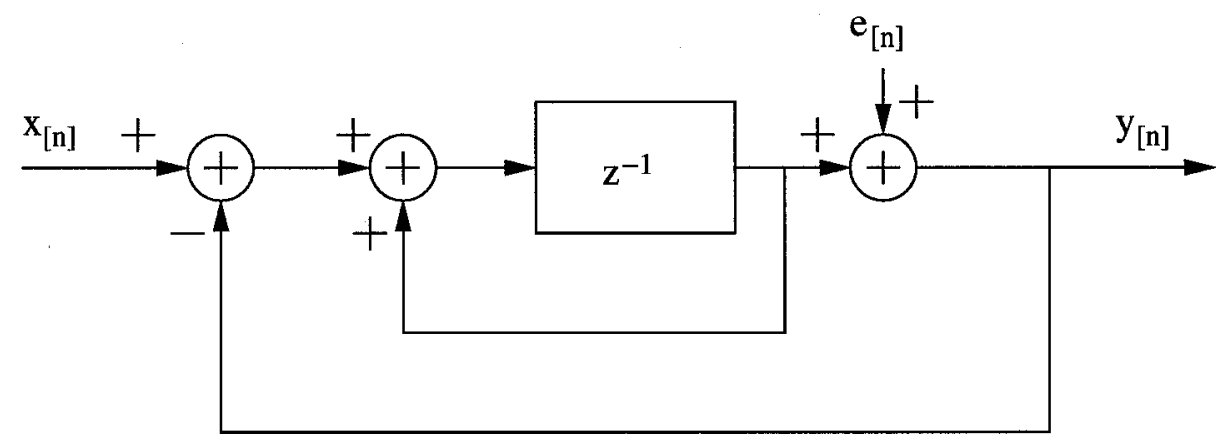

Figure 2.12: Linear Model of a First Order DSM

The addition of a quantizer to such a system makes it non-linear and its analytical solution very difficult if not impossible to obtain. In fact, to fully characterize the system in analytical form, one must make use of chaos theory.

The behaviour of the system can, however, be predicted using time-domain simulation methods.

The frequency domain linear model can provide us with some important system characteristics and usually can also provide adequate performance estimates for a wide variety of DSM architectures. In this thesis, both time-domain simulation and frequency domain linear approximation are used.

The output of the linear model (Fig.2.12) can be written:

$$
y[n]=x[n-1]+e[n]-e[n-1]
$$

The frequency-domain equivalent of this is:

$$
Y(z)=z^{-1} X(z)+E(z)\left(1-z^{-1}\right)
$$


In the frequency domain, the output is the sum of an undistorted input and the first-order shaped quantization noise.

The total noise power in the band of interest is given by:

$$
n_{o}^{2}=\int_{0}^{f_{o}}|N(f)|^{2} d f=e_{r m s}^{2} \frac{\pi^{2}}{3}\left(2 \frac{f_{o}}{f_{s}}\right)^{3}, \quad f_{s}^{2} \gg f_{o}^{2}
$$

In this case, it is seen that each doubling of the sampling frequency $f_{s}$ reduces the in-band noise power to one eighth $(9 \mathrm{~dB})$ of the original value. 


\subsection{Second-Order $\Delta \Sigma$ Modulators}

The in-band noise can be further reduced by increasing DSM order.

There are two ways to achieve higher order DSMs, namely Multi Stage Noise Shaping (MASH), and single-loop multi-order. These methods differ only at the circuit implementation level. They have identical system transfer functions which can be derived from the linear model. The pros and cons of these two approaches are described in [NST96].

A typical single-loop, second-order, DSM is shown in Fig.2.13.

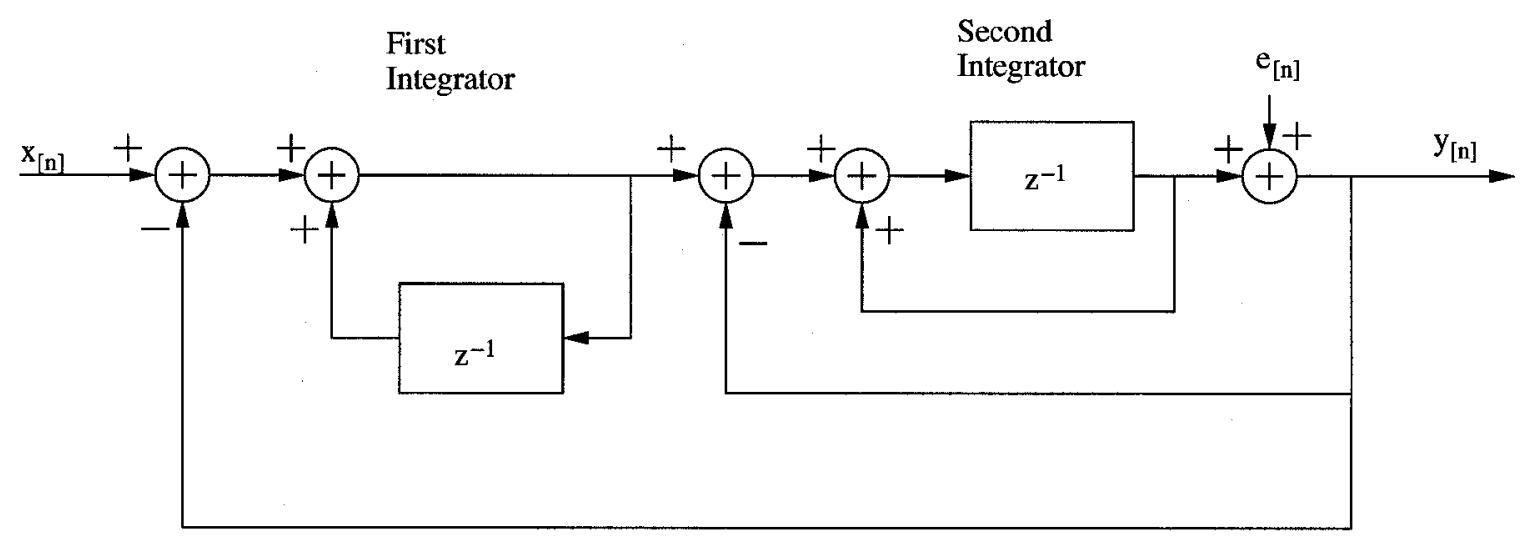

Figure 2.13: Linear Model of a Second Order DSM

The output of this modulator can be written as:

$$
y[n]=x[n-1]+(e[n]-2 e[n-1]+e[n-2])
$$

where the quantization noise is twice differentiated. The spectral density of the noise is given by:

$$
N(f)=E(f)\left(1-e^{-j 2 \pi \frac{f}{f_{s}}}\right)^{2}
$$

The in-band noise power is given by

$$
n_{o}^{2}=\int_{0}^{f_{o}}|N(f)|^{2} d f=e_{r m s}^{2} \frac{\pi^{4}}{5}\left(2 \frac{f_{o}}{f_{s}}\right)^{5} \quad f_{s}^{2} \gg f_{o}^{2}
$$


In this second-order case, the noise decreases by a factor of $32(15 \mathrm{~dB}$ ) for every doubling of $f_{s}$, compared to the factor of $8(9 \mathrm{~dB})$ which was obtained with the previouslydiscussed first-order DSM. 


\subsection{Higher-Order $\Delta \Sigma$ Modulators}

Higher-order $\Delta \Sigma$ Modulators for even better in-band noise reduction are possible. In general, for an L-order system, the noise spectral density and power figures are:

$$
\begin{gathered}
N_{L}(f)=E(f)\left(1-e^{-j 2 \pi \frac{f}{f_{s}}}\right)^{L} \\
n_{o_{L}}^{2}=\int_{0}^{f_{o}}|N(f)|^{2} d f=e_{r m s}^{2} \frac{\pi^{2 L}}{2 L+1}\left(2 \frac{f_{o}}{f_{s}}\right)^{2 L+1} f_{s}^{2} \gg f_{o}^{2}
\end{gathered}
$$

In the order-L case, the noise decreases by $3(2 L+1) \mathrm{dB}$ for every doubling of the sampling frequency. Tonal behaviour can be a problem in lower order modulators, especially first-order. Use of higher order significantly reduces this problem.

Although higher order DSMs can give superior performance, their use is discouraged by the difficulties in implementing circuits containing more than two integrators. Detailed discussions of the problem can be found in [NST96] and [CT92]. 


\section{Chapter 3}

\section{Analysis of a Second-Order $\Delta \Sigma$ FD Having a Fractional-N Divider and a Multi-Bit Quantizer}

In this chapter, the architecture of the new $\Delta \Sigma$ FD will be developed from a first-order $\Delta \Sigma$ FD design. Further refinements to this design result in the final device. The improvements on the starting first-order $\Delta \Sigma$ FD are:

- Second-order $\Delta \Sigma$ FD with integer-N divider and single-bit quantizer.

The slope of the noise shaping is increased from $20 \mathrm{~dB} / \mathrm{dec}$ in the first-order circuit to $40 \mathrm{~dB} / \mathrm{dec}$ in the second-order one. The increase in the amount of quantization noise power shifted to higher frequencies results in a higher in-band SNR.

- Second-order $\Delta \Sigma$ FD with fractional-N divider and single-bit quantizer.

The use of a fractional-N divider to reduce total quantization noise power will also bring about improved in-band SNR. Total quantization noise power is directly related 
to the quantizer's minimum step size. The replacement of the integer- $\mathrm{N}$ divider with a fractional- $\mathrm{N}$ divider enables one to use smaller minimum quantizer step sizes. A $\frac{1}{8}$ fractional-N divider is used for the work described in this thesis, so that the minimum step size is reduced to $\frac{1}{8} t h$ of that of the original, and the total noise power is reduced to $\frac{1}{64}$ th of the original.

- Second-order $\Delta \Sigma \mathrm{FD}$ with fractional-N divider and multi-bit quantizer (the proposed architecture).

While a fractional-N divider reduces quantization noise and increases in-band SNR and frequency resolution, it does have a serious drawback: the input frequency range is reduced to $\frac{1}{8} t h$ of the original one. This reduction in frequency range can be compensated by using a multi-bit quantizer (three bits in the present case). 


\subsection{First-Order $\Delta \Sigma$ FD}

The block diagram for a first-order $\Delta \Sigma$ FD is shown in Fig.3.1 and its timing diagram is shown in Fig.3.2.

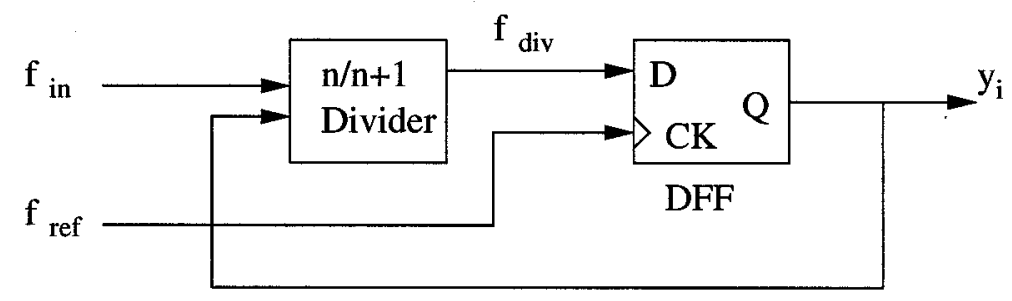

Figure 3.1: Block Diagram for a First-Order $\Delta \Sigma$ FD

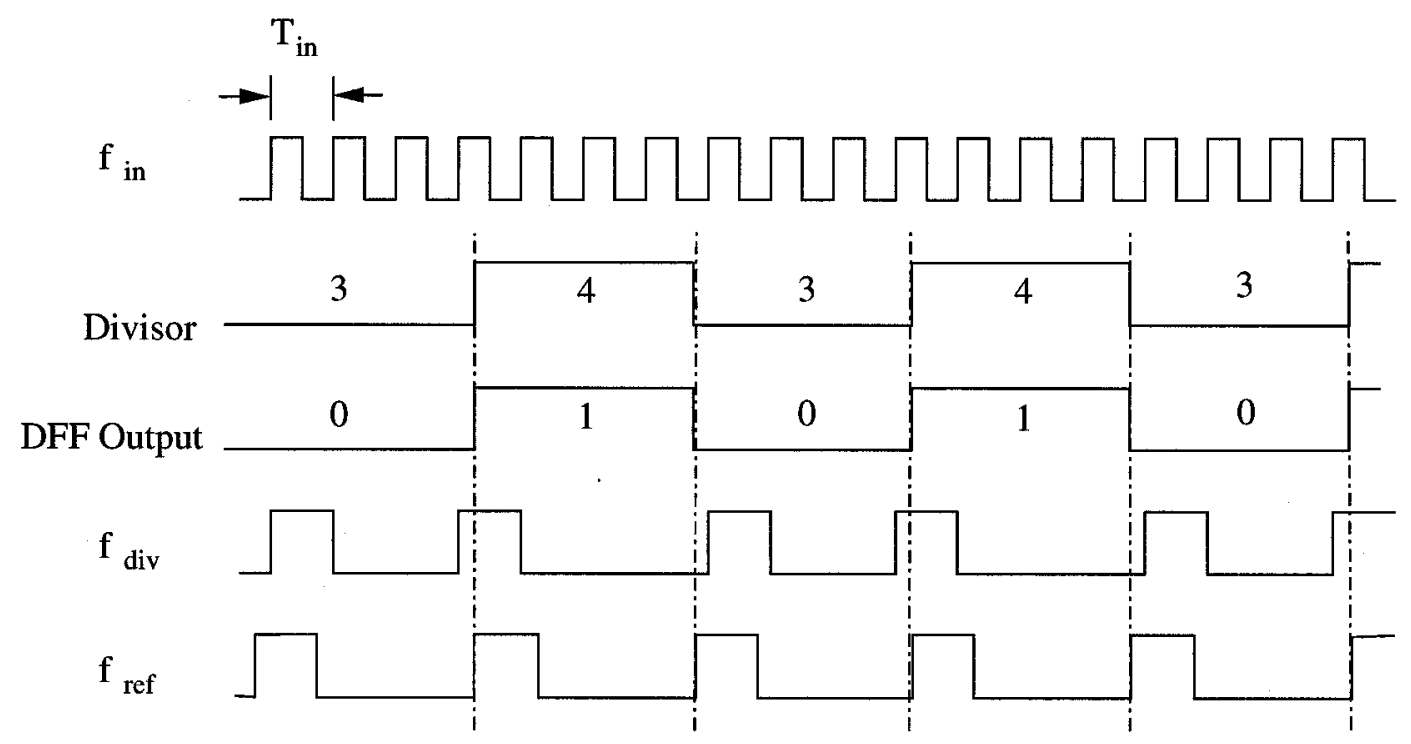

Figure 3.2: Timing Diagram for a First-Order $\Delta \Sigma$ FD

In the block diagram shown in Fig.3.1, the divisor for the next $f_{\text {ref }}$ cycle is controlled by the output of a D-type Flip Flop (DFF) functioning as a phase comparator: when the rising edge of the divided input signal $f_{d i v}$ leads that of the reference signal $f_{r e f}$, the DFF 
output is high. This will cause the input signal, $f_{\text {in }}$, to be divided by $n+1$ during the next cycle, and the rising edge of $f_{d i v}$ to be delayed by one $f_{\text {in }}$ period, $T_{i n}$. Eventually, $f_{d i v}$ will come to lag $f_{r e f}$ and the DFF output will go low. The input signal $f_{i n}$ will once again be divided by $n$, a situation that will persist until $f_{d i v}$ eventually comes to lead $f_{\text {in }}$ again.

For the system to work, $f_{\text {in }}$ must satisfy:

$$
n f_{r e f}<f_{\text {in }}<(n+1) f_{r e f}
$$

If $f_{\text {in }}$ is less than $n f_{r e f}$, the DFF output will always be low. If $f_{\text {in }}$ is greater than $(n+$ 1) $f_{r e f}$, the output will be permanently high. These frequency restrictions of the $\Delta \Sigma$ FD are analogous to the voltage amplitude constraints to which a conventional $\Delta \Sigma$ modulator is subject to.

The first-order $\Delta \Sigma$ FD 's output bit-stream corresponds to the instantaneous input frequency plus quantization noise. 


\subsubsection{Linear Model for Dual-Modulus Divider}

Linearization of the dual modulus divider is the key to analyzing the behaviour of the first order $\Delta \Sigma$ FD. Phase information can be extracted from the timing diagram shown in Fig.3.2. An example of phase ramps for $f_{r e f}$ and $f_{d i v}$ is illustrated in Fig.3.3.

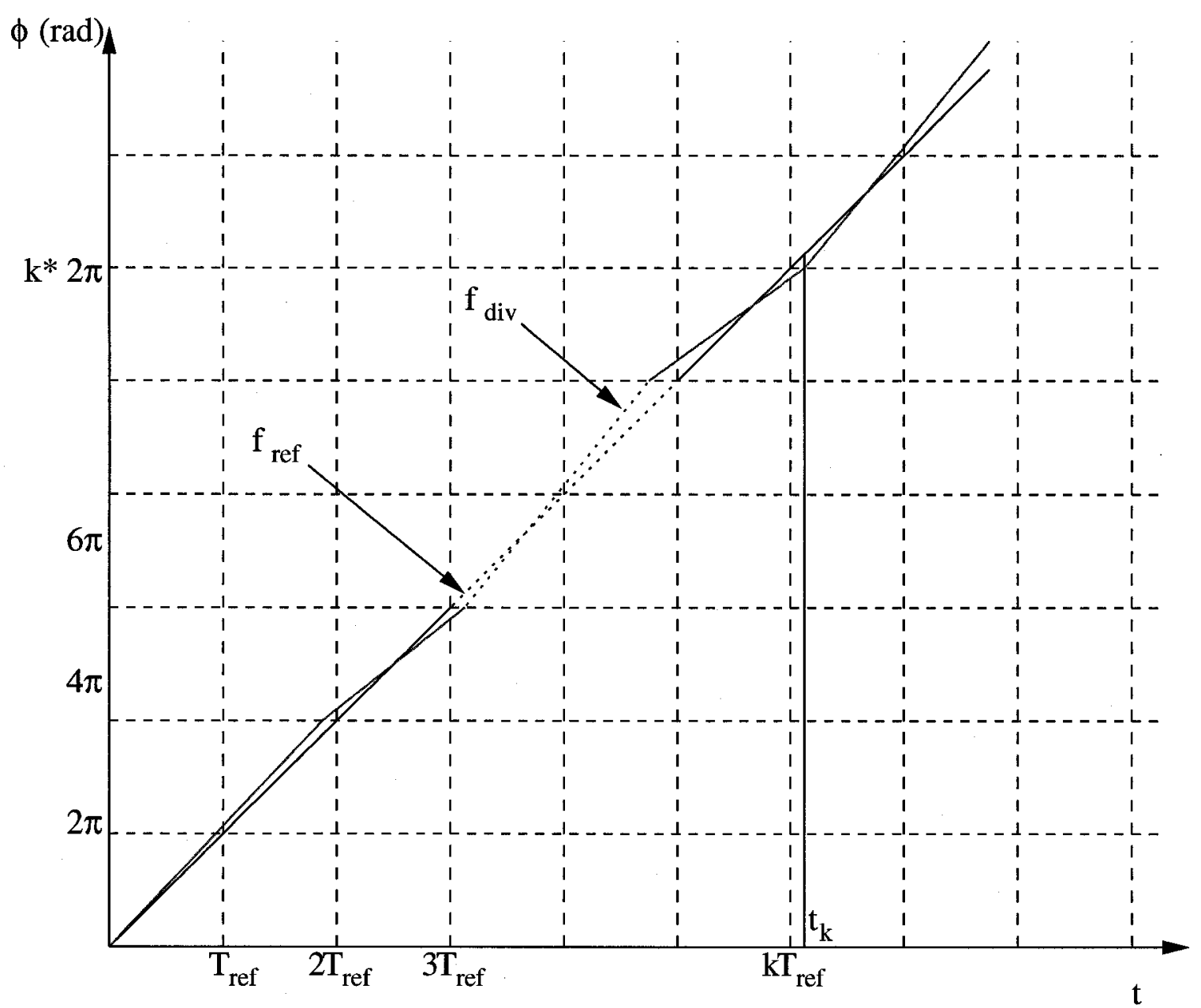

Figure 3.3: Phase Ramps for $f_{r e f}$ and $f_{d i v}$

Where, $t_{k}$ denotes the end of the $k_{t h}$ divider cycle. 
The phase of $f_{d i v}$ with respect to $f_{r e f}$ is given by:

$$
\begin{gathered}
\phi_{f_{\text {div }}}=2 \pi \frac{t_{k}}{T_{r e f}} \\
t_{k}=\sum_{i=1}^{k}\left(n+b_{i}\right) T_{\text {in }}(k),
\end{gathered}
$$

Where $T_{i n}(k)$ is the average period of the input frequency during the $k_{t h}$ divider cycle, $n$ is the base divisor (At any instant of time, the divider is dividing by $n$ or by $n-1$ ), and $b_{i}$ may be either ' 0 ' or ' 1 '.

The average frequency is

$$
f_{\text {in }}(k)=\frac{1}{T_{\text {in }}(k)}=f_{\text {nom }}+f_{\text {mod }}(k),
$$

Where $f_{\text {nom }}$ is the nominal carrier frequency and $f_{\text {mod }}(k)$ the average modulating frequency added to $f_{\text {nom }}$ during the $k_{t h}$ divider cycle.

The nominal divisor is the ratio

$$
N=\frac{f_{\text {nom }}}{f_{\text {ref }}},
$$

Substitution of $\mathrm{Eq}(3.3), \mathrm{Eq}(3.4)$ and $\mathrm{Eq}(3.5)$ into $\mathrm{Eq}(3.2)$ gives

$$
\begin{aligned}
\phi_{f_{r e f}} & =2 \pi \frac{\sum_{i=1}^{k}\left(n+b_{i}\right) \frac{1}{f_{i n(k)}}}{T_{r e f}} \\
& =2 \pi \sum_{i=1}^{k}\left[\frac{1}{N}\left(n+b_{i}\right) \frac{f_{n o m}}{f_{i n}(k)}\right]
\end{aligned}
$$

A block diagram for the linearized circuit model is shown in Fig.3.4. 


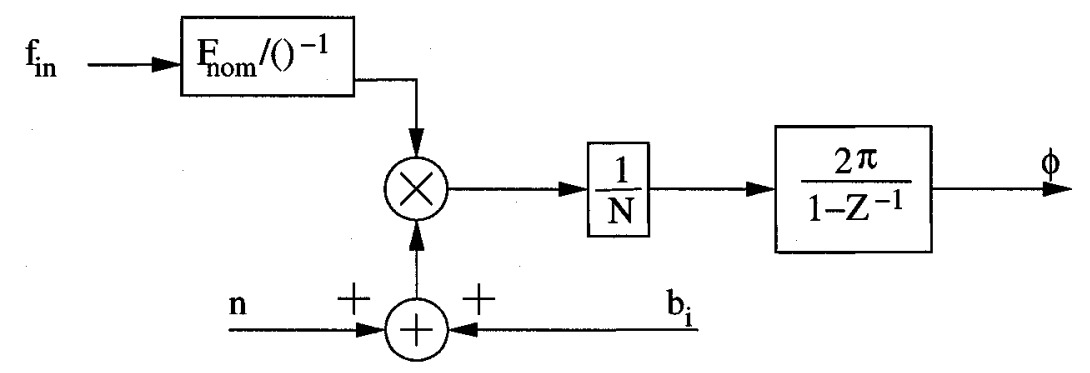

Figure 3.4: Linear Model for Dual-Modulus Divider

\subsubsection{Linear Model for a First Order $\Delta \Sigma$ FD}

The complete model for a first-order $\Delta \Sigma$ FD can now be obtained through the inclusion of a divider, a phase detector and a one-bit quantizer.

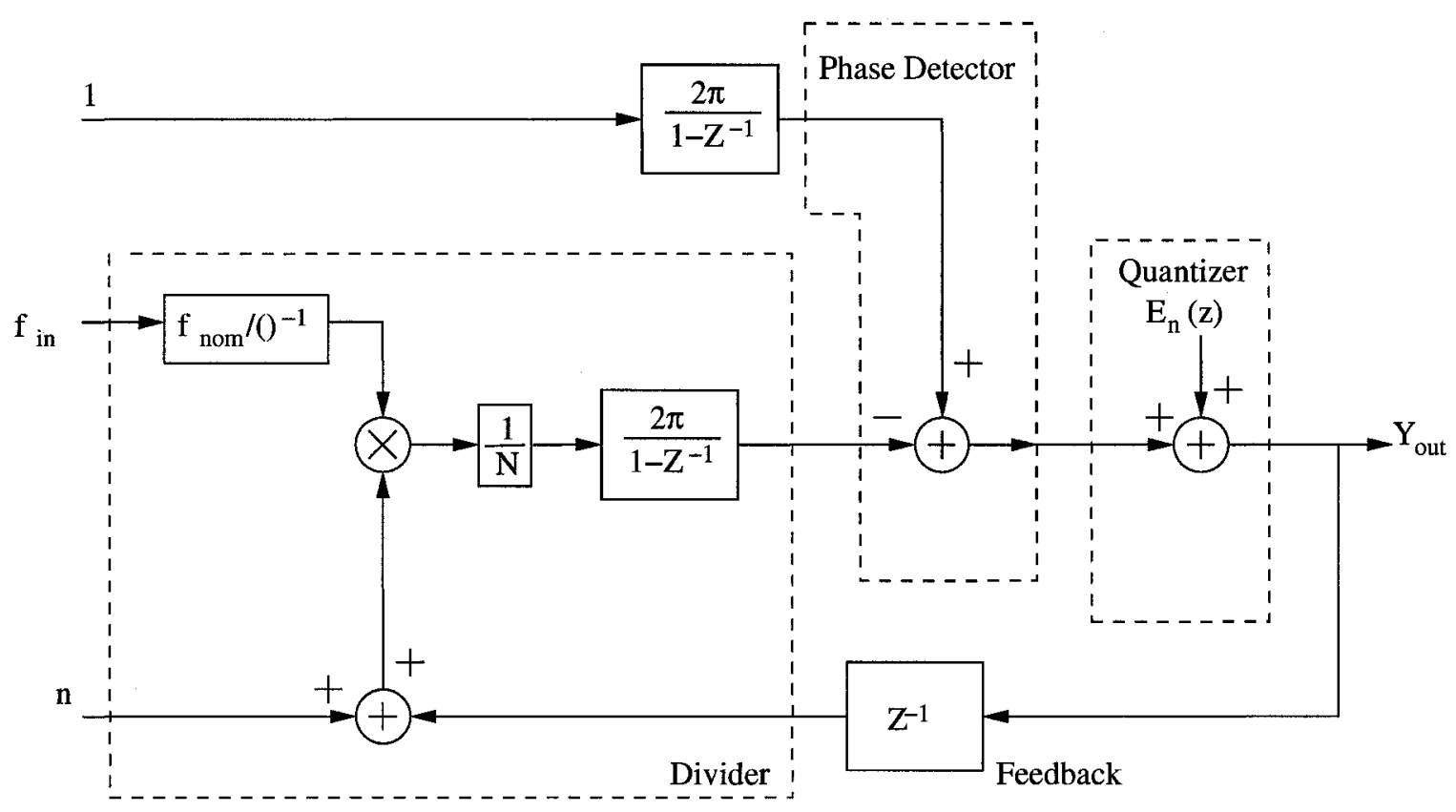

Figure 3.5: Linear Model for a First-Order $\Delta \Sigma$ FD

If the input instantaneous frequency is given as in Eq.3.7 (a special case of the generic 
form in Eq.2.1).

$$
f_{\text {in }}=f_{\text {nom }}+\Delta f \sin (\Omega t)
$$

Where $f_{\text {nom }}$ is the nominal carrier frequency, $\Delta f$ the maximum frequency deviation, and $\Omega$ the modulating frequency.

The output of the system in Fig.3.5 is

$$
Y_{\text {out }}=N-n+\frac{\Delta f}{f_{\text {ref }}} \sin (\Omega t+\theta)+\left(1-z^{-1}\right) E_{n}
$$

Where $N=\frac{f_{\text {nom }}}{f_{\text {ref }}}$, the ratio of nominal carrier frequency to reference frequency, $n$ is the base divisor and $E_{n}$ is the quantization noise spectral density. The output contains a DC offset $(N-n)$ proportional to the center frequency offset, an undistorted and scaled version of the modulation signal, and first-order differentiated quantization noise.

\subsubsection{Performance Evaluation}

When the input frequency to a first-order $\Delta \Sigma \mathrm{FD}$ is constant, the quantization noise will not be white, as previously assumed in Chapter 2 . In this case, the linear model fails to describe the operation of the system accurately.

To sidestep this problem and evaluate the performance, a sinusoidally-modulated input frequency (Eq.3.7) is used.

Signal-To-Noise Ratio(SNR), the ratio of Signal Power to Noise Power within a prescribed band, is the performance metric of interest.

If $f_{\text {low }}$ and $f_{\text {high }}$ are the band boundaries, the total in-band noise power is:

$$
S_{\text {noise }}=E_{n}^{2} \int_{f_{\text {low }}}^{f_{\text {high }}}\left|1-e^{-j \frac{2 \pi f}{f_{\text {ref }}}}\right|^{2} d f
$$


Where

$$
\begin{gathered}
E_{n}^{2}=\frac{\Delta^{2}}{12 f_{s}} \\
\Delta=f_{r e f} \\
f_{s}=f_{r e f}
\end{gathered}
$$

The signal power is given by

$$
S_{\text {sig }}=\frac{\Delta f^{2}}{2}
$$

Where $\Delta f$ is the maximum frequency deviation, as expressed in Eq.3.7.

In order to compare analytical results with simulation results, numbers are substituted into the equations.

$\Delta f$, the maximum frequency deviation, has to be $<\frac{f_{\text {ref }}}{2}$ in order for the system not to saturate, as described in Eq.3.1.

Because the reference clock, $f_{r e f}$, is also the sampling frequency in the system, $\Omega$, the modulating frequency, has to be $<\frac{f_{\text {ref }}}{2}$ to satisfy Nyquist's Law.

Let $f_{\text {ref }}=10 \mathrm{MHz}, \Delta f=-10 \mathrm{~dB}$ of full range $\frac{f_{\text {ref }}}{2}, f_{\text {low }}=10 \mathrm{kHz}, f_{\text {high }}=200 \mathrm{kHz}$, and $\Omega=50 \mathrm{kHz}$

The SNR predicted by theory ( Eq.3.9) is compared to that computed by simulation shown in Fig.3.6 over the bandwidth from $f_{\text {low }}=10 \mathrm{kHz}$ to $f_{\text {high }}=200 \mathrm{kHz}$.

The equation predicts that $S N R$ in the band will be $31.54 \mathrm{~dB}$.

The Matlab simulation result finds the $S N R$ to be $31.7 d B$ using Eq.3.14.

$$
S N R=\frac{\sum(\text { SignalPowerat } 50 \mathrm{kHzFFT \text {bin } )}}{\sum(\text { FFTbinsfrom } 10 \mathrm{kHz} \text { to } 200 \mathrm{kHz})-\sum(\text { SignalPowerat } 50 \mathrm{kHzFFTbin})}
$$




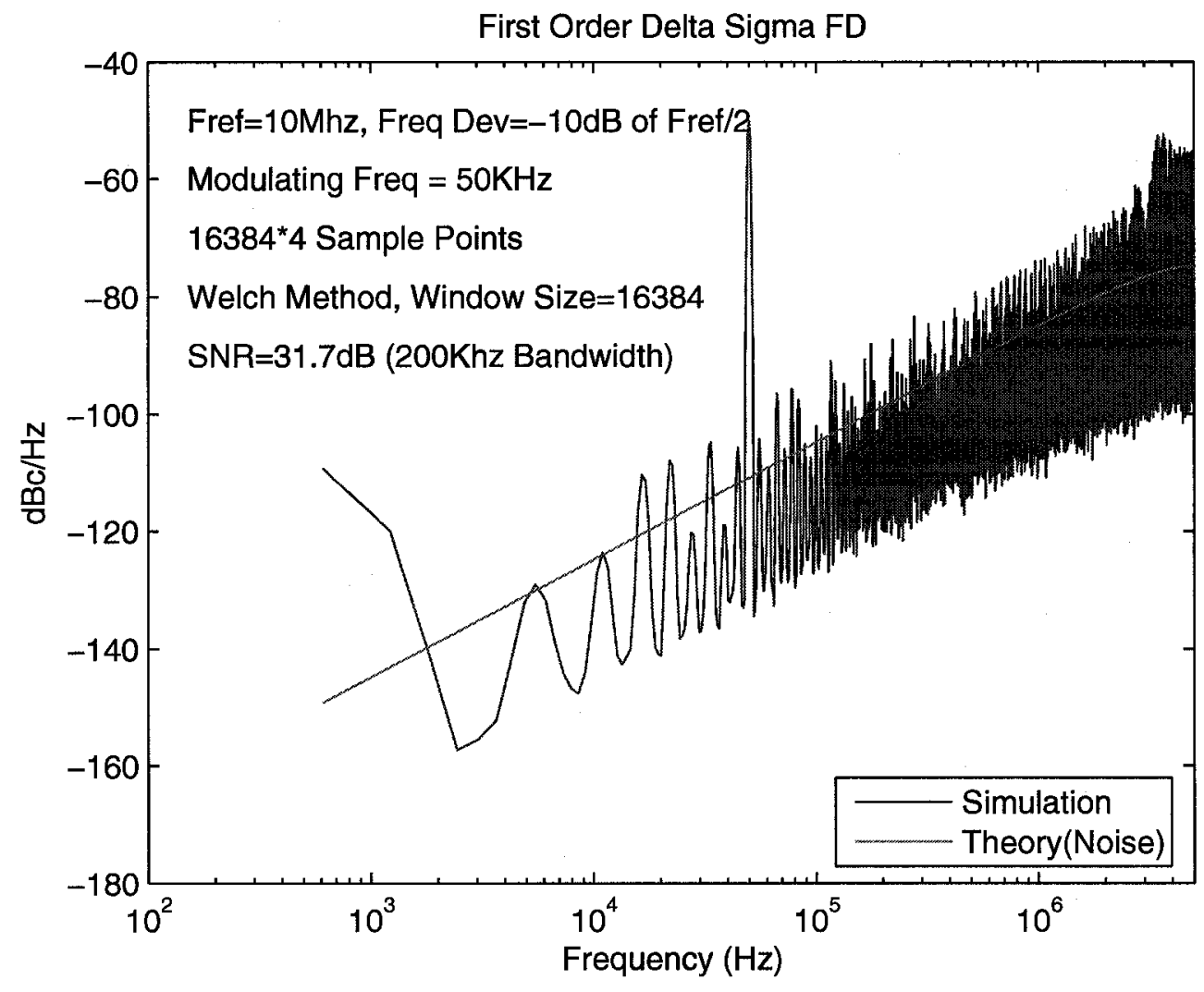

Figure 3.6: Simulation of First Order $\Delta \Sigma$ FD

This is a rather good agreement, and the SNR calculated by simulation will henceforth be used as a benchmark. 


\subsection{Second-Order $\Delta \Sigma$ FD with Integer-N Divider and Single- Bit Quantizer}

The effects on in-band noise power due to moving to a $2^{\text {nd }}$ order system are now examined. The block diagram and the corresponding linearized model for such a $\Delta \Sigma$ FD are depicted in Fig.3.7 and Fig.3.8:

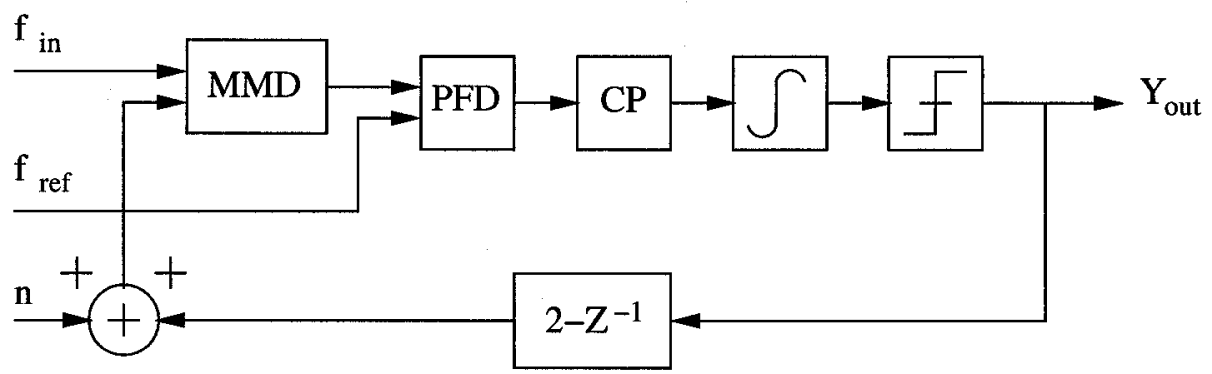

Figure 3.7: Second Order $\Delta \Sigma$ FD Block Diagram
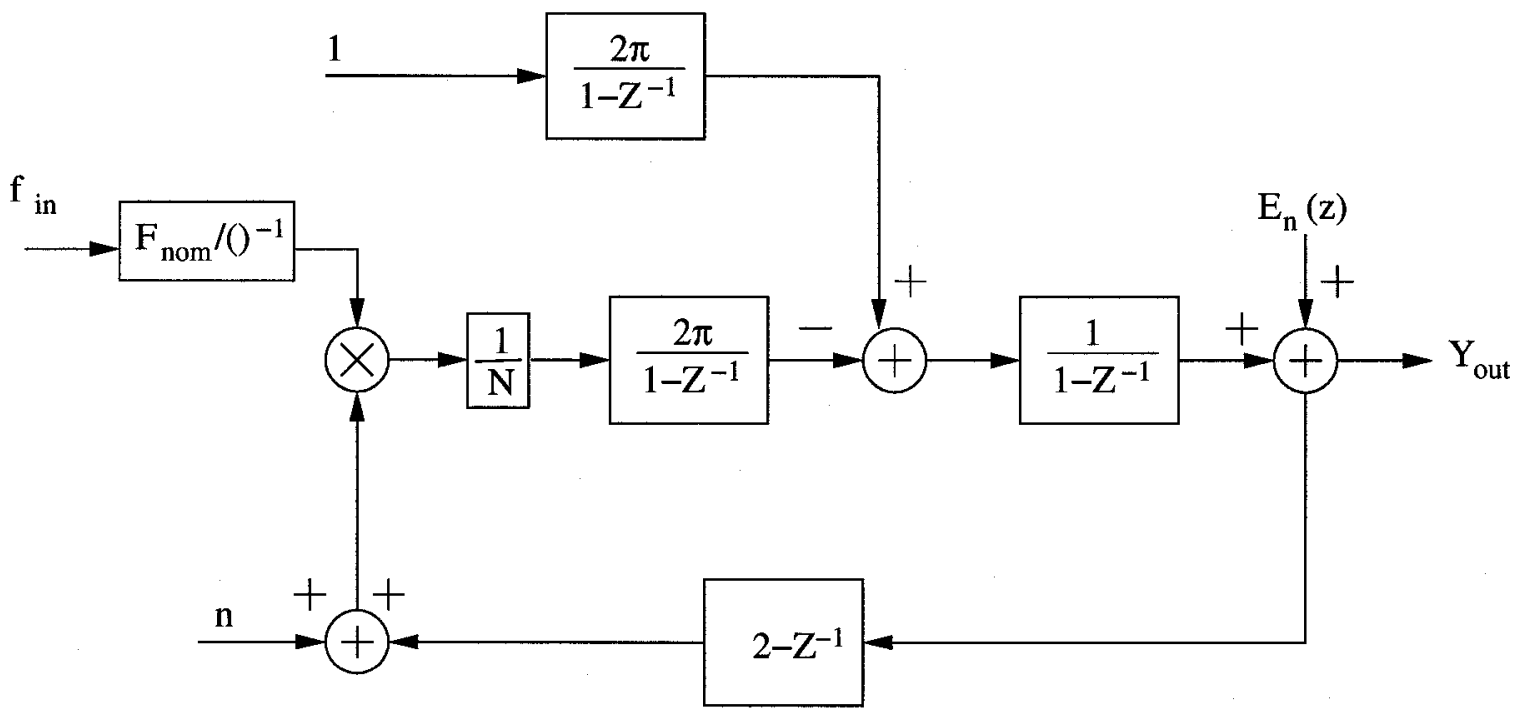

Figure 3.8: Second Order $\Delta \Sigma$ FD Linear Model 
The output of the system is given by:

$$
Y_{\text {out }}=N-n+\frac{\Delta f}{f_{\text {ref }}} \sin (\Omega t)+\left(1-z^{-1}\right)^{2} E_{n},
$$

and the system noise power is

$$
S_{\text {noise }}=E_{n}^{2} \int_{f_{\text {low }}}^{f_{\text {high }}}\left|1-e^{-j \frac{2 \pi f}{f_{\text {ref }}}}\right|^{4} d f
$$

The quantization noise, as shown in Eq.3.15, is second-order differentiated, so that more noise will be shifted upwards in frequency and out of the band of interest. 
The theoretical performance of the second order system was compared to simulation results and the SNR calculated with $E_{n}^{2}, \Delta$ and $f_{s}$ retaining their values from the first-order example.

The Matlab simulation result is shown in Fig.3.9.

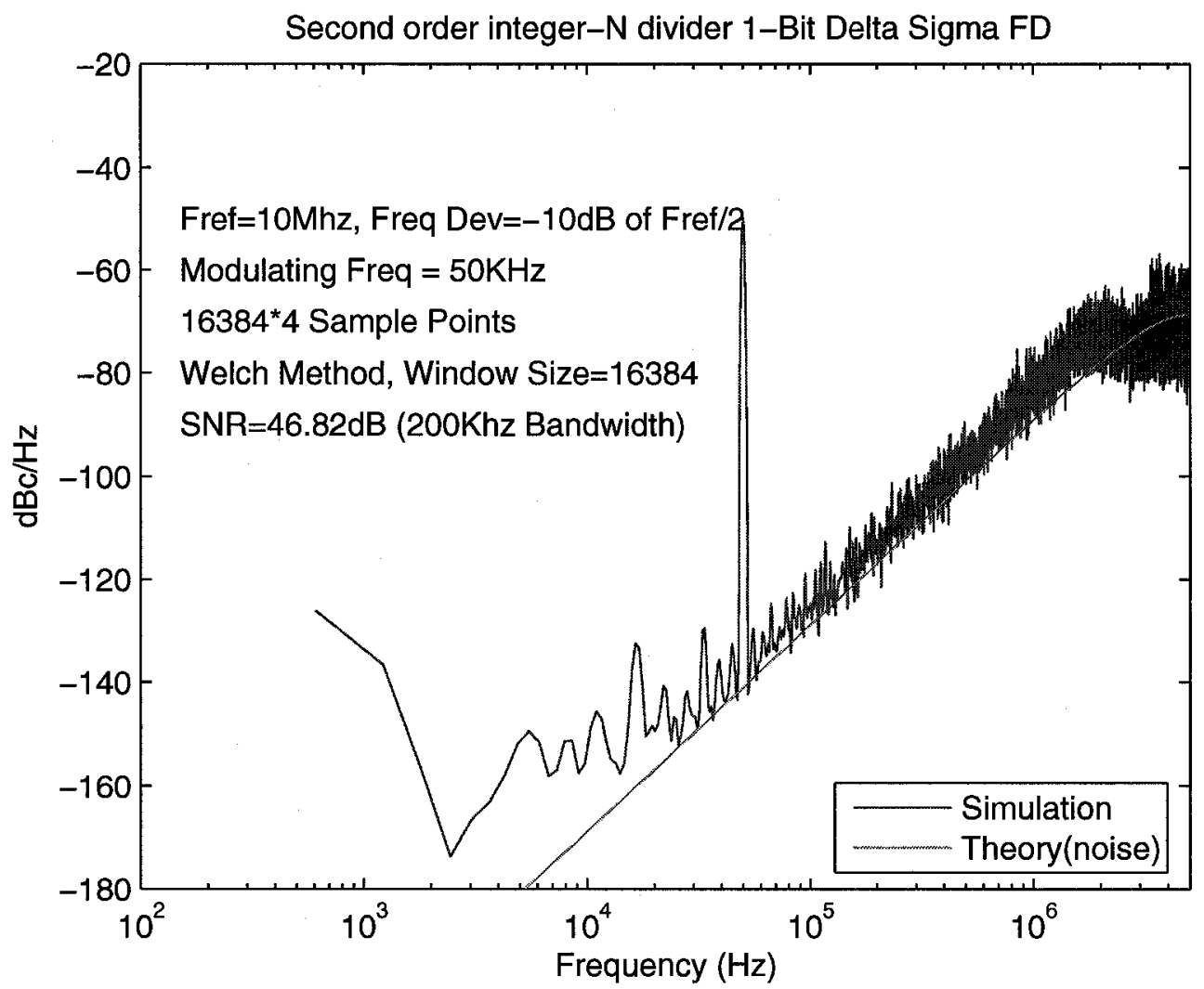

Figure 3.9: Matlab Simulation Result for the Second Order $\Delta \Sigma$ FD

The theoretically-predicted $S N R$ was $51.73 \mathrm{~dB}$, a figure nearly $20 \mathrm{~dB}$ greater than that of the first-order $\Delta \Sigma$ FD. The difference can be attributed to decreased in-band quantization noise. 
The $S N R$ found by simulation was about $4 \mathrm{~dB}$ lower than the theoretical result. The main reason for the difference is the linear model's inability to account for the non-linear nature of the system. 


\subsection{Second-Order FD with Fractional-N Divider}

Further reduction in overall quantization noise power may be had if the quantization step size is reduced.

In the systems described previously, the divisor is restricted to integer values ( $n$ and $n+1$ ), and the minimum quantization step size is correspondingly restricted. If, however, a non-integer divisor was available, finer control over this step size could be achieved and the quantization noise power would be reduced to a fraction of what it would be otherwise. This has been done, with the divider being implemented by an accumulator-based circuit.

In the past, this approach to fractional-N division has been beset by truncation-related discrete spurs [Cra94]. The many ways of suppressing these spurs proposed to date have all involved the use of difficult-to-implement precision analog circuits. One example is shown in Fig.3.10 [Roh83]. It is very difficult to create a precise match between the D/A output and phase error signal.

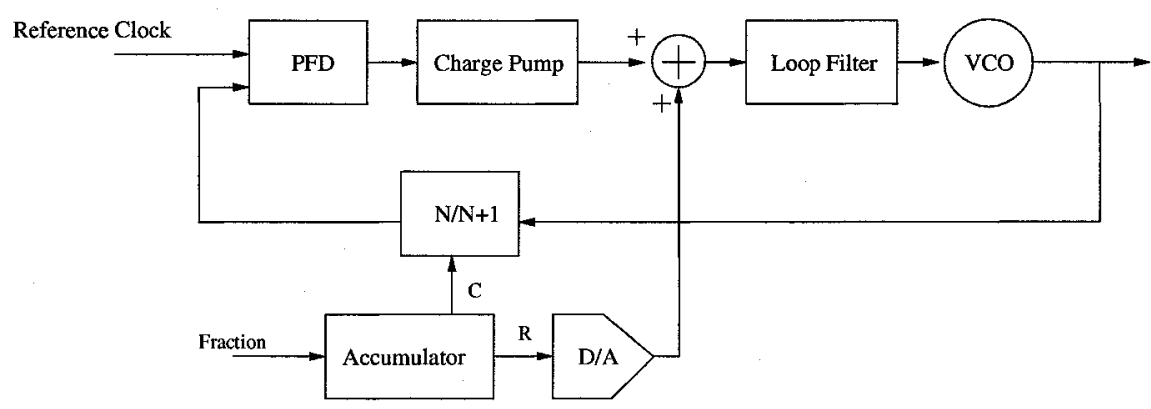

Figure 3.10: An Example of Phase Error Cancellation in Fractional-N Frequency Synthesis

A novel approach to implement a spur-free fractional-N divider is illustrated in Fig.3.11. The multi-modulus divider has two outputs: $f_{d_{0}}$ and $f_{d_{1}}, f_{d_{0}}$ is one $T_{i n}$ ahead of $f_{d_{1}}$. These two signals, along with the Selector and the Phase Frequency Detector (PFD)/Charge Pump (CP) array, form a D/A for cancelling out the truncation error from the accumulator. 
Fref

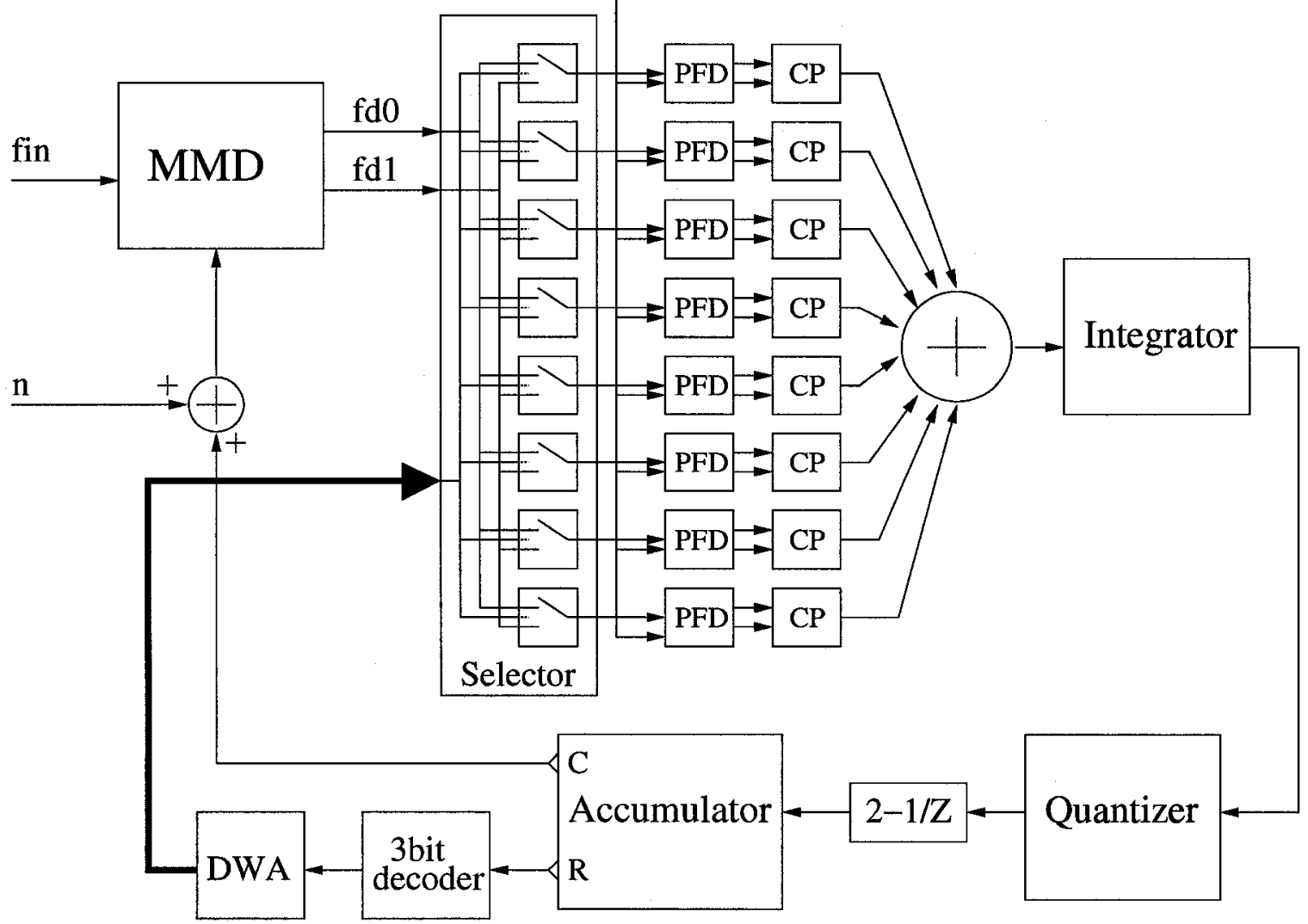

Figure 3.11: Block Diagram for a Second-Order $\Delta \Sigma$ FD With Fractional-N Divider

Other architectures incorporating a separate D/A in the feedback path focus on techniques to match the D/A output charge with the charge transferred through the PFD and CP. In the architecture shown in Fig.3.11, the components making up the D/A are actually the Selector, the PFDs and CPs. Therefore no separate D/A is needed and no mismatch between the $\mathrm{D} / \mathrm{A}$ and the charge pump exists. The mismatch errors that cannot be eliminated (except through careful layout techniques) include those due to $f_{d_{1}}$ not being delayed by exactly one $T_{\text {in }}$ cycle, delay mismatches in the selector, and mismatches in the PFD/CP 
elements. This approach anticipates advantages of applying dynamic element matching techniques to those mismatches.

A linear model for the system is shown in Fig.3.12.

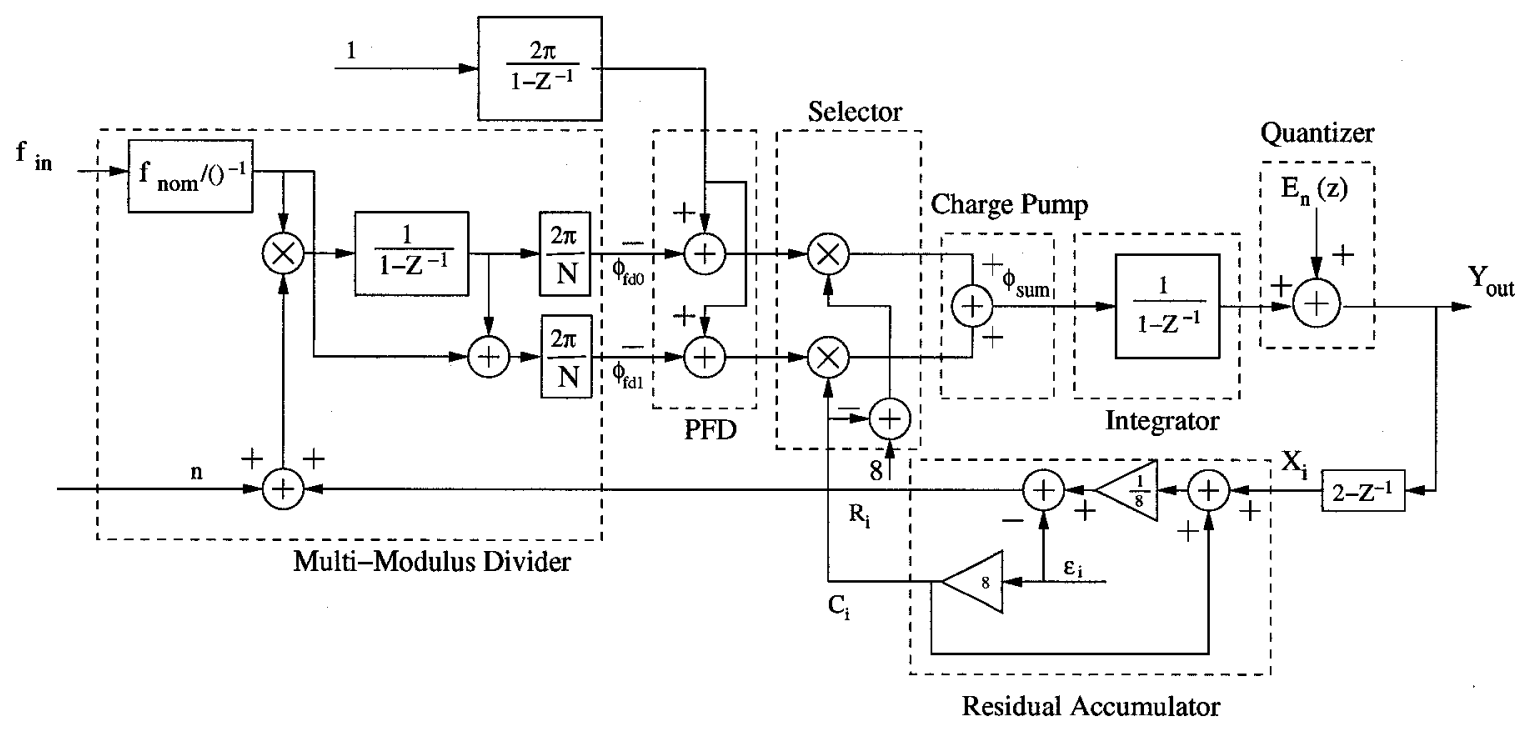

Figure 3.12: Linear Model of Second Order $\Delta \Sigma$ FD with Fractional-N Divider

Referring to the linear model, a proof that the accumulator truncation error $\left(\varepsilon_{i}\right)$ is cancelled and does not appear at the Charge Pump output proceeds as follows:

$$
\begin{gathered}
\phi_{f d 0}=\frac{2 \pi}{N} \frac{1}{1-Z^{-1}} \frac{f_{\text {nom }}}{f_{\text {in }}}\left(n+C_{i}\right) \\
\phi_{f d 1}=\frac{2 \pi}{N}\left(\frac{1}{1-Z^{-1}} \frac{f_{\text {nom }}}{f_{\text {in }}}\left(n+C_{i}\right)+\frac{f_{\text {nom }}}{f_{\text {in }}}\right) \\
C_{i}=\frac{X_{i}}{8}-\left(1-Z^{-1}\right) \varepsilon_{i} \\
R_{i}=8 \varepsilon_{i} \\
\phi_{\text {sum }}=\left(8-R_{i}\right)\left(\phi_{f_{\text {ref }}}-\phi_{f d 0}\right)+R_{i}\left(\phi_{f_{\text {ref }}}-\phi_{f d 0}\right),
\end{gathered}
$$


Where $X_{i}$ is the output of the $2-Z^{-1}$ block, $C_{i}$ and $R_{i}$ the accumulator carry and remainder outputs, respectively.

Some substitutions put Eq.3.21 into the form:

$$
\begin{aligned}
\phi_{\text {sum }}= & \frac{2 \pi}{1-Z^{-1}}\left\{8 \left[1-\frac{1}{N} \frac{f_{\text {nom }}}{f_{\text {in }}}\left[n+\frac{x_{i}}{8}+\left(1-Z^{-1}\right) \varepsilon_{i}\right]\right.\right. \\
& \left.-\frac{1}{N}\left(1-Z^{-1} 8 \varepsilon_{i}\right)\right\} \\
= & \frac{2 \pi}{1-Z^{-1}} 8\left\{1-\frac{1}{N} \frac{f_{\text {nom }}}{f_{\text {in }}}\left[n+\frac{x_{i}}{8}\right]\right\}
\end{aligned}
$$

$\phi_{\text {sum }}$ is independent of $\varepsilon_{i}$, as was to be shown.

The output of the system derived from the linear model is given by:

$$
Y_{\text {out }}=N-n+\frac{\Delta f}{f_{\text {ref }}} \sin (\Omega t+\theta)+\left(1-Z^{-1}\right)^{2} E_{n}
$$

This is the same expression as Eq.3.15, except that now the value of $E_{n}$ is $\frac{1}{8}$ of that in Eq.3.15.

This method of reducing noise power also causes the full scale frequency deviation $f_{\text {mod }}$ to be reduced to one eighth of the original, from $\pm \frac{f_{\text {ref }}}{2}$ to $\pm \frac{f_{\text {ref }}}{16}$. This drawback can be compensated through the use of a multi-bit quantizer, as will be shown in next section.

The results of simulating both types of second-order FDs are plotted in Fig.3.13. In each case, the same input was used: the modulating frequency was $\Omega=50 \mathrm{kHz}$ and frequency deviation $f_{\text {mod }}=-10 \mathrm{~dB}$ of $\frac{f_{\text {ref }}}{16}$

Analysis indicates that the SNR of an FD using a fractional-N divider is better than that obtained with an integer- $\mathrm{N}$ architecture by $20 \log _{10} 8=18 \mathrm{~dB}$, in agreement with timedomain simulation results. 

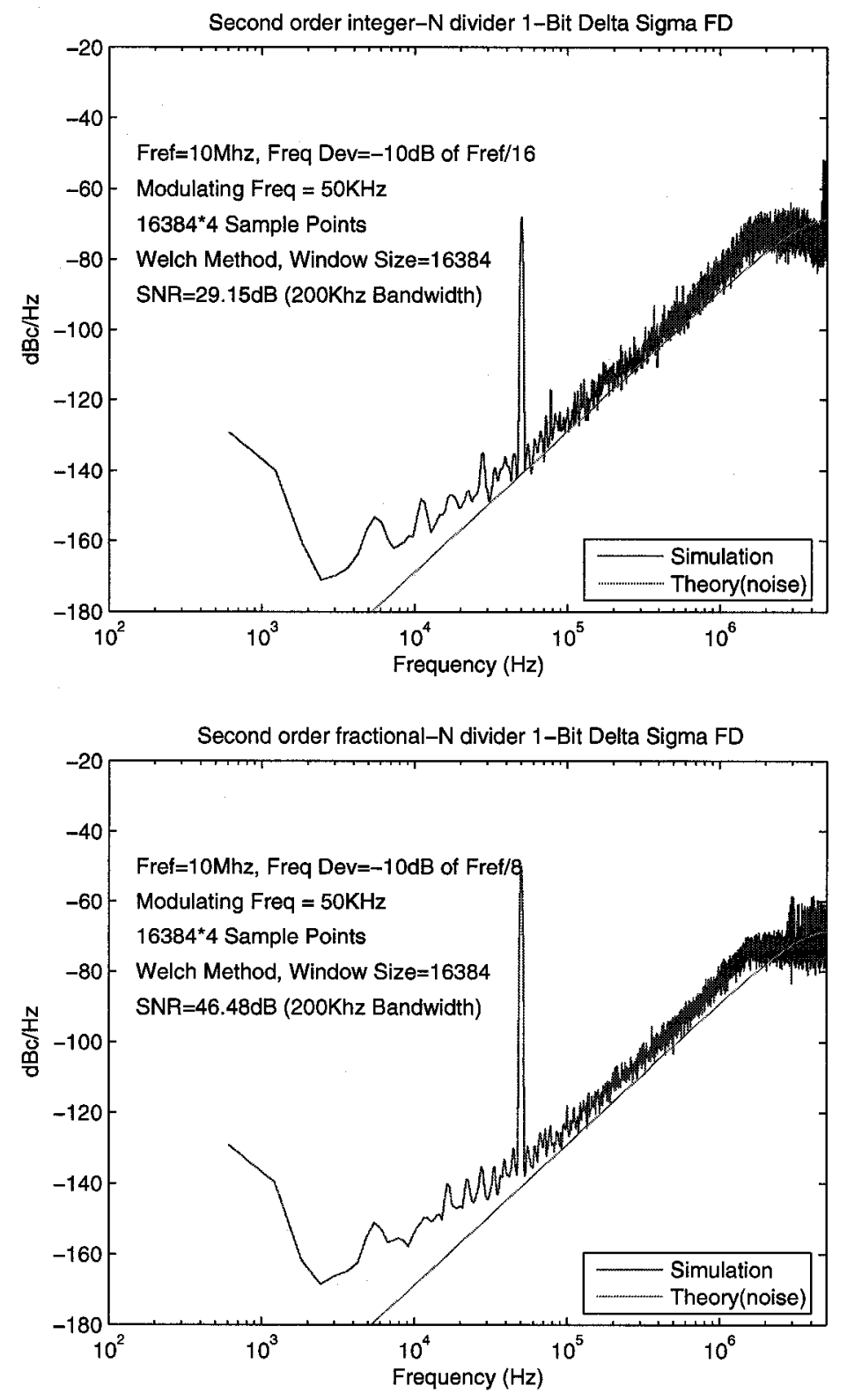

Figure 3.13: Integer-N versus Fractional-N Divider Comparison 


\subsection{A Second-Order $\Delta \Sigma$ FD Using a Fractional-N divider and a Three-Bit Quantizer (Proposed Architecture)}

As described in the previous section, the use of a fractional- $\mathrm{N}$ divider is accompanied by a reduced input frequency range. This drawback is overcome when the single-bit quantizer is replaced with a multi-bit quantizer. In particular, a 3-bit quantizer can restore the input frequency range to $\pm \frac{f_{\text {ref }}}{2}$. This is a feature of the proposed architecture. A block diagram is shown in Fig.3.14. This block diagram is identical to Fig.3.11, except that now the singlebit quantizer is replaced by a 3-bit quantizer. The corresponding linear model is that shown in Fig.3.15.

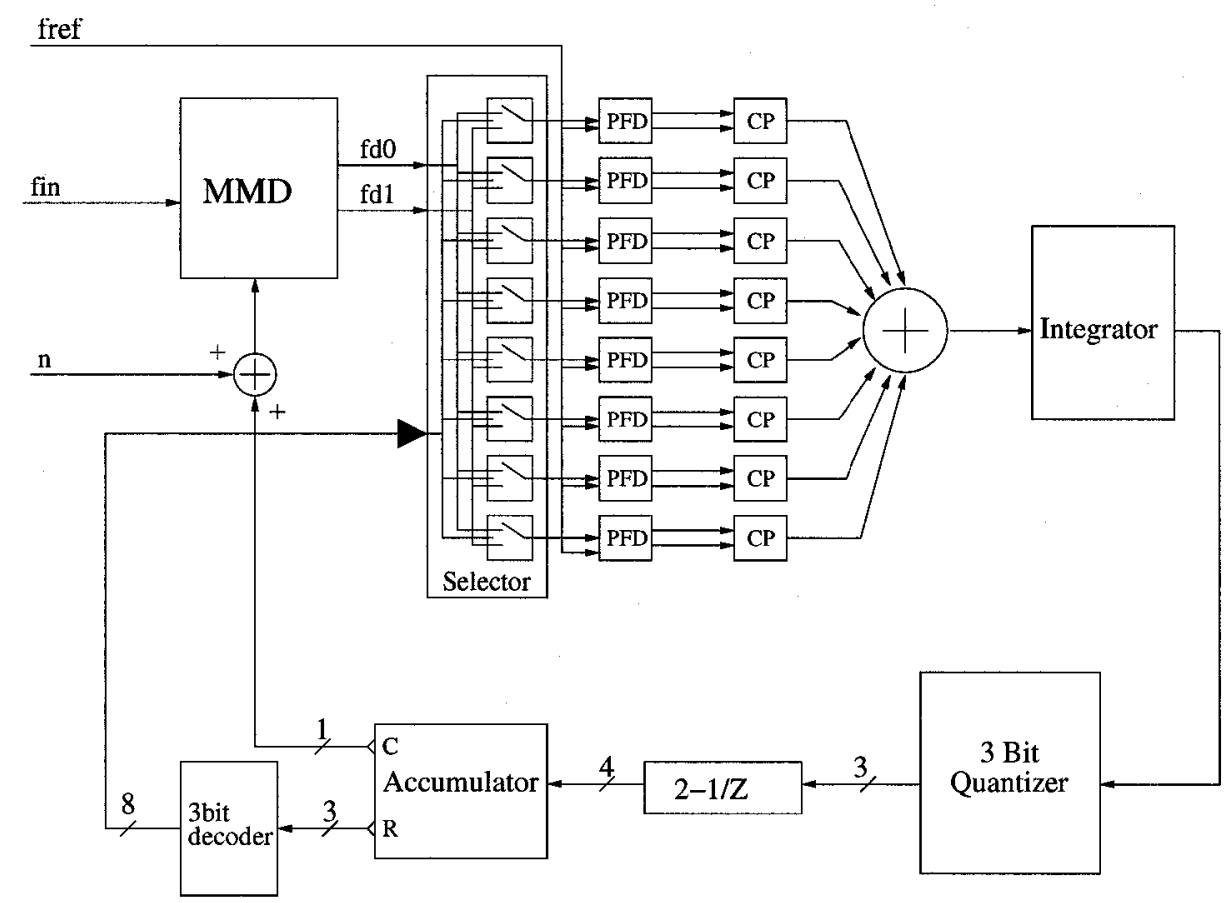

Figure 3.14: Second-Order $\Delta \Sigma$ FD with Fractional-N Divider and Multi-Bit Quantizer

The expression for $Y_{\text {out }}$ is the same as for the single-bit case, but the input frequency 


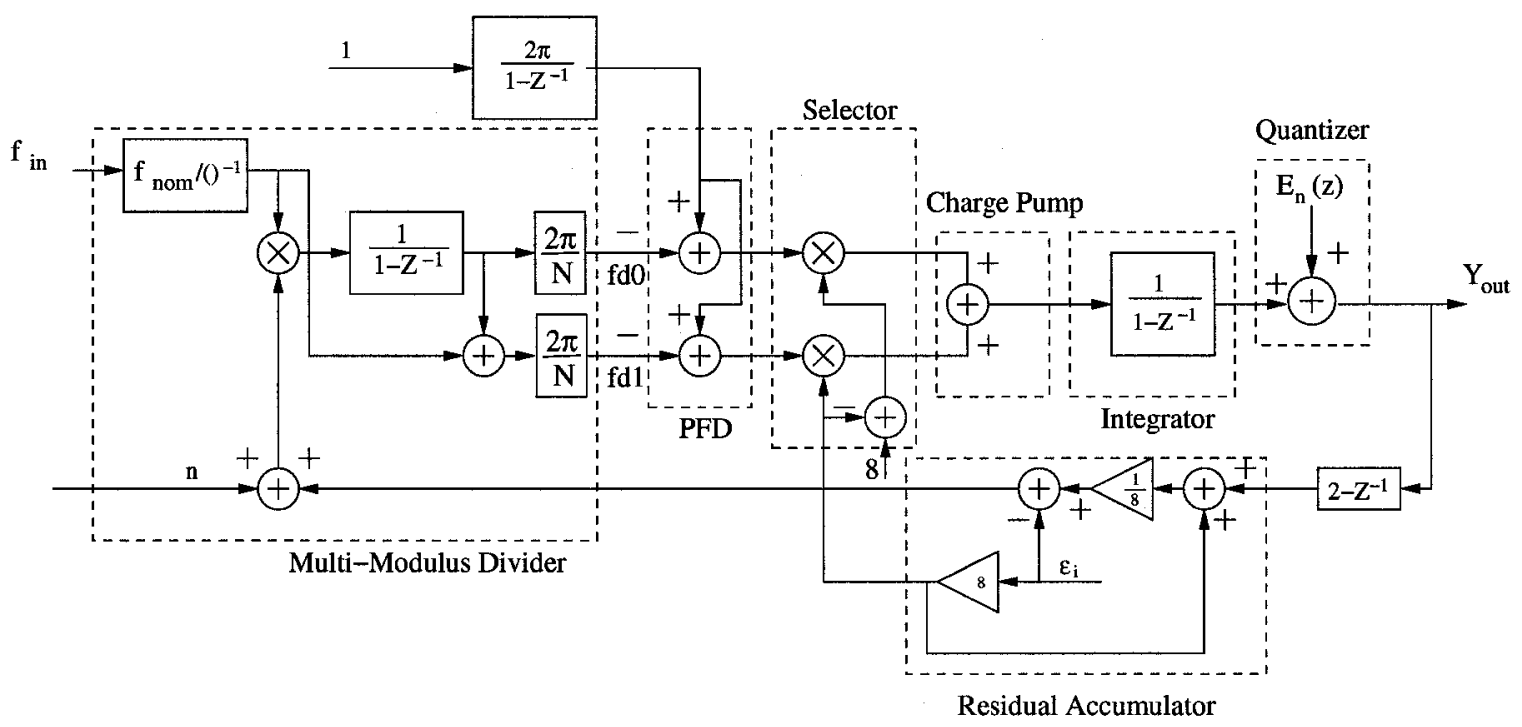

Figure 3.15: Linear Model for Figure 3.14

range is now $\pm \frac{f_{\text {ref }}}{2}$. The size of the input step corresponds to $\frac{1}{8}$ of a input frequency period at the PFD input. 


\subsection{Comparison}

The comparison of the different $\Delta \Sigma \mathrm{FD}$ architectures described in the previous sections is presented in Table.3.1.

\begin{tabular}{|c|c|c|c|}
\hline System Type & Input Range & Quantization Step & $\begin{array}{c}\text { SNR @ } \Omega=50 \mathrm{kHz} \\
\Delta f=-10 \mathrm{~dB} \text { of } \frac{f_{\text {ref }}}{16} \\
10 \mathrm{kHz}-200 \mathrm{kHz} \text { Bandwidth }\end{array}$ \\
\hline 1st Order & $\pm \frac{f_{\text {ref }}}{2}$ & $f_{\text {ref }}$ & $13.71 \mathrm{~dB}$ \\
\hline $\begin{array}{c}\text { 2nd Order } \\
\text { Integer-N }\end{array}$ & $\pm \frac{f_{\text {ref }}}{2}$ & $f_{\text {ref }}$ & $29.15 \mathrm{~dB}$ \\
\hline $\begin{array}{c}\text { 2nd Order } \\
\text { Fractional-N }\end{array}$ & $\pm \frac{f_{\text {ref }}}{16}$ & $\frac{f_{\text {ref }}}{8}$ & $46.68 \mathrm{~dB}$ \\
\hline $\begin{array}{c}\text { 2nd Order } \\
\text { Fractional-N } \\
\text { Multi-Bit }\end{array}$ & $\pm \frac{f_{\text {ref }}}{2}$ & $\frac{f_{\text {ref }}}{8}$ & $46.68 \mathrm{~dB}$ \\
\hline
\end{tabular}

Table 3.1: $\Delta \Sigma$ FD Architecture Performance Comparison

Fig.3.16 shows SNR versus input frequency deviation for cases 2,3 and 4 in Table.3.1.

From table.3.1 and Fig.3.16, it can be seen that the second-order $\Delta \Sigma$ FD with fractional$\mathrm{N}$ divider has higher SNR (about $18 \mathrm{~dB}$ ) than the one with integer-N divider for the same input. By using a 3-bit quantizer, the second-order $\Delta \Sigma$ FD with fractional-N divider has the same full scale input range as the one with integer-N divider. 


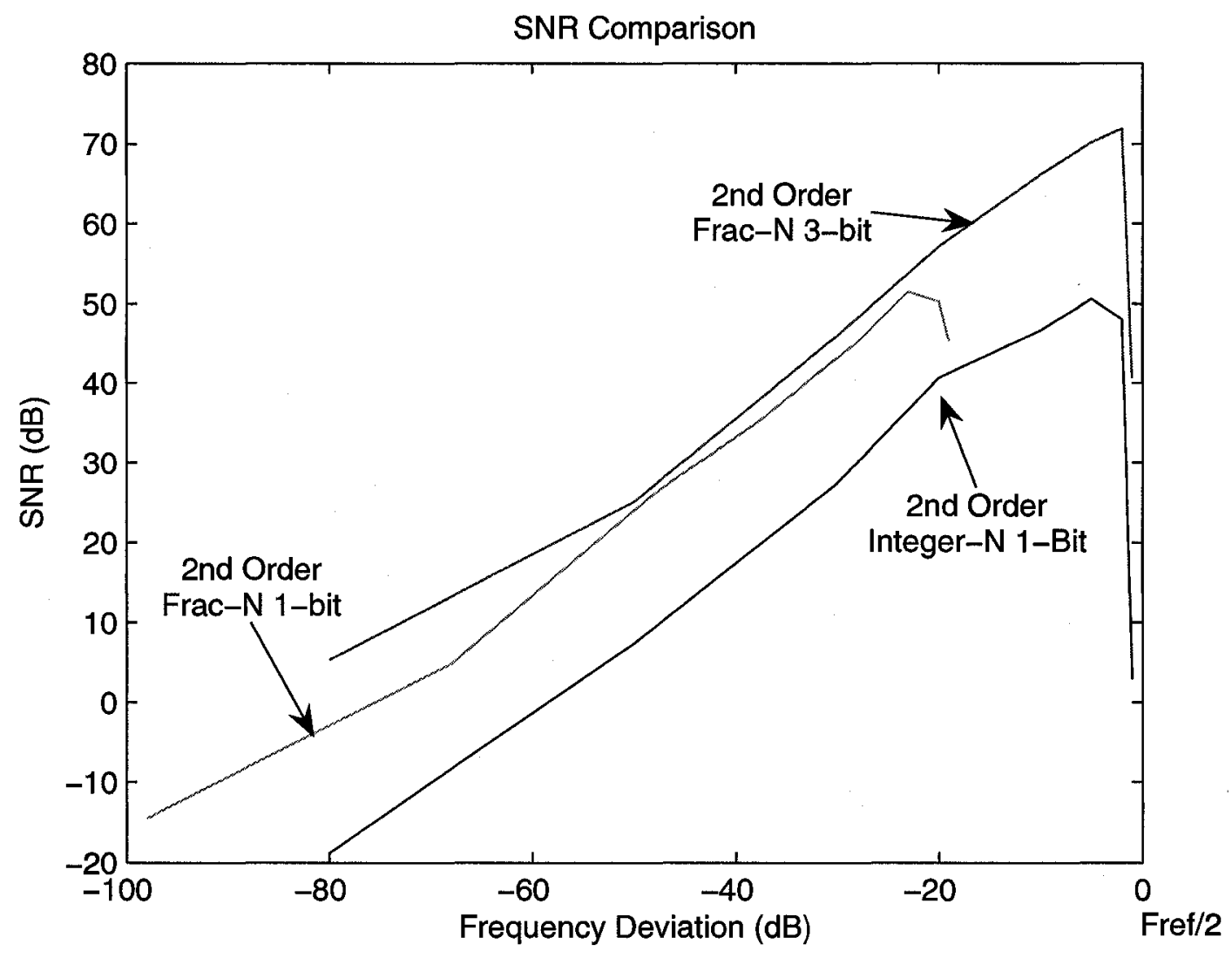

Figure 3.16: SNR vs Input Frequency Deviation 


\subsection{Effects Of System Parameters on Performance}

The system parameters: $f_{\text {ref }}$ (reference clock), $f_{\text {nom }}$ (carrier frequency), $\Delta f$ (maximum frequency deviation), $\Omega$ (modulating frequency) and $n$ (base division ratio) are related. Once the application-dependent values of $f_{n o m}, \Delta f$ and $\Omega$ have been specified, $f_{\text {ref }}$ and $n$ can be determined:

$f_{r e f}$ and $n$ must be chosen so as to satisfy the input range requirement:

$$
n f_{r e f}<f_{\text {nom }} \pm \Delta f<(n+1) f_{\text {ref }}
$$

and $f_{\text {ref }}$ must not violate the Nyquist criterion:

$$
\Omega<\frac{f_{r e f}}{2}
$$

In this thesis, typical values of $f_{n o m}=1 \mathrm{GHz}, \Delta f=1 \mathrm{MHz}$ and $\Omega=50 \mathrm{kHz}$ are chosen as an example. For any given values of $f_{n o m}, \Delta f$ and $\Omega$, there are many $f_{\text {ref }}, n$ combinations that can satisfy Eq.3.24 and Eq.3.25. Four sets of the combinations are chosen to evaluate their influence on system performance. The results are shown in Table.3.2. 


\begin{tabular}{|c|c|c|c|}
\hline$\#$ & $f_{\text {ref }}$ & $n$ & $\begin{array}{c}\text { SNR (simulation) } \\
10 \mathrm{kHz} 200 \mathrm{kHz} \text { Bandwidth } \\
\mathrm{dB}\end{array}$ \\
\hline 1 & $5 \mathrm{M}$ & 200 & 56.40 \\
\hline 2 & $10 \mathrm{M}$ & 100 & 61.57 \\
\hline 3 & $20 \mathrm{M}$ & 50 & 69.87 \\
\hline 4 & $40 \mathrm{M}$ & 25 & 75.05 \\
\hline
\end{tabular}

Table 3.2: Simulation Result for Different Combinations of $f_{r e f}$ and $n$

With each doubling of $f_{\text {ref }}$, the quantization noise PSD $E_{n}^{2}=\frac{\Delta^{2}}{12 f_{s}}$ doubles, but the effects of this doubling are outweighed by the simultaneous doubling of the sampling frequency (see Eq.2.15). The total noise in the band of interest decreases. Therefore, the net effect of increasing $f_{r e f}$ is to improve the SNR. 


\subsection{System Noise Analysis}

System noise is an important performance determinant and a careful analysis is essential to an evaluation of the new architecture's feasibility. Such an analysis will allow noise sources and their relative importance to be identified so that design efforts can be appropriately focused.

The noise generated in each block of this architecture is modified by a characteristic transfer function, such as a high-pass or a low-pass one. In preparation to analyzing the effects of noise in different blocks, the new architecture's linear model (Fig.3.15) is redrawn as Fig.3.17.

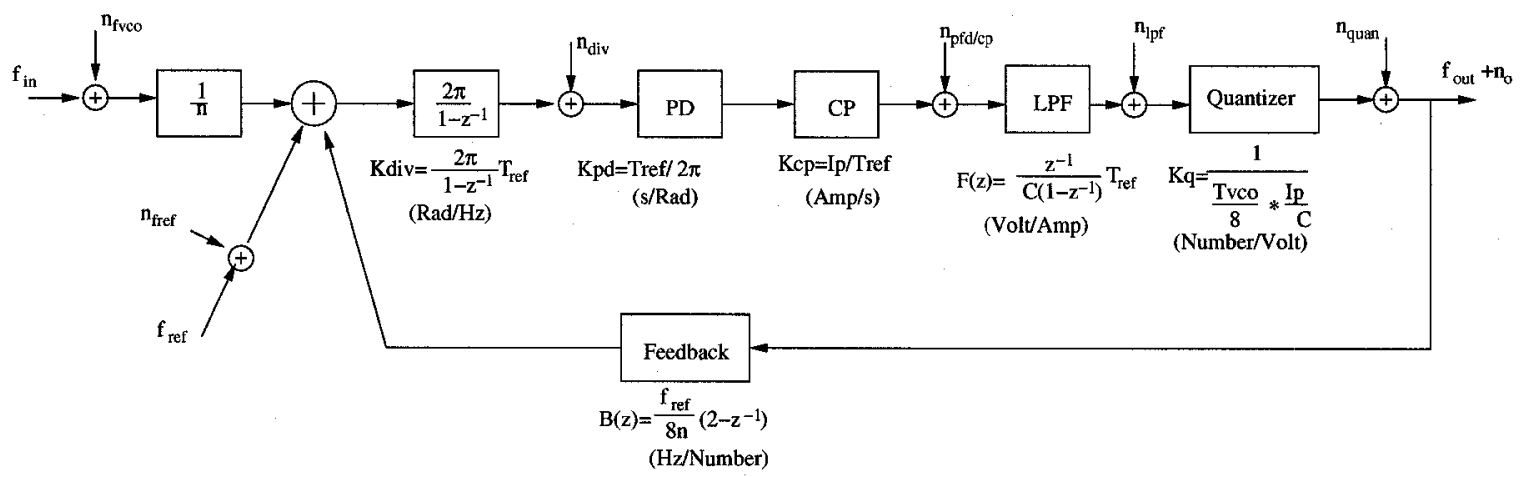

Figure 3.17: Linear Model of 2nd-Order Fractional-N $\Delta \Sigma$ FD

In the interest of clarity, the original figure has been condensed, with the functions of the circuit blocks being depicted mathematically.

In each block, information is carried in different formats. The input to the $\Delta \Sigma \mathrm{FD}$ (in particular, the divider) takes the form of frequency in units of hertz. The divider functions as a perfect integrator whose output is in units of radians. The PFD generates pulses with their widths, in units of seconds, proportional to the phase difference at the input of the PFD. The charge pump produces a current pulse, with units of amperes, that has a duration 
that is proportional to the width of the pulse from the PFD. At the output of the integrator, the information is carried in the form of voltage, in units of volts, which is proportional to the current produced by the charge pumps. The quantizer digitizes the voltage and represents the information in discrete numbers. The feedback loop converts the number back to frequency. The system compares the feedback output with the input frequency and tries to minimize the error.

The system output in Fig.3.17 is given by:

$$
f_{\text {out }}=\left(\frac{f_{\text {in }}}{n}-f_{\text {ref }}\right) \frac{K_{d i v} K_{p f d} K_{c p} F(z) K_{q u a n}}{1+K_{d i v} K_{p f d} K_{c p} F(z) K_{q u a n} B(z)}=8 n\left(\frac{f_{\text {in }}}{n f_{r e f}}-1\right)
$$

Where,

- $K_{d i v}=\frac{2 \pi}{1-z^{-1}} T_{r e f}$ is the transfer function of divider, in units of radians/hertz.

- $K_{P F D}=\frac{T_{r e f}}{2 \pi}$ is the gain of the PFD, in units of seconds/radian.

- $K_{C P}=\frac{I_{p}}{T_{r e f}}$ is the gain of the CP, in units of amperes/second.

- $F(z)=\frac{z^{-1}}{C\left(1-z^{-1}\right)} T_{r e f}$ is the transfer function of integrator, in units of volts/ampere.

- $K_{q}=\frac{1}{\frac{T_{i n} \frac{I_{p}}{8}}{C}}$ is the gain of the quantizer, in units of numbers/volt.

- $B(z)=\frac{f_{\text {in }}}{8 n}\left(2-z^{-1}\right)$ is the transfer function of the feedback block, in units of hertz/number.

- $I_{p}$ is the source current of the charge pump, in unit of ampere.

- $C$ is the integrator capacitor value, in units of farads.

The output $f_{\text {out }}$ is in form of discrete numbers from 0 to 7 representing the actual frequency deviation from 0 to $f_{r e f}$.

The transfer functions seen by noise signals at various points in the system will now be developed. 


\subsubsection{Quantization Noise Transfer Function}

Letting $n_{o}$ be the output noise power, and $n_{\text {quan }}$ the quantizer noise power, we have

$$
\frac{n_{o}}{n_{\text {quan }}}=\frac{1}{1+A_{o l}}
$$

Where $A_{o l}$ is the open loop gain:

$$
A_{o l}=K_{d i v} K_{p f d} K_{c p} F(z) K_{q} B(z)=\frac{\left(2-z^{-1}\right) z^{-1}}{\left(1-z^{-1}\right)^{2}}
$$

Thus,

$$
\frac{n_{o}}{n_{\text {quan }}}=\left(1-z^{-1}\right)^{2}
$$

\subsection{2 $f_{\text {in }}$ Noise Transfer Function}

$$
\frac{n_{o}}{n_{f_{\text {in }}}}=\frac{1}{n} \frac{K_{d i v} K_{p f d} K_{c p} F(z) K_{q}}{1+A_{o l}}=\frac{8}{f_{r e f}} z^{-1}
$$

\subsection{3 $f_{\text {ref }}$ Noise Transfer Function}

$$
\frac{n_{o}}{n_{f_{r e f}}}=\frac{K_{d i v} K_{p f d} K_{c p} F(z) K_{q}}{1+A_{o l}}=\frac{8 n}{f_{r e f}} z^{-1}
$$

\subsubsection{Divider Noise Transfer Function}

$$
\frac{n_{o}}{n_{d i v}}=\frac{K_{p f d} K_{c p} F(z) K_{q}}{1+A_{o l}}=\frac{8 n}{2 \pi}\left(1-z^{-1}\right) z^{-1}
$$




\subsubsection{PFD and CP Noise Transfer Function}

The noise originating in the PFD and CP blocks is combined, as measurement or simulation of the noise at the phase detector output is difficult.

$$
\frac{n_{o}}{n_{P F D \_C P}}=\frac{F(z) K_{q}}{1+A_{o l}}=\frac{8 n}{I_{p}}\left(1-z^{-1}\right) z^{-1}
$$

\subsubsection{Integrator Noise Transfer Function}

$$
\frac{n_{o}}{n_{\text {int }}}=\frac{K_{q}}{1+A_{o l}}=\frac{8 n}{\frac{I_{p}}{c} T_{r e f}}\left(1-z^{-1}\right)^{2}
$$

\subsubsection{Quantizer Noise Transfer Function}

The quantizer circuit noise is treated in the same manner as quantization noise, as shown in Eq.3.27.

All of the NTFs (Noise Transfer Functions) are plotted in Fig.3.18. The system parameters used to obtain these are:

$$
n=100, f_{r e f}=10 \mathrm{MHz}, I_{p}=200 u A, C=1 p F
$$

Fig.3.18 shows that noise from $f_{\text {in }}$ and $f_{\text {ref }}$ has no shaping. Noise from the divider, PFD and CP are subject to first-order shaping, and those from the integrator and quantizer are subject to second-order shaping.

Later, in Chapter 4, these transfer functions will be used to predict the effect of simulated circuit noise on the system. 


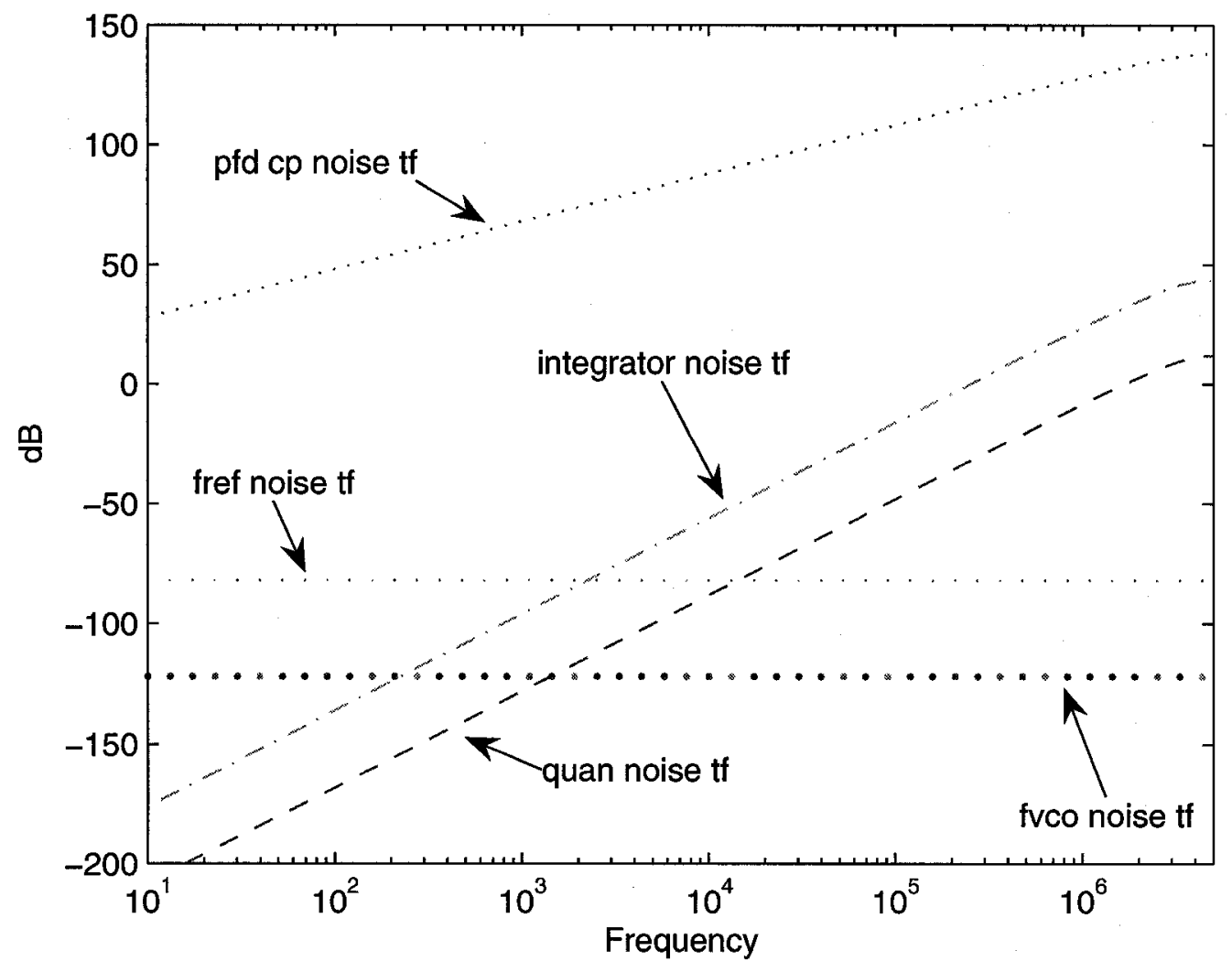

Figure 3.18: Noise Transfer Function at Various Circuit Nodes

\subsection{Circuit Performance Improvement Techniques}

In this section, some system level approaches to minimize the adverse affects of circuit non-idealities are described.

CMOS VLSI processes are typically optimized for high-density digital design and not high precision analog design. Mismatches between the charge pumps and PFDs is inevitable, and can lead to increased in-band noise levels and reduced SNR.

A technique called Data Weighted Averaging (DWA) has been exploited in this design 
to lessen these mismatch effects. This technique [BF95] [NH96] performs a first-order shaping of the mismatch caused distortion, and moves noise to higher frequencies where it can be removed by filtering.

Figure 3.19 shows the fundamental principle behind this technique, using the simple case of a 2-element CP array. To represent the effect of CP output error, each of the two CPs has an output $10 \%$ above or below the desired current. The output current errors will average to zero when both CPs contribute to the output, but if only one contributes, a net $10 \%$ error will appear in the output as illustrated in Fig.3.19. This suggests that errors resulting from the non-ideal implementation of analog circuits can be reduced if the outputs of several CPs are averaged to produce the final output.

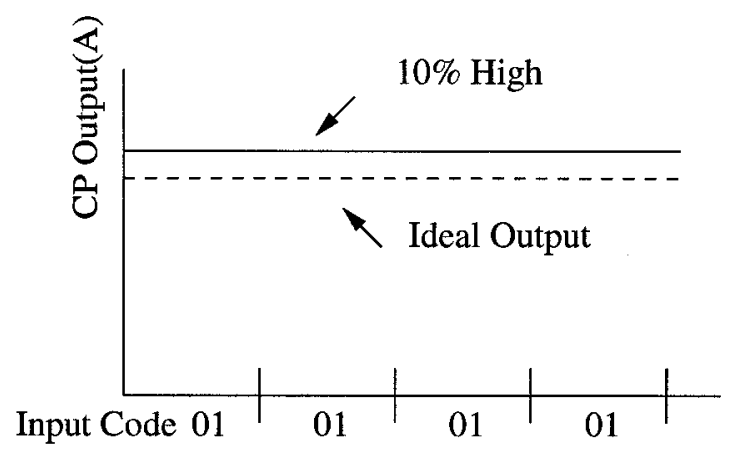

(a)

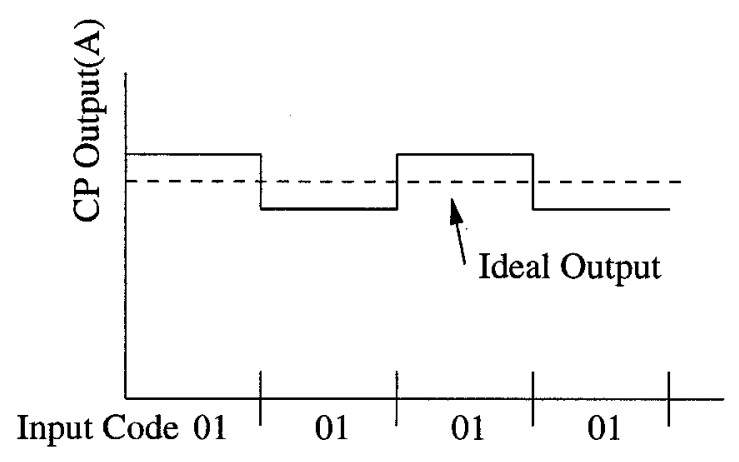

(b)

Figure 3.19: CP Output with (a) no DWA, and (b) DWA 
The element selection process used in DWA must ensure that the work is apportioned equally among the CPs in the array. This is done by a cyclic element selection process whose concept of operation is illustrated in Fig.3.20 [BF95] [NH96].

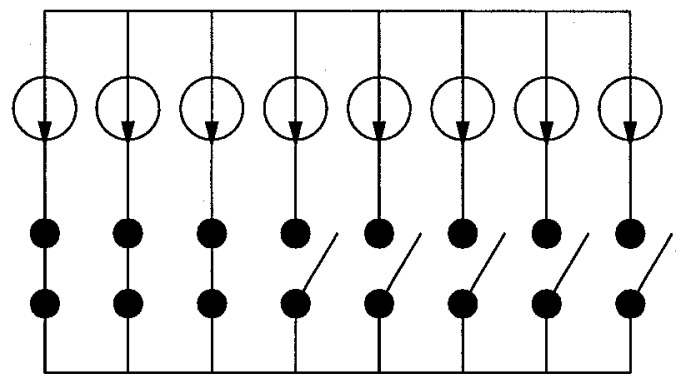

(a)

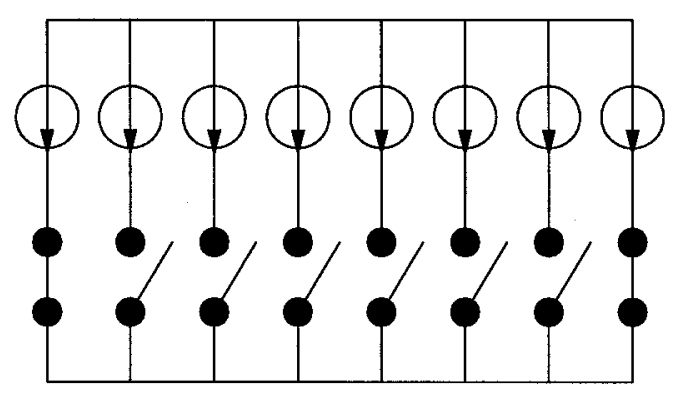

(c)

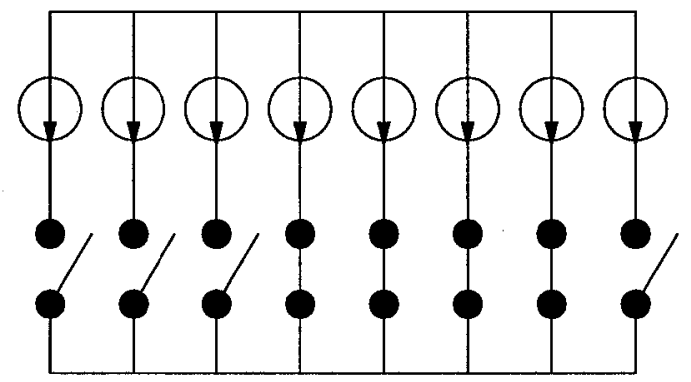

(b)

Figure 3.20: DWA for Eight Elements - Input Sequence: 011, 100 and 010

In the figure above, an array of eight CPs is used. For each new input sequence, the elements that are adjacent to those used in the previous input sequence will be selected. To express this algorithm mathematically, one can begin by imagining an infinite element array and a pointer $p t r(k)$ containing the address of the first cell of the $(k+1)_{t h}$ selection. At each clock cycle, this pointer is incremented by the input code $x(k)$ :

$$
\operatorname{ptr}(k)=\operatorname{ptr}(k-1)+x(k)
$$


The mismatch error at cycle $k$ is

$$
y_{m i s}(k)=\sum_{i=p t r(k-1)}^{p t r(k)-1} w_{i}-x(k) w_{\text {mean }}
$$

Where the $w_{i}$ are the 'weights' of the elements and $w_{\text {mean }}$ their average

$$
w_{\text {mean }}=\frac{1}{N} \sum_{i=0}^{N-1} w_{i}
$$

In the absence of mismatches, $w_{0}=w_{1}=\ldots=w_{N-1}$.

$\mathrm{Eq}(3.35)$ can be re-written in the form:

$$
y_{m i s}(k)=I M(p t r(k))-I M(p t r(k-1))
$$

Where $I M(p t r)$ is the Integral Mismatch Function [BF95] [NH96].

$$
I M(p t r)=\sum_{i=0}^{p t r-1}\left(w_{i}-w_{\text {mean }}\right)
$$

$y_{m i s}(k)$ is obtained by first-order differentiation of the function $I M(p t r)$ and so benefits from first-order noise shaping. It has been proven that for random input signals, $I M(p t r)$ has a white spectrum [NH96].

The circuit implementation for the DWA block is described in Chapter 4.

Simulations of the second-order $\Delta \Sigma$ FD with fractional-N divider and multi-bit quantizer were run with three cases: no $\mathrm{CP}$ mismatch, $\mathrm{CP}$ mismatch $10 \%$ with DWA and CP mismatch without DWA. The results are captured in Fig.3.21. The in-band first-order shaping on noise from the CP mismatch reduces the total in-band noise, and therefore increases SNR. 


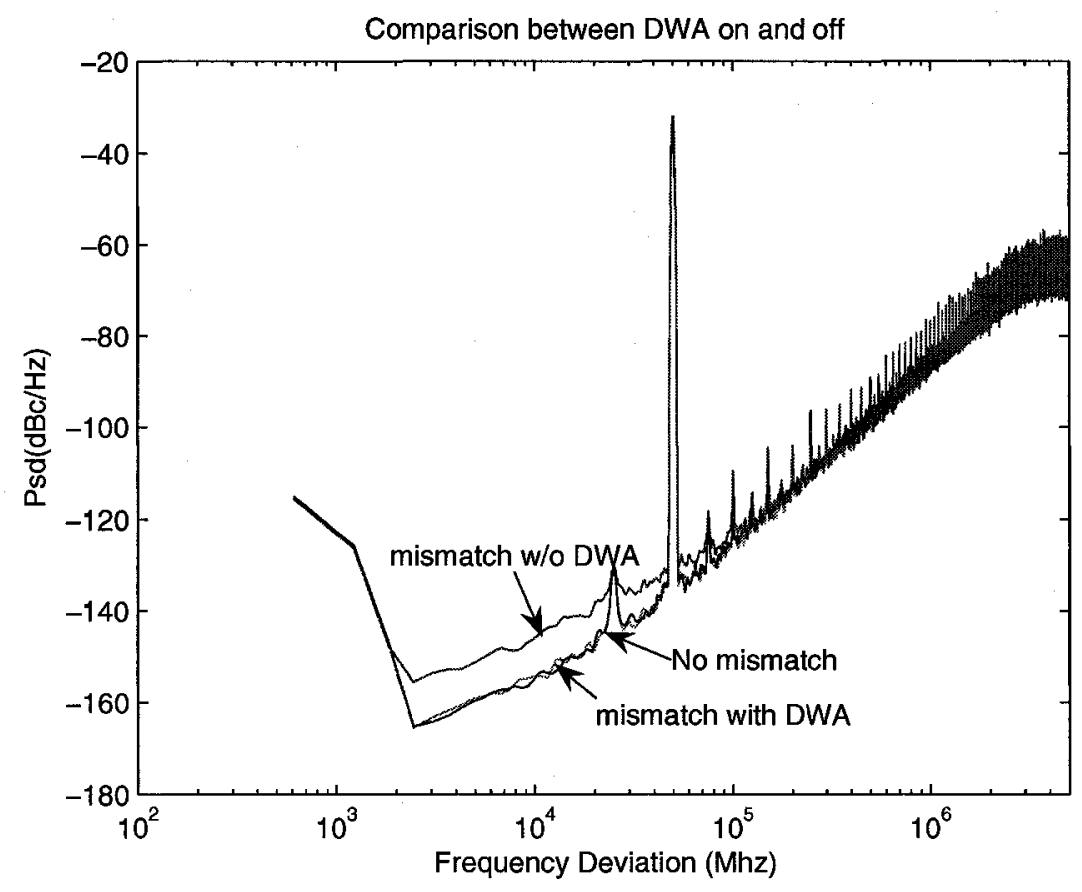

Figure 3.21: Comparison between no CP Mismatch, CP Mismatch with and without DWA

\subsection{Summary}

In this chapter, a system level analysis of different $\Delta \Sigma$ FD architectures was presented. In the next chapter, the circuit level implementation of the new architecture is presented. 


\section{Chapter 4}

\section{Circuit Design and Simulation Results}

This chapter describes the $\Delta \Sigma$ FD design at the circuit level using a TSMC $0.18 \mu \mathrm{m}$ CMOS process. The design goal is validation of the system architecture discussed in Chapter 3 , and not to create a circuit optimized for lowest power consumption, lowest noise, or smallest area.

The partitioning of the system into circuit blocks is shown in Fig.4.1.

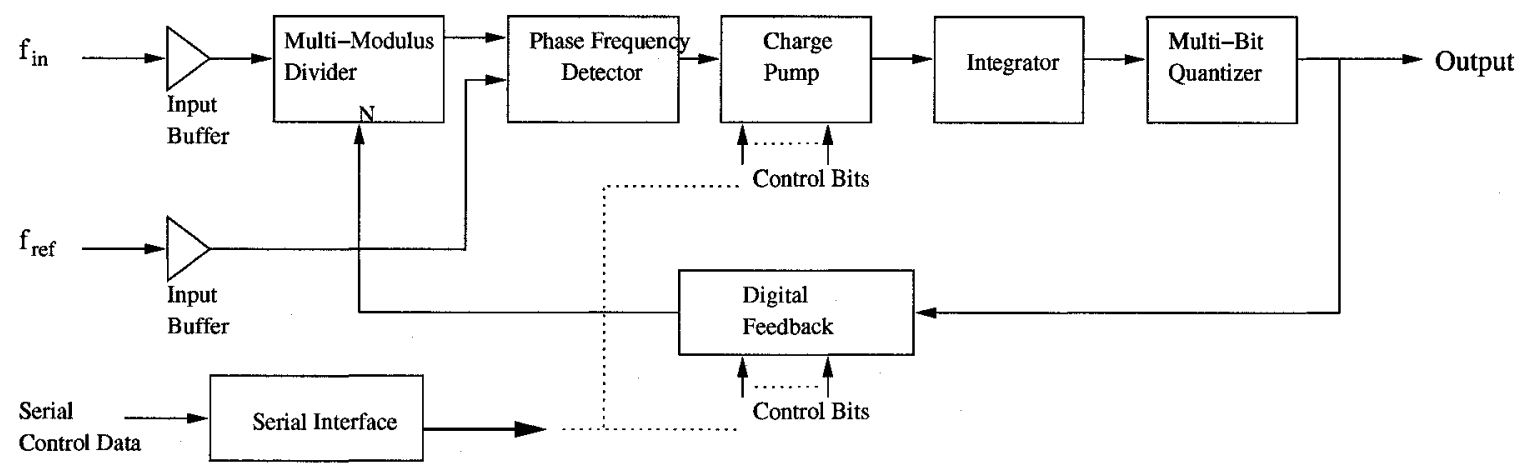

Figure 4.1: System Block Diagram

The system has two inputs: $f_{\text {in }}$, a frequency-modulated carrier and $f_{r e f}$, a $10 \mathrm{MHz}$ ref- 
erence clock. $f_{\text {in }}$ is chosen to fall in the range $n f_{r e f}<f_{\text {in }}<(n+1) f_{r e f}$, where $n$ is the base divisor of the MMD.

The circuit operates as follows: The PFD compares the phase of $f_{d i v}$, the divided down version of $f_{i n}$, to that of $f_{r e f}$ and generates two pulses, $P U$ and $P D$, which are used as inputs to the CP. The difference between the width of these two pluses is proportional to the phase difference between $f_{d i v}$ and $f_{r e f}$. The $\mathrm{CP}$ acts as a current source when $P U$ is high and a current sink when $P D$ is high. The net current is converted to a voltage by the integrator, whose output is sampled by the 3-Bit Quantizer. The quantizer's 3-Bit output, which is also the $\Delta \Sigma$ FD 's output, is used by the Digital Feedback block to generate the next MMD divisor. The feedback loop is closed by sending the new $N$ to the MMD.

The output of the system is the 3-Bit data stream from the Quantizer and represents the deviation of $f_{\text {in }}$ from its nominal constant carrier frequency.

This system is a mixed-signal system with digital and analog components working together to perform the overall system function. The relationship between the analog and digital design flows is illustrated in Fig.4.2. The design tools used at each of the indicated stages are also named.

Implementation details of each circuit block are described in the following sections. 


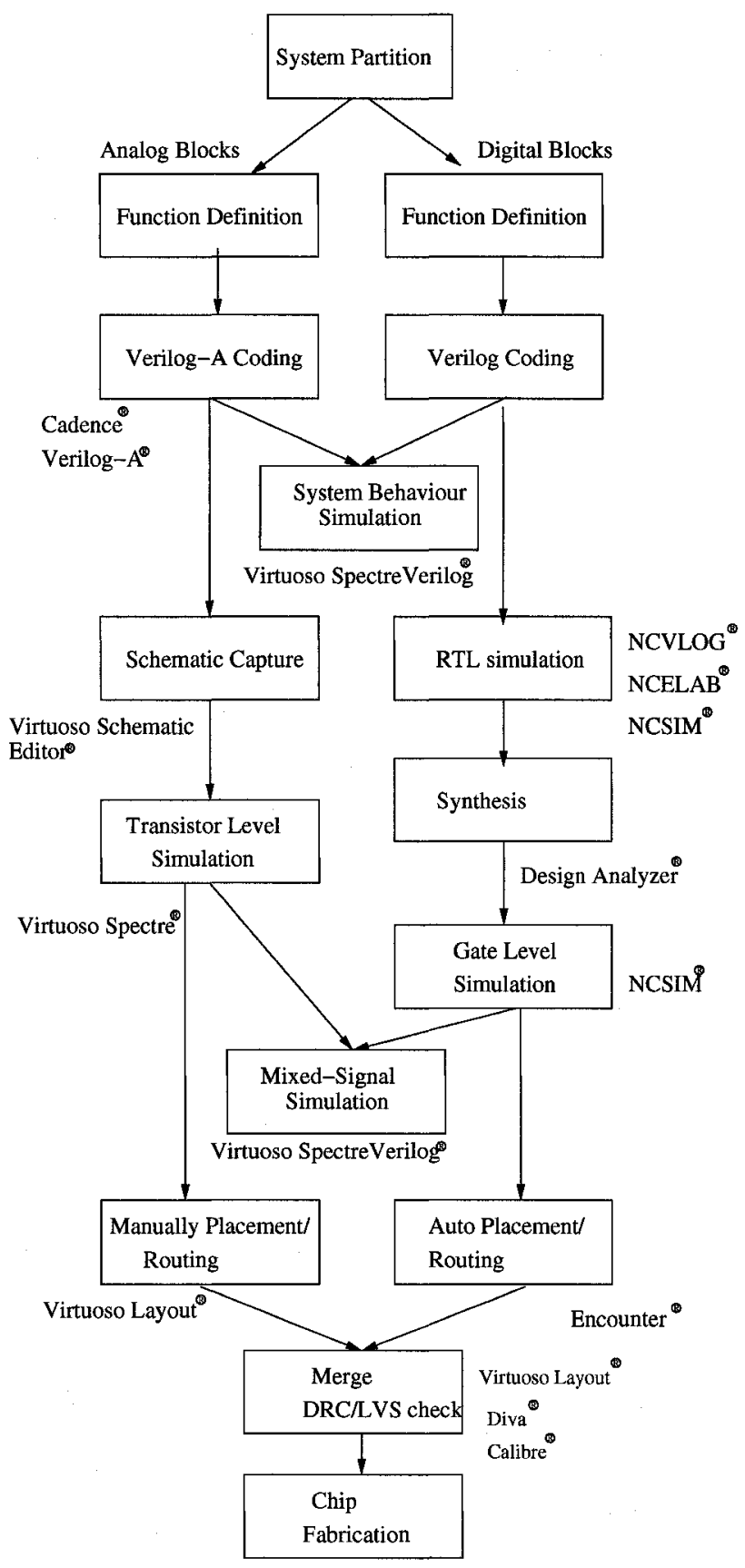

Figure 4.2: $\Delta \Sigma$ FD Design Flow and Tools Used 


\section{1 $f_{\text {in }}$ and $f_{r e f}$ Input Buffers}

These input buffers convert applied analog sinusoidal waveforms to digital (CMOS) levels. The simplified schematic used for both buffers is shown in Fig.4.3.

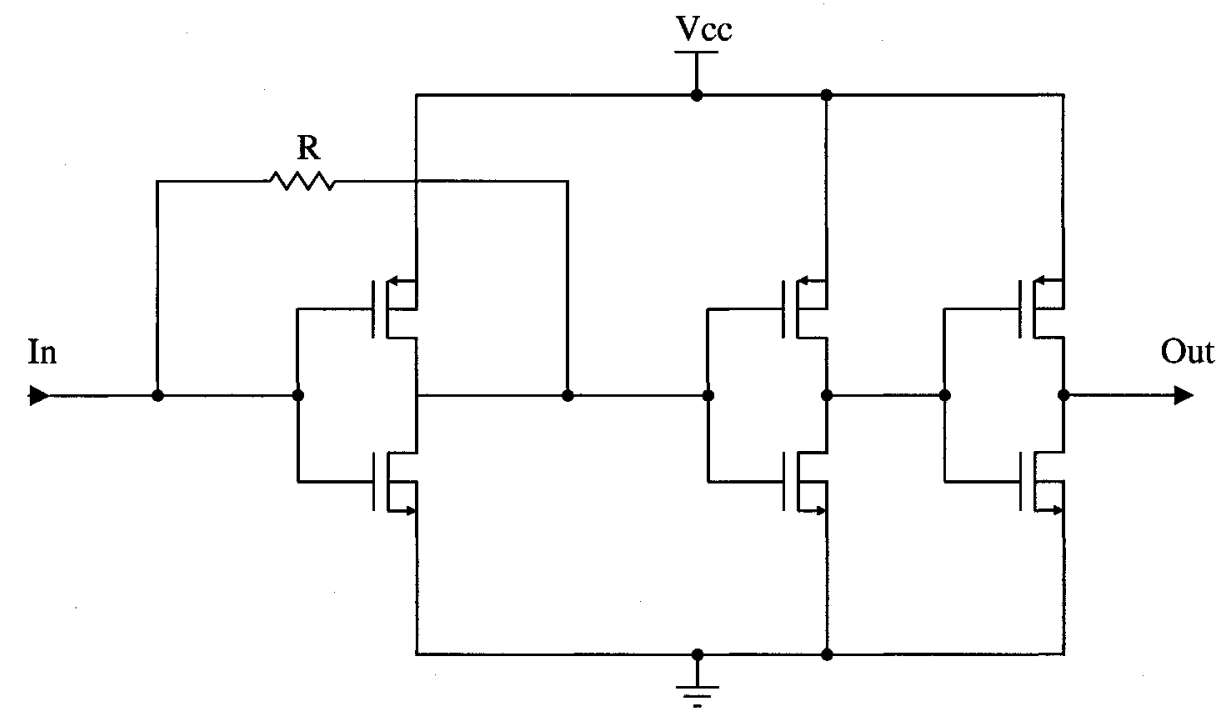

Figure 4.3: Input Buffer

Feedback is used to linearize the first stage and so reduce the input noise gain. Furthermore, a larger $\frac{W}{L}$ was used in the first stage to reduce the circuit-generated noise. In principle, greater channel area means less $\frac{1}{f}$ noise and thermal noise, but requires more current for speed,

The design of the $f_{\text {in }}$ input buffer differs from that of the $f_{\text {ref }}$ buffer only in transistor sizing. Device sizes need to be scaled down because of the higher frequencies involved. 


\subsubsection{Simulation Results}

Operation of the $f_{r e f}$ and the $f_{\text {in }}$ input buffers was simulated using Spectre. The simulation results are shown in Fig.4.4 and Fig.4.5.

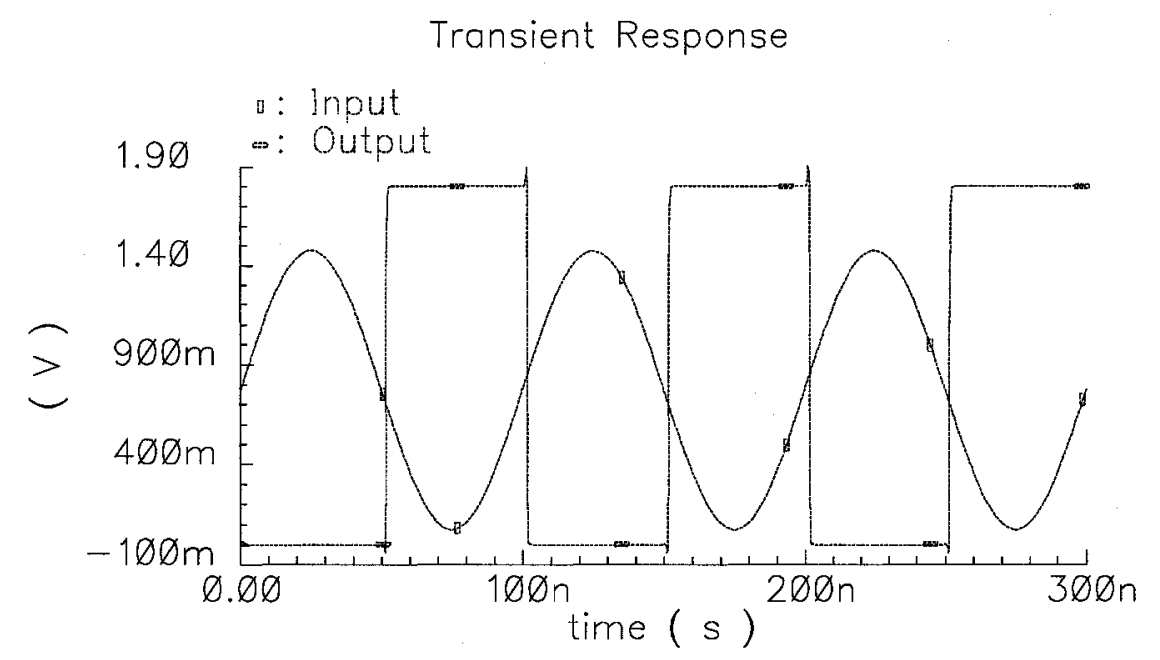

Figure 4.4: $f_{\text {ref }}$ Input Buffer Operation 


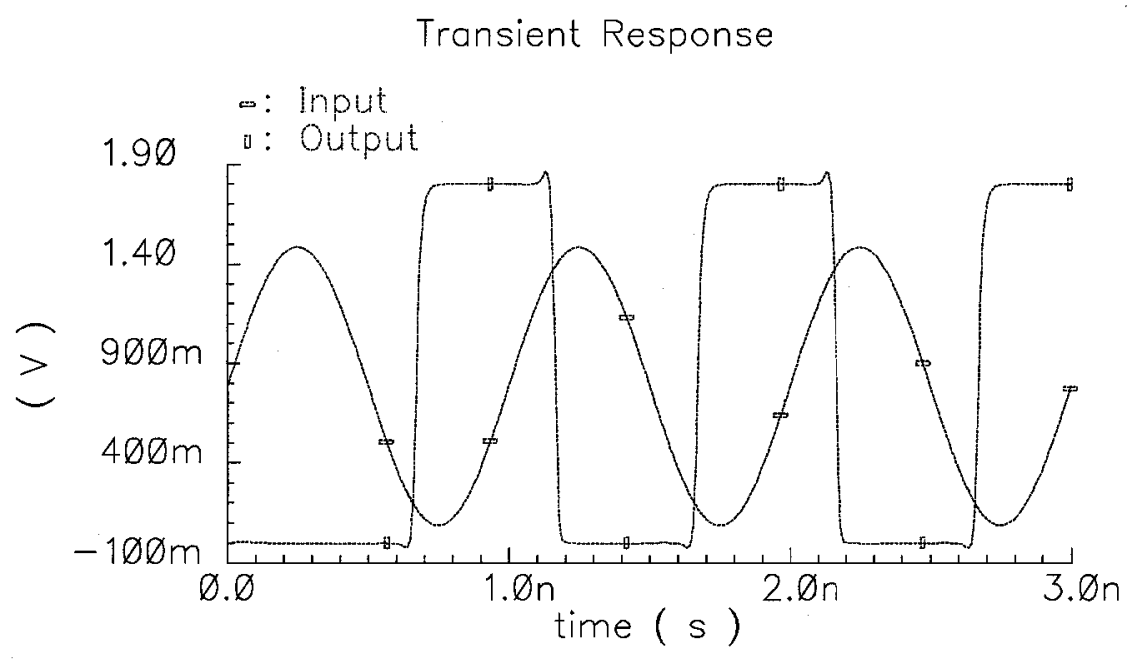

Figure 4.5: $f_{\text {in }}$ Input Buffer

\subsubsection{Phase Noise Performance}

The phase noise seen at the buffers' output has two sources - one source is the phase noise already present in the input signal, about which nothing can be done, and the second source is that generated internally by the buffer. A design goal is that phase noise added by the input buffer must be $3 \mathrm{~dB}$ below the specified input phase noise. A typical low noise 10 $\mathrm{MHz}$ reference clock produced by a signal generator, HP8663A for example, has a phase noise of $-147 \mathrm{dBc} / \mathrm{Hz} @ 10 \mathrm{kHz}$.

Spectre PSS simulation was used to predict the phase noise spectra at the $f_{\text {ref }}(10 \mathrm{MHz})$ and $f_{\text {in }}(1 \mathrm{GHz})$ buffer outputs.The results are shown in Fig.4.6 and Fig.4.7, respectively.

Fig.4.6 shows that noise added by the $f_{\text {ref }}$ input buffer is much less than that present in the input signal.

The relative importance of the phase noise present in the $f_{\text {ref }}$ and $f_{\text {in }}$ input signals will be evaluated later in this chapter. 
Periodic Noise Response

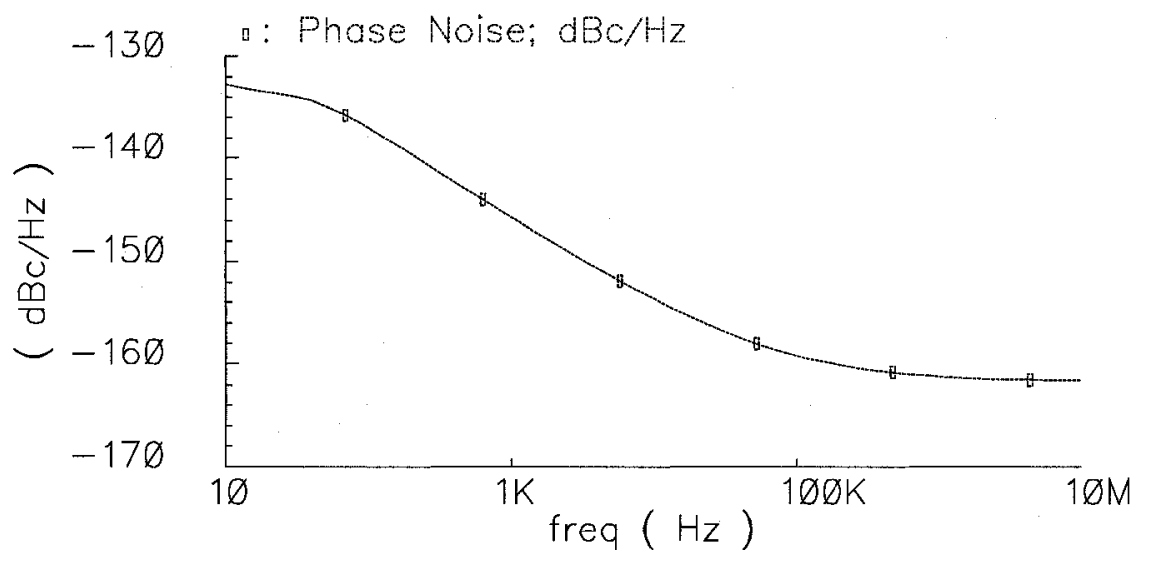

Figure 4.6: Output Phase Noise of $f_{\text {ref }}$ Buffer

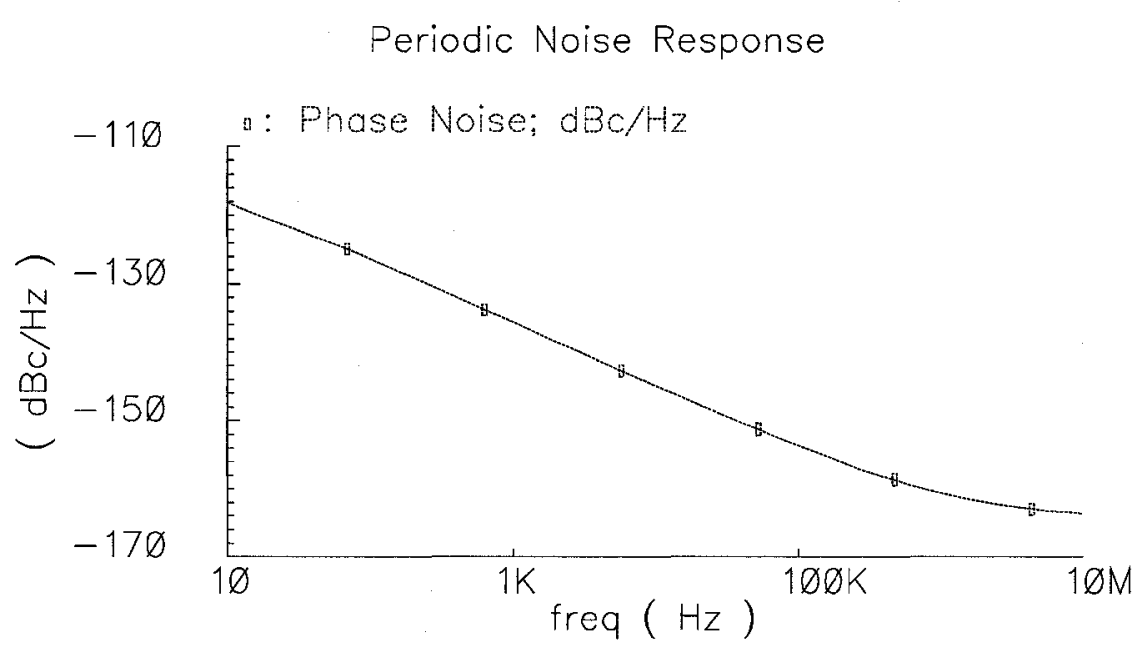

Figure 4.7: Output Phase Noise of $f_{\text {in }}$ Buffer 


\subsection{Programmable Multi-Modulus Divider}

The programmable Multi-Modulus Divider (MMD) divides the input frequency by a divisor determined by the output of the Digital Feedback block. The MMD structure used in this work, one consisting of a Prescaler and two down-counters, is shown in Fig.4.8.

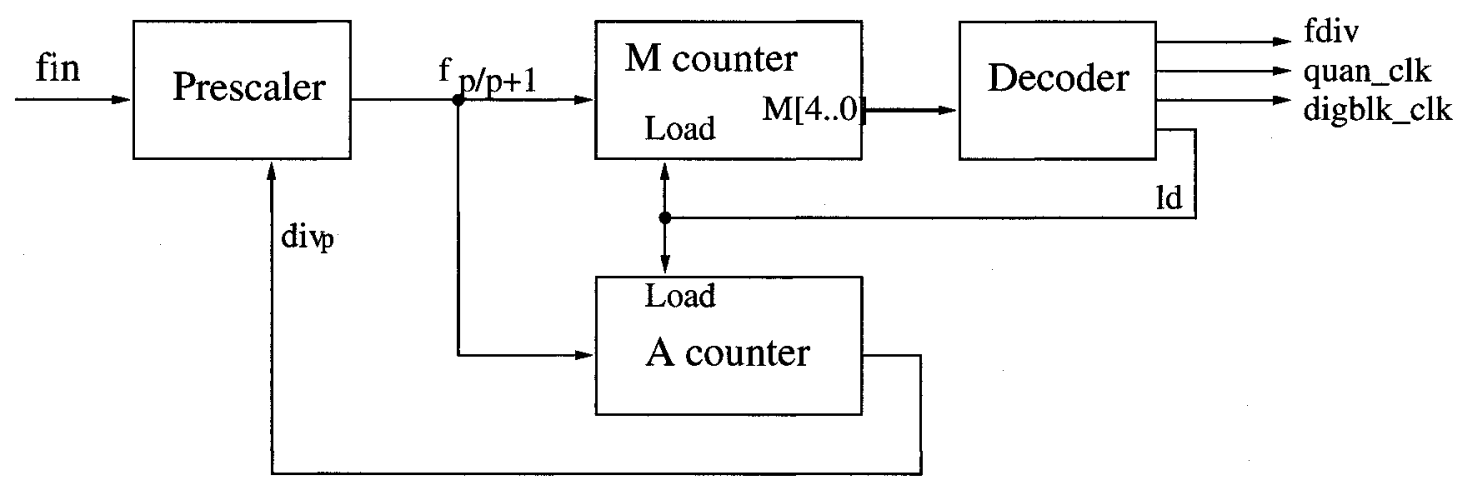

Figure 4.8: Programmable Multi-Modulus Divider

The MMD works by dividing $f_{\text {in }}$ by a number $P+1$ for a period of time and then by $P$ for a period of time. The total time for these operations is one cycle of the output frequency, $f_{d i v}$.

Let the first period of time be equal to A periods of a waveform whose frequency is $\frac{f_{i n}}{P+1}$ and the second be 'M-A' periods of a waveform with frequency $\frac{f_{i n}}{P}$.

The total time required for the two-modulus division is $T_{d i v}=\frac{A}{f_{i n} /(P+1)}+\frac{M-A}{f_{i n} / P}$ seconds, or $T_{d i v}=\frac{A+M P}{f_{i n}}$ seconds. The output frequency of the divider can be given by:

$$
f_{d i v}=\frac{f_{\text {in }}}{N}
$$

Where $N=A+M P, f_{d i v}=\frac{1}{T_{d i v}}$. 
The timing diagram for the operation of the MMD is shown in Fig.4.9,

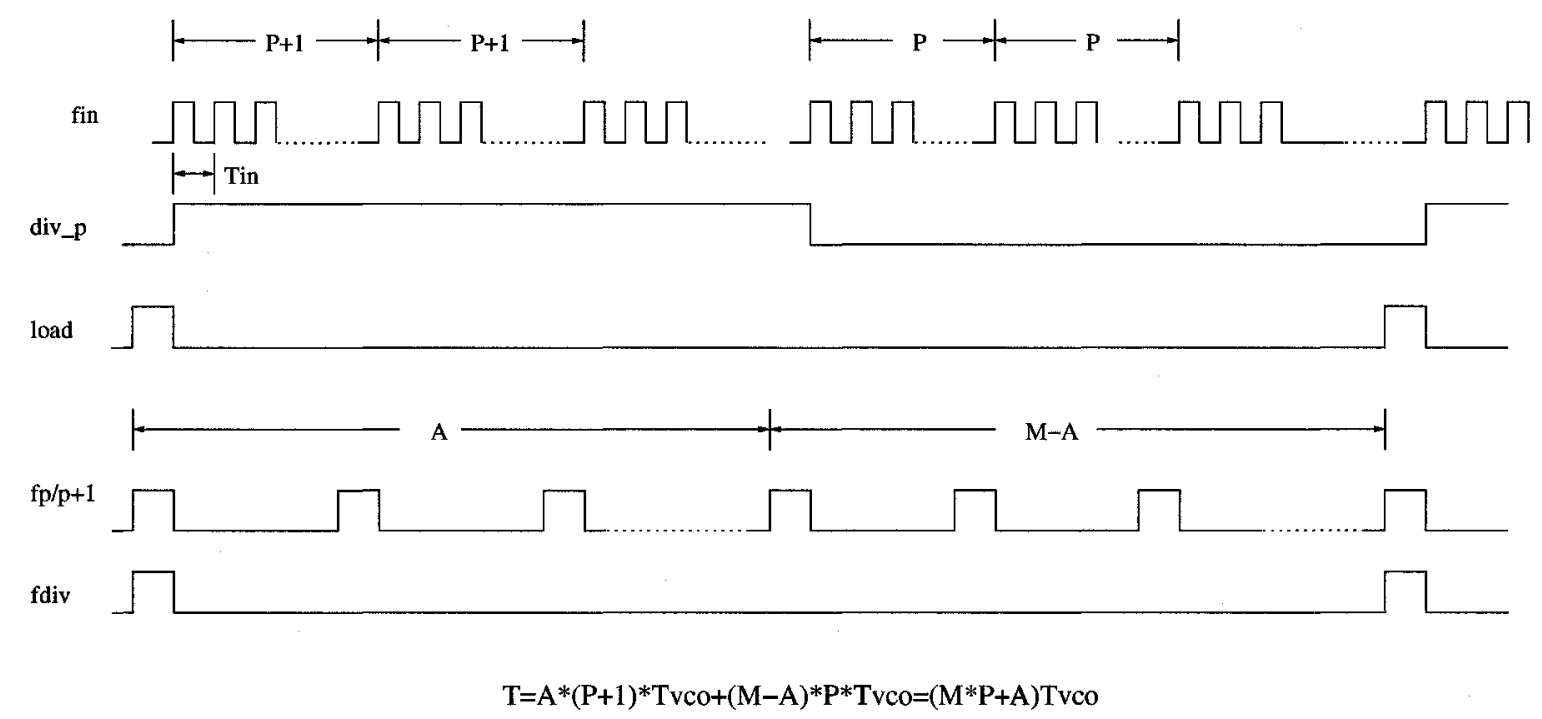

Figure 4.9: Programmable MMD Timing

In this thesis, a 5-bit M-counter and a 2-bit A-counter are used. A value of 4 for $P$ was chosen so that the divisor components can be expressed in the simple binary format $\left(N=M_{4} M_{3} M_{2} M_{1} M_{0} A_{1} A_{0}=4 M+A\right)$.

\subsubsection{Prescaler}

The prescaler divides $f_{\text {in }}$ by either $4(P)$ or $5(P+1)$. The divisor is determined by the state of the $d i v_{p}$ input: $\operatorname{div} p=0[1] \rightarrow$ divide by $4[5]$

The schematic for the Prescaler is shown in Fig.4.10.

The state table for the Prescaler is shown in Table.4.1. 


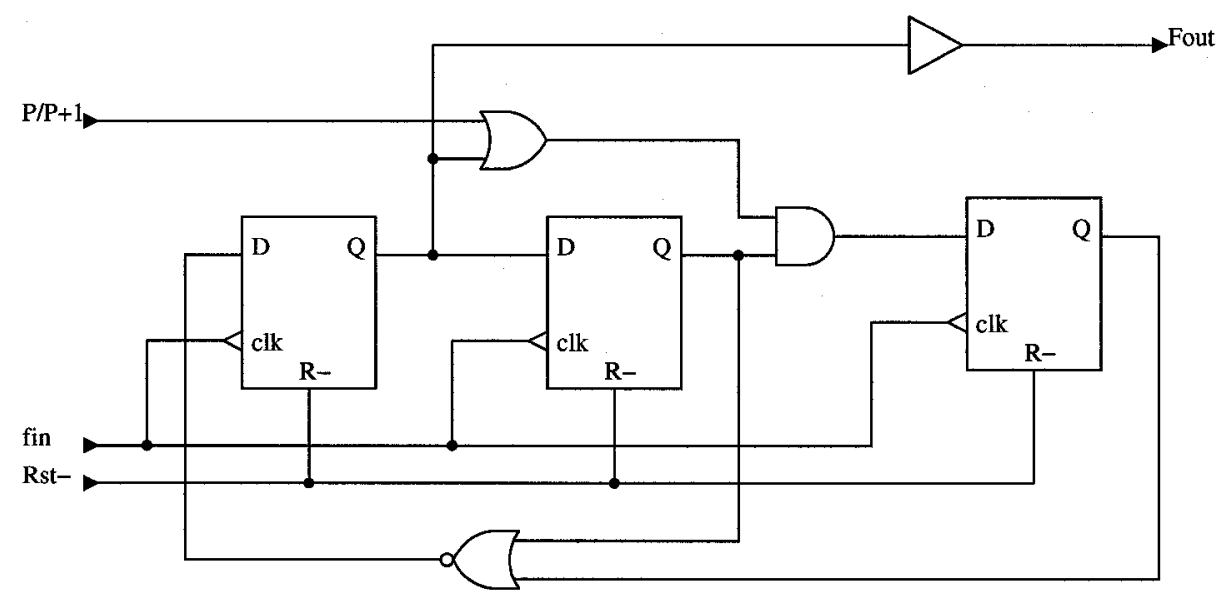

Figure 4.10: Schematic for Prescaler

\begin{tabular}{|l|l|l|l|l|l|l|}
\hline $\begin{array}{l}\text { Present } \\
\text { state }\end{array}$ & $\begin{array}{l}\text { Next state } \\
\text { divide by } 4\end{array}$ & $\begin{array}{l}\text { Present } \\
\text { state }\end{array}$ & $\begin{array}{l}\text { Next State } \\
\text { divide by } 5\end{array}$ & $\begin{array}{l}\text { Invalid } \\
\text { state }\end{array}$ & $\begin{array}{l}\text { Next State } \\
\text { divide by } 4\end{array}$ & $\begin{array}{l}\text { Next State } \\
\text { divide by } 5\end{array}$ \\
\hline 000 & 001 & 000 & 001 & 010 & 000 & 100 \\
\hline 001 & 011 & 001 & 011 & 101 & 010 & 010 \\
\hline 011 & 110 & 011 & 110 & 111 & 110 & 110 \\
\hline 110 & 000 & 110 & 100 & 100 & 000 & \\
\hline & & 100 & 000 & & & \\
\hline
\end{tabular}

Table 4.1: Prescaler State Table

\subsubsection{M-Counter}

After the M-counter's initial value, M, has been loaded, the counter proceeds to decrement.

On reaching zero, the $\mathrm{M}$-counter generates the load signal, $l d$, which causes the MMD to return to its initial state, where both counters are reloaded and $d i v_{p}$ reset to zero. During 
counting, the M-counter's changing output states are decoded to produce timing signals needed by other parts of the FD. The schematic for the M-counter is shown in Fig.4.11.

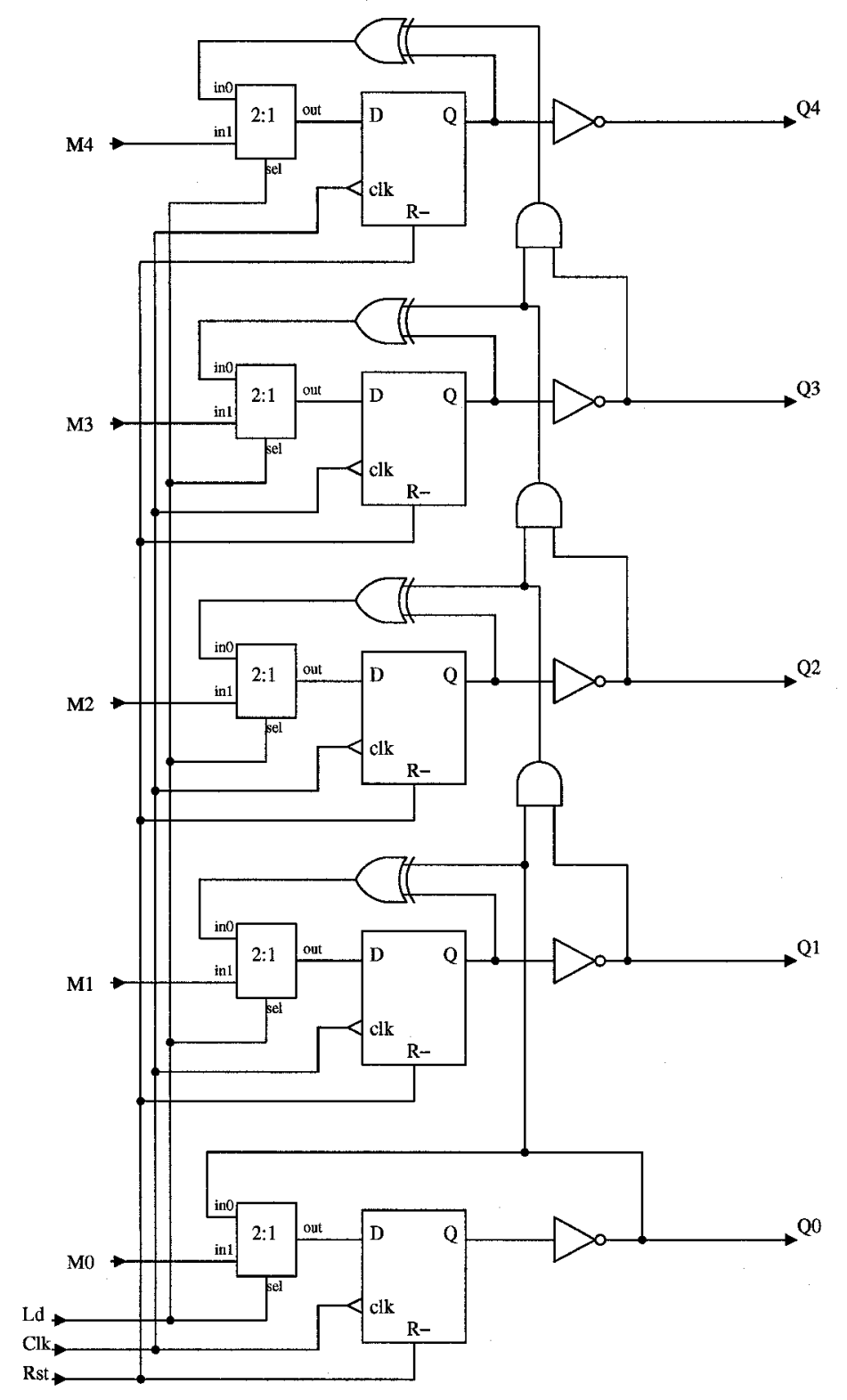

Figure 4.11: Schematic for M-Counter 


\subsubsection{A-Counter}

After the initial value of $\mathrm{A}$ is loaded, this counter decrements to zero. When zero $\left(Q_{1} Q_{0}={ }^{\prime} 00^{\prime}\right)$ is reached, $d i v_{p}$ goes high. The counter is 'frozen' in this last state until $l d$ is activated at the end of the state sequence. The schematic for the A-counter is shown in Fig.4.12.

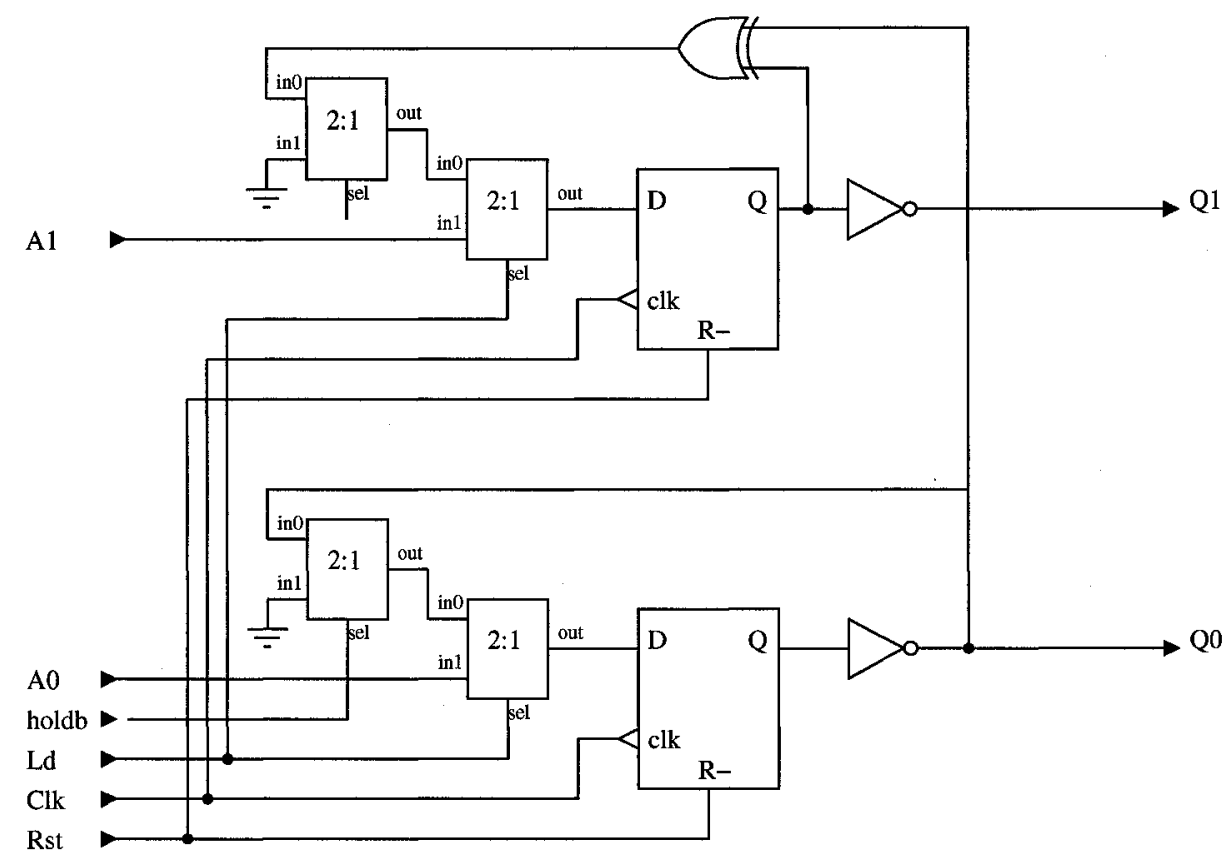

Figure 4.12: Schematic for A-Counter

\subsubsection{Decoding of M-Counter States}

As mentioned previously, timing signals needed by other FD components are derived from the M-counter's output states.

There are four outputs from the M-counter decoding circuit:

- $f_{d i v}$ : input to the PFD for phase comparison with $f_{\text {ref }}$. 
- $Q u a n_{c l k}$ : clock signal to the Quantizer.

- Digblk $c l k$ : clock signal to the Digital Feedback Block.

- $l d$ : load signal to initialize the MMD and reload the M-counter and the A-counter.

The timing relationships between those outputs are as follows:

Even when the loop is locked, $f_{\text {ref }}$ leads $f_{d i v}$ by a fixed time period because of the way PFD is designed, which is to be described in the next section. As a result, $P U$ and $P D$ have nearly equal durations when the loop is locked. The integrator converts the net transferred charge to a voltage which is sampled by the 3-bit quantizer after a settling time. There is , therefore, a time gap between the divider output $f_{d i v}$ and the clock $Q u a n_{c l k}$ that triggers the sampling.

The 3-bit digitized signal is fed to the Digital Feedback Block which uses it to generate a new divisor in time for the next cycle. The clock Digblk $k_{c l k}$ to the Digital Feedback Block must be delayed somewhat so that the quantizer data will be the most-recent.

The relationships between these outputs is illustrated in Fig.4.13.

Table.4.2 shows the relation between M-counter states and the four timing signals generated.

\begin{tabular}{|c|c|}
\hline Decoder Output & Corresponding States \\
\hline$f_{d i v}$ & $\overline{Q_{4}} Q_{3} Q_{2} \overline{Q_{1}} \bar{Q}_{0}$ \\
\hline$f_{q u a n}$ & $Q_{4} Q_{3} Q_{2} \overline{Q_{1}} \overline{Q_{0}}$ \\
\hline$f_{f d b}$ & $Q_{4} Q_{3} Q_{2} \bar{Q}_{1} Q_{0}$ \\
\hline$l d$ & $Q_{4} Q_{3} Q_{2} Q_{1} \overline{Q_{0}}$ \\
\hline
\end{tabular}

Table 4.2: Decoder Outputs 


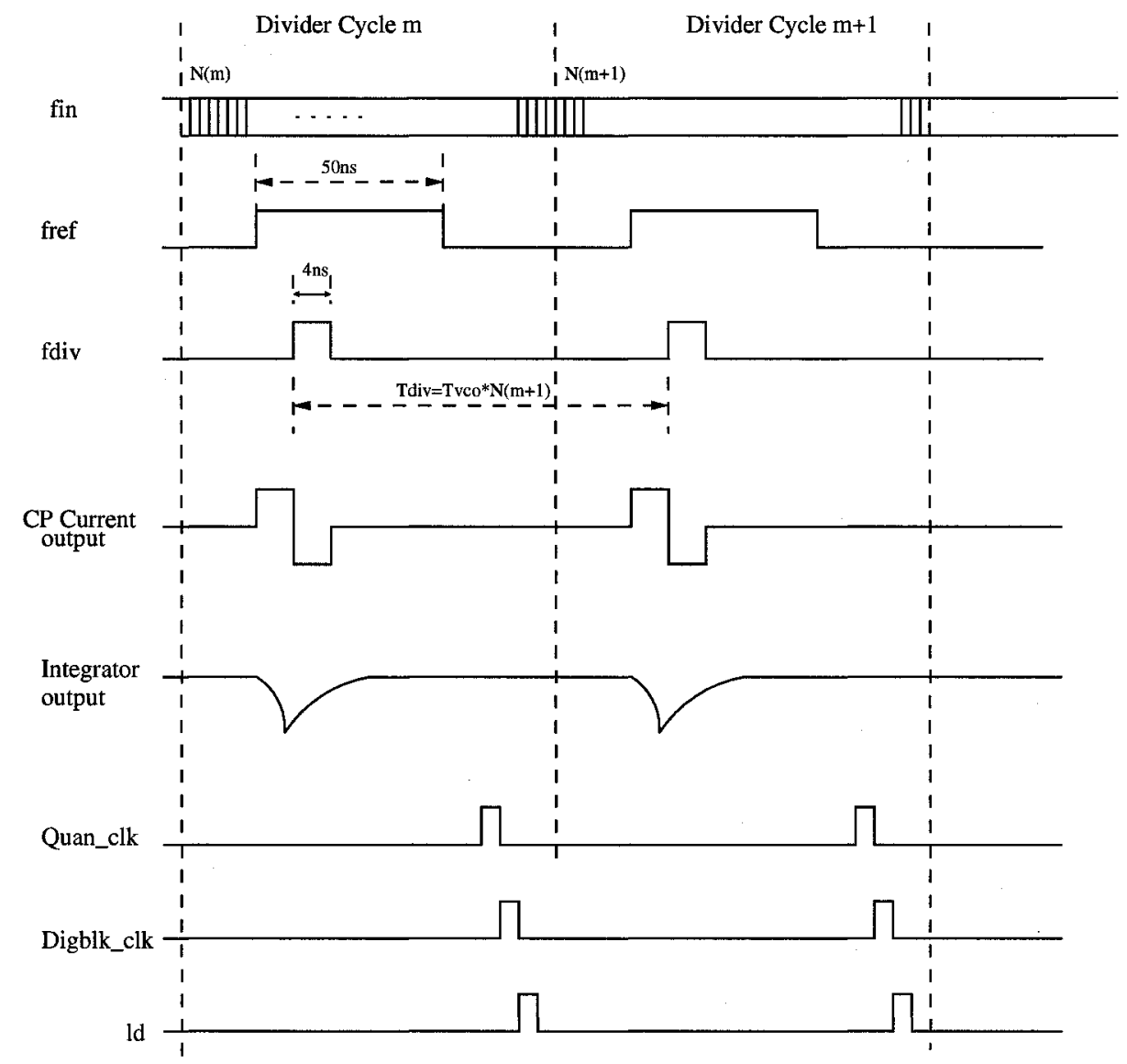

Figure 4.13: Mapping of M-counter States to MMD Timing Signals 


\subsubsection{Simulation of MMD}

RTL simulation results for the MMD are shown in Fig.4.14.

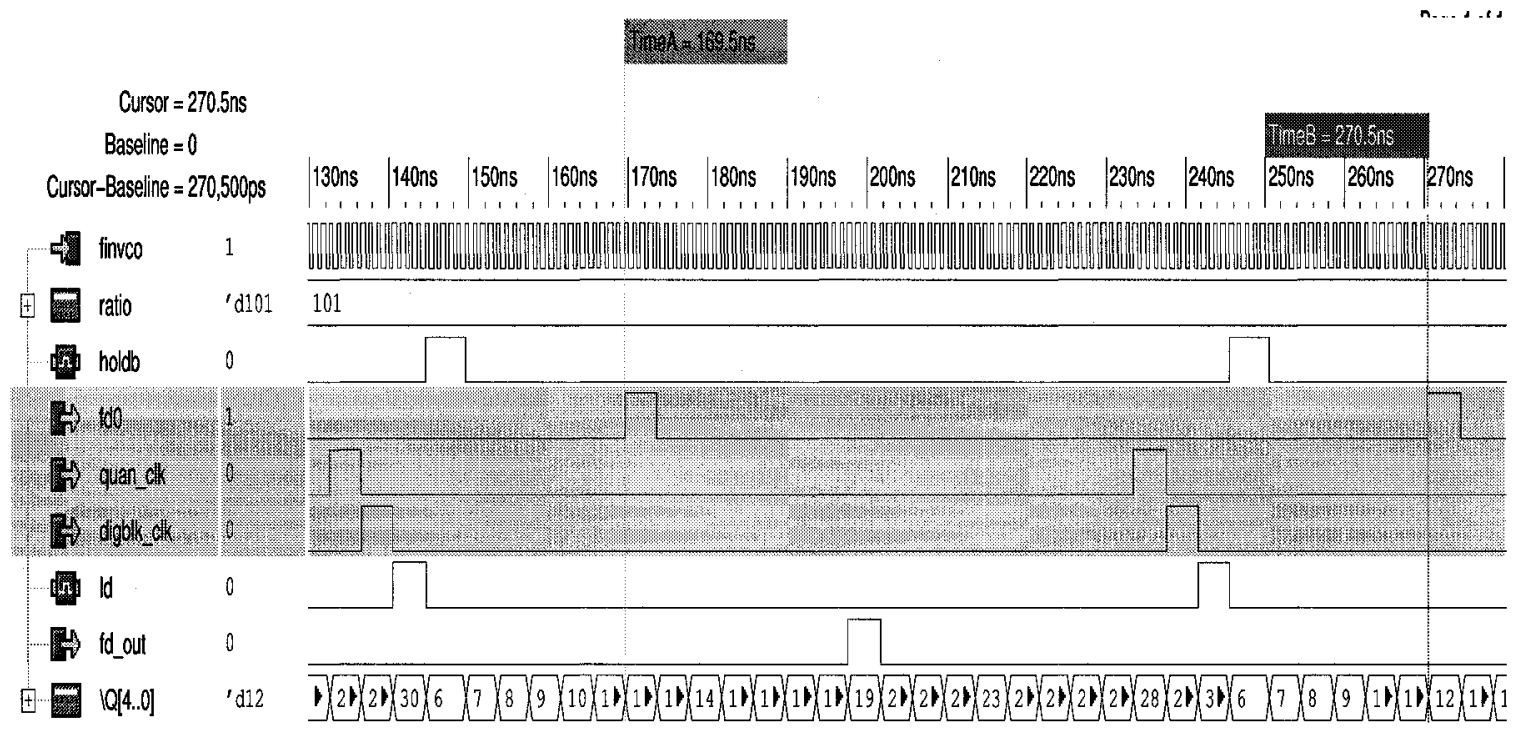

Figure 4.14: RTL Test Bench Simulation Results

In the simulation, the divisor components $M$ and $A$ are encoded as $M_{4} M_{3} M_{3} M_{2} M_{1} M_{0} A_{1} A_{0}=$ 1100101 (decimal 101), which means that the M-counter will decrement from $11001\left(d^{\prime} 25\right)$ to 00001 . The load signal $l d$ is active in the last state (00001). The active $l d$ causes the M-counter and A-counter to be re-loaded and then $d_{i} v_{p}$ to be set high. $f_{\text {in }}$ is divided by $P+1=5$ once $\left(A_{1} A_{0}=01\right.$ times $)$, and then by $P=4$ twenty-five times $(M-A)$ when $\operatorname{div}_{p}$ is set low.

The $f_{d i v}, Q u a n_{c l k}$ and Digblk $k_{c l k}$ signals are derived from the M-counter's output states as described in Table.4.2. 


\subsection{Phase-Frequency Detector}

The PFD converts the phase error to pump-up and pump-down pulse signals ( $P U$ and $P D$ ), the difference in widths of these being proportional to the phase error. The difference is positive when $f_{\text {ref }}$ leads $f_{d i v}$ and negative when $f_{\text {ref }}$ lags $f_{d i v}$. The detector is such that a phase error of $+2 \pi$ occurs when the $P U$ pulse width is $T_{r e f}$ and the $P D$ width is zero, so the PFD gain is $K_{P F D}=\frac{T_{r e f}}{2 \pi}$.

The schematic for the PFD is shown in Fig.4.15.

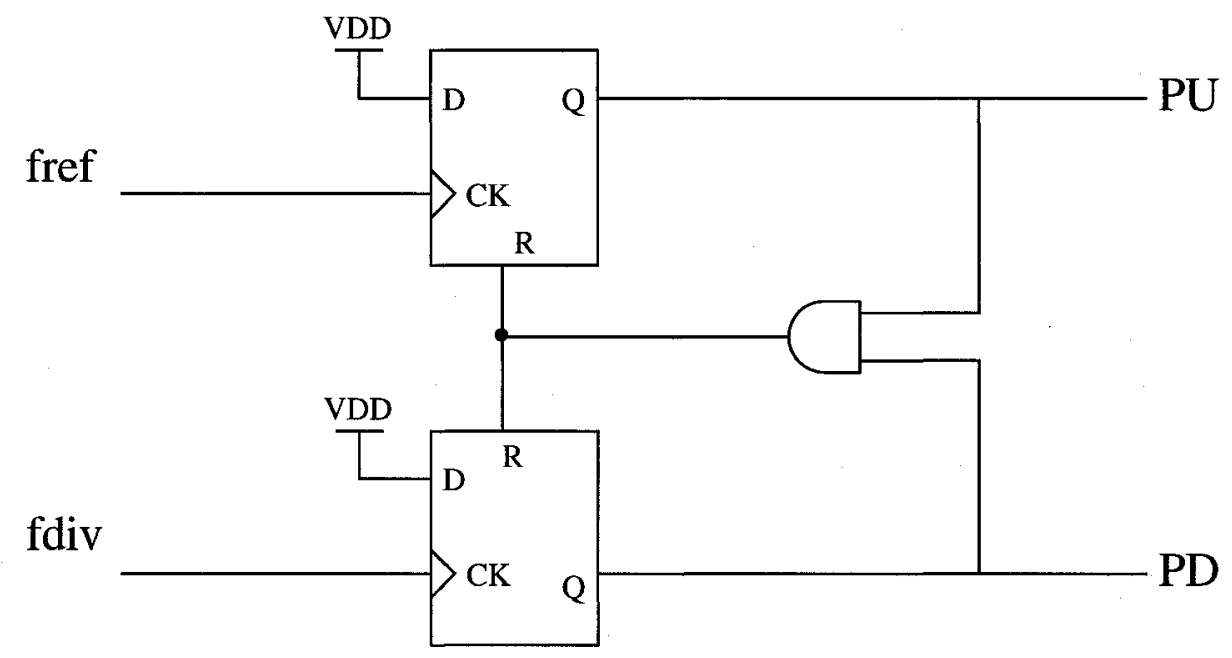

Figure 4.15: A schematic for a PFD

The circuit functions as follows:

If $f_{\text {ref }}$ leads $f_{\text {div }}$, the rising edge of $f_{\text {ref }}$ sets $P U$ high, where it remains until the rising edge of $f_{\text {div }}$ arrives and sets $P D$ high. $P U$ and $P D$ are logically 'and'ed to form a short reset pulse to clear both outputs. The width of the $P U$ pulse is proportional to the phase difference between $f_{\text {ref }}$ and $f_{\text {div }}$.

If $f_{\text {div }}$ leads $f_{r e f}$, the rising edge of $f_{d i v}$ sets $P D$ high, where it remains until the rising edge of $f_{\text {ref }}$ arrives and sets $P U$ high. $P U$ and $P D$ are logically 'and'ed to form a short 
reset pulse to clear both outputs. The width of the $P D$ pulse is proportional to the phase difference between $f_{d i v}$ and $f_{r e f}$.

The above circuit has a dead-zone problem when the widths of the $P U$ and the $P D$ pulses are comparable to their transition rising/falling times, as may happen when the phase error is close to zero. A solution to this problem, which also improves PFD linearity, is to introduce a fixed DC phase offset to move the lock point away from the zero phase error point, as illustrated in Fig.4.16.

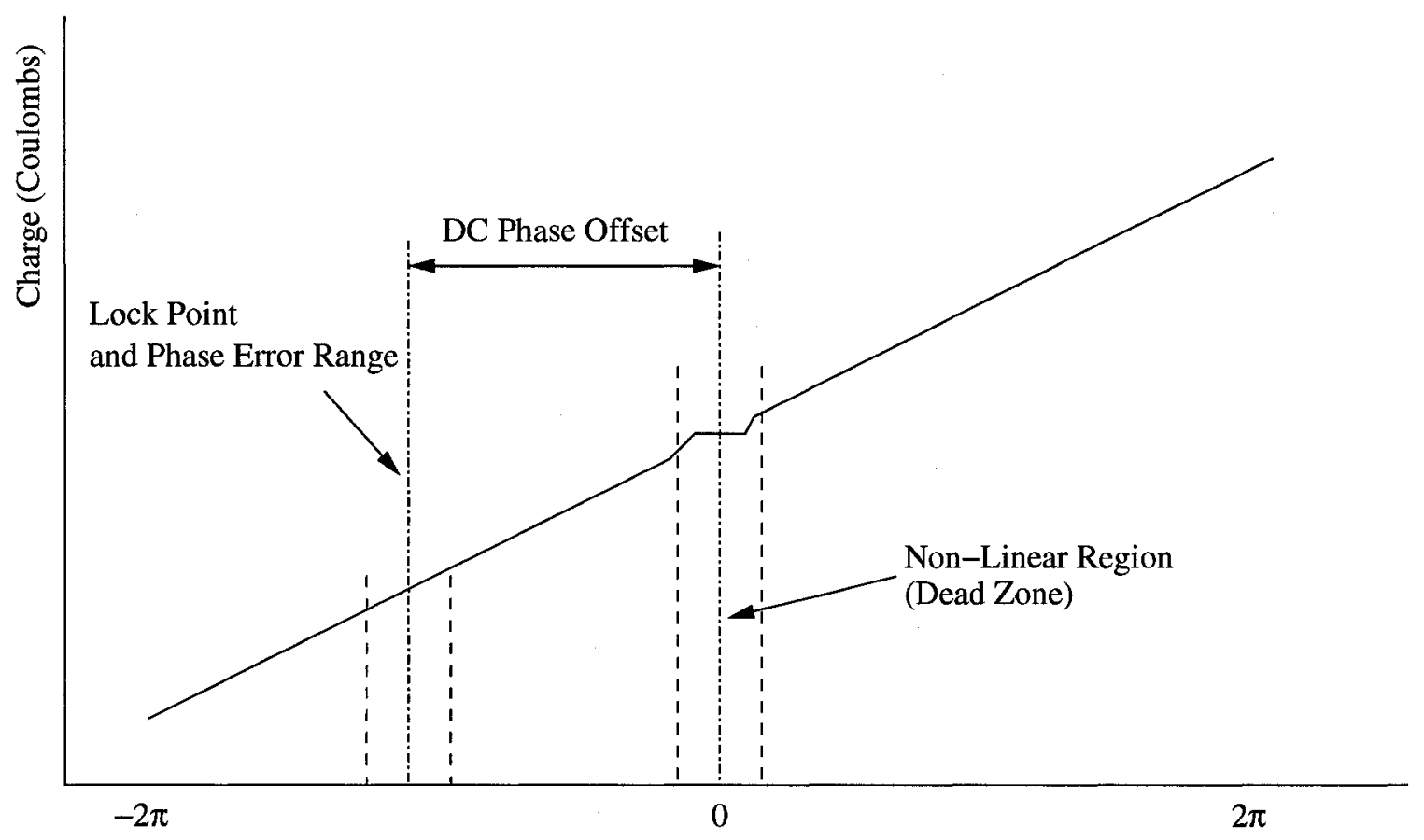

Phase Error (Radians)

Figure 4.16: PFD with DC offset

When the loop is locked, $f_{\text {ref }}$ will always lead $f_{\text {div }}$ for a fixed time period, thus establishing a minimum $P U$ pulse width. The circuit is designed to emit a $P D$ pulse of exactly the same length. The charge transferred to the integrator during the minimum $P U$ time is 
removed during the $P D$ time and the net effect on the integrator output (the error voltage) will be zero. The loop will remain locked in spite of the introduction of the phase offset.

The downside of this approach is that since the duty cycle of $P U$ and $P D$ is no longer zero at the lock point, the charge pump will always operate for a minimum time equal to double the $P D$ pulse width in each $f_{\text {ref }}$ cycle. The system noise contribution will increase. The reference feed-through will also increase.

Fig.4.17 and Fig.4.18 show simulations of the PFD with offset in the cases where $f_{\text {ref }}$ leads and lags $f_{\text {div }}$ :

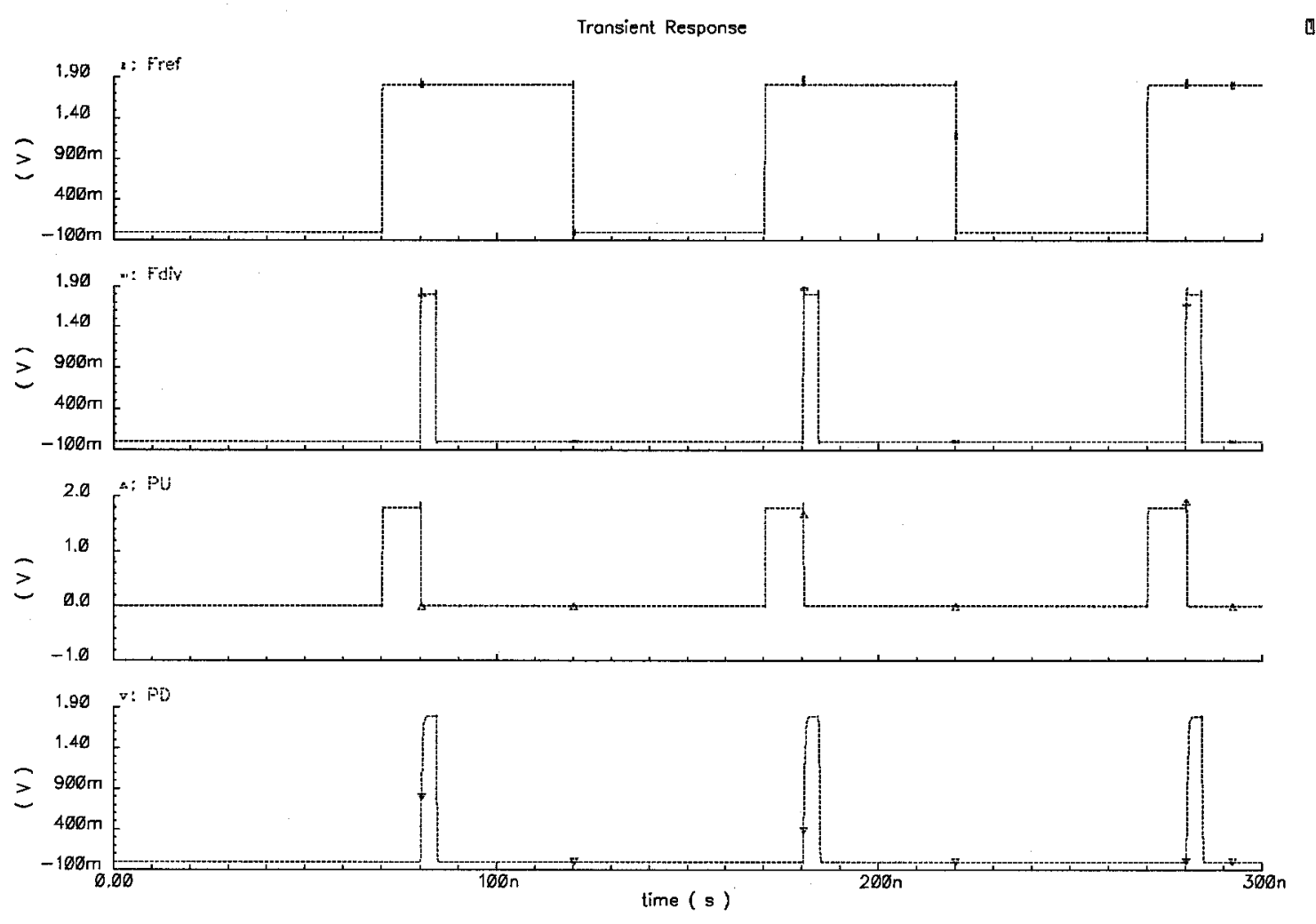

Figure 4.17: PFD Transition Simulation: Fref Leading Fdiv 


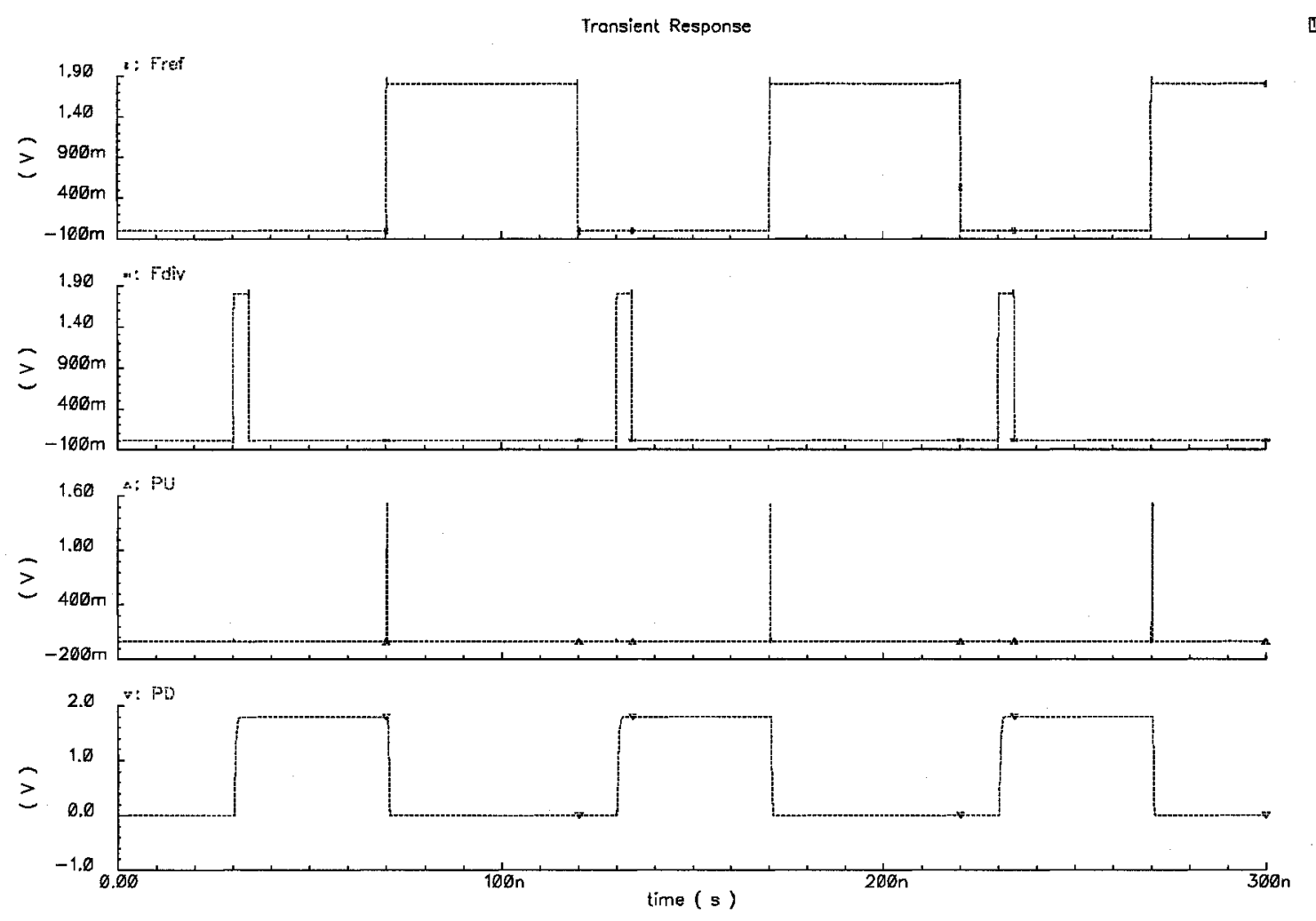

Figure 4.18: PFD Transition Simulation: Fref Lagging Fdiv

There are eight such PFDs used in this design, forming an array of eight PFDs. This array of eight PFDs is a part of the truncation error suppression scheme used in the new architecture for the fractional-N divider, as described in Chapter 3.

\subsubsection{PFD Noise Performance}

The PFD noise will be analyzed together with the CP noise as described in the next section. 


\subsection{Charge Pump}

The Charge Pump (CP) converts the difference between the $P U$ and the $P D$ pulse widths to an average current output during one $f_{r e f}$ cycle. If the widths are $t_{P U}$ and $t_{P D}$, the average current will be $I=\frac{I_{p}}{T_{r e f}}\left(t_{P U}-t_{P D}\right)$. The gain of the CP can be given by:

$$
K_{C P}=\frac{I_{p}}{T_{r e f}}
$$

Where $I_{p}$ is the constant current flow during a pump up or a pump down interval.

The schematic for one CP used in this design is shown in Fig.4.19.

The CP has three sections:

- Band Gap: provides an accurate current reference that is robust with regard to process, voltage and temperature variations.

- Mirror : forces source and sink currents to be equal and equal-to the band gap reference current.

- Charge Pump Element : generates a source or a sink current depending on state of $P U$ or $P D$ signals. 


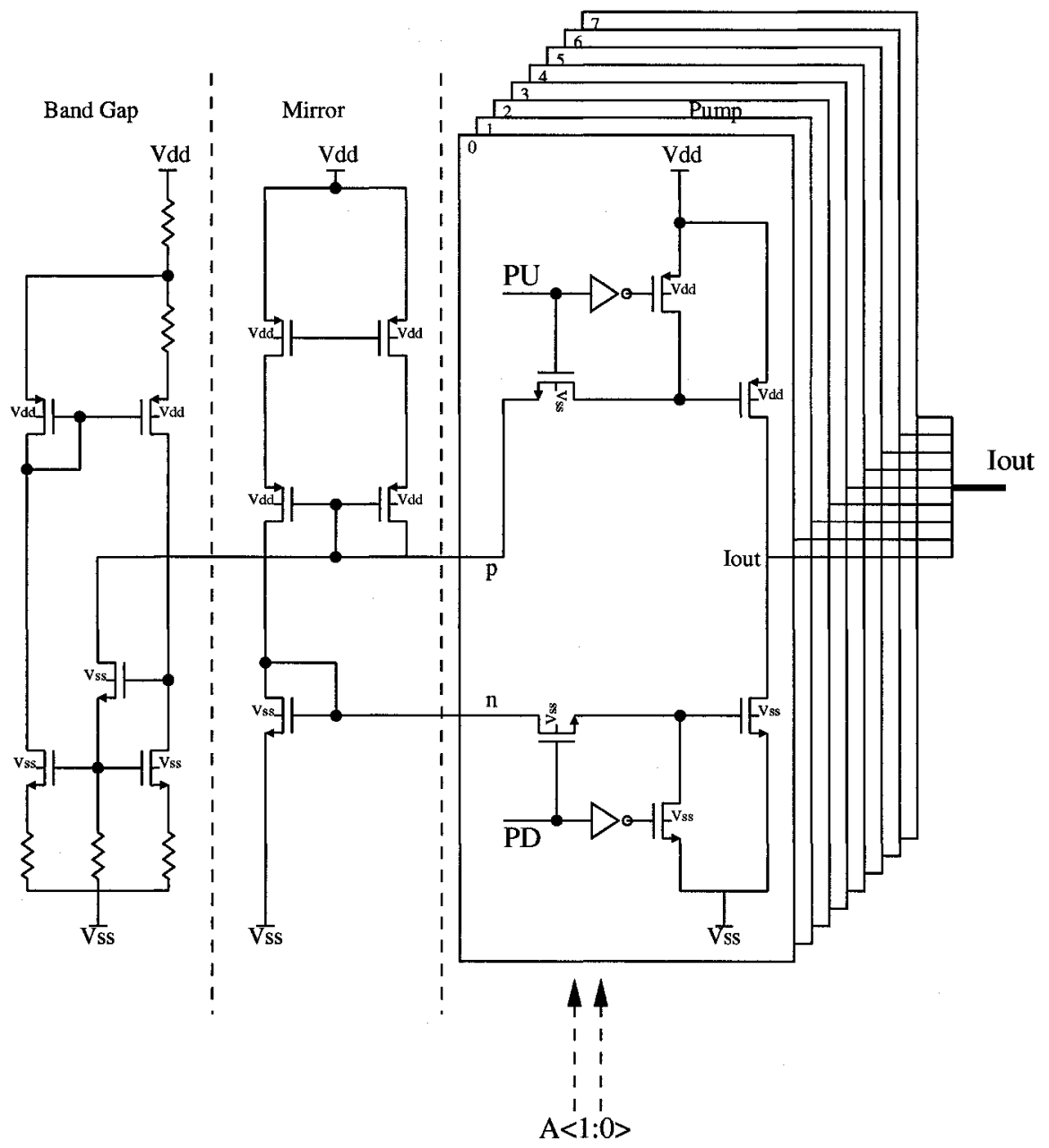

Figure 4.19: Schematic for one Charge Pump element

The CP output current is programmable by enabling or disabling parallel charge pump elements using two control bits, $A_{1}$ and $A_{0}$, as shown in Table.4.3. Control over the CP output current allows the designer a way of adjusting the system loop gain. 


\begin{tabular}{|c|c|c|c|}
\hline Control Bits & \# of CP element enabled & CP Output Current $(\mu A)$ & Array Output $(\mu A)$ \\
\hline$A_{1} A_{0}=11$ & 8 & 25 & 200 \\
\hline$A_{1} A_{0}=10$ & 7 & 21.875 & 175 \\
\hline$A_{1} A_{0}=01$ & 6 & 18.75 & 150 \\
\hline$A_{1} A_{0}=00$ & 5 & 15.625 & 125 \\
\hline
\end{tabular}

Table 4.3: CP Output Current vs Control Bits

There are eight such CPs used in this design, forming an array of eight CPs. This array of eight CPs is a part of the truncation error suppression scheme used in the new architecture for the fractional- $\mathrm{N}$ divider, as described in Chapter 3.

The maximum output current of the array, which is $200 \mu \mathrm{A}$, is the sum of the maximum output of each CP. The behaviour of the array of eight CPs at its maximum current output has been simulated. The result is shown in Fig.4.20. 


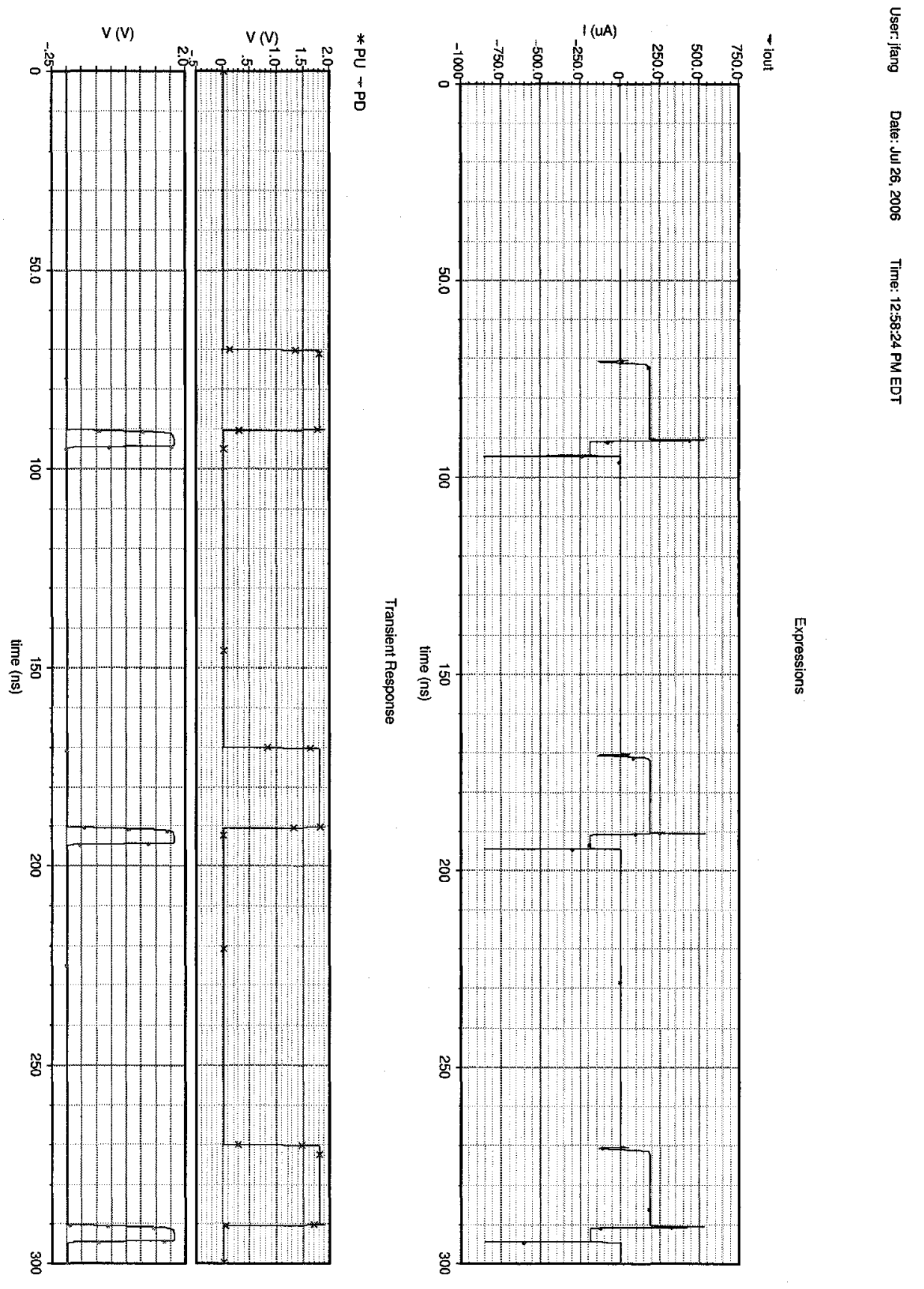

Figure 4.20: Charge Pump Operation 


\subsubsection{PFD/CP Noise Performance}

PFD and CP noise performance was simulated using the Spectre PSS and Pnoise packages. This simulation was done with an array of eight CPs and their associated PFDs. The eight PFDs are supplied with the same input. The total output current is $200 \mu \mathrm{A}$. The output-referred noise, in units of amperes $/ \sqrt{H z}$, is shown in Fig.4.21.

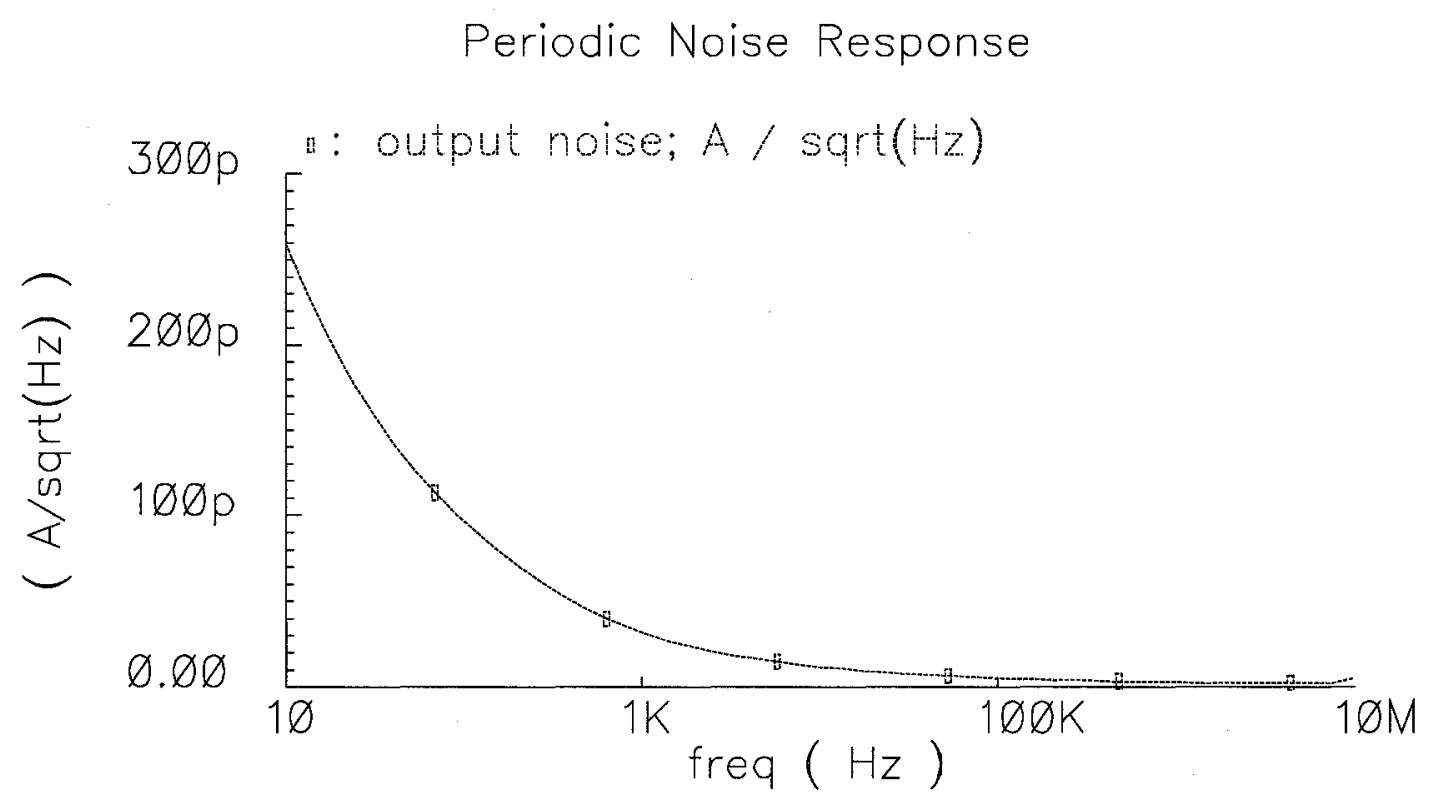

Figure 4.21: PFD/CP Output-Referred Noise 


\subsection{Integrator}

The integrator is a current to voltage converter which transforms $\mathrm{CP}$ array output current to a voltage which can be sampled by the Quantizer. An integrator may be passive or active. From the system analysis point of view, there is no difference between the two, but the choice of one over the other is significant for the circuit design.

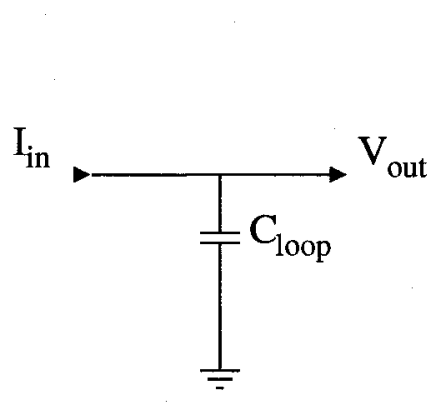

(a)

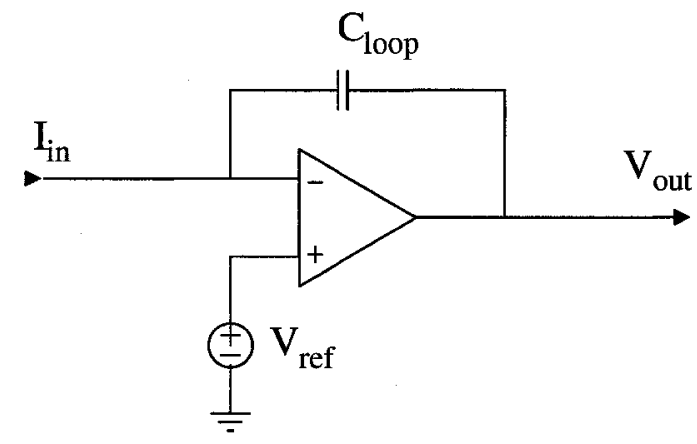

(b)

Figure 4.22: (a) a Passive Integrator and (b) an Active Integrator

If a passive integrator, as shown in part a of Fig.4.22, were used, the requirement of voltage compliance would make $\mathrm{CP}$ design more challenging. That is, a $\mathrm{CP}$ feeding a passive integrator must be capable of producing a constant source or sink current over a wide output voltage range. This problem does not occur when an active integrator, as shown in part b of Fig.4.22, is used because the virtual ground at the $(+)$ input holds the $(-)$ input to $V_{r e f}$. Another benefit of using an active integrator is the reduction of kickback noise from the comparators used in the Quantizer [FV04]. 
The schematic for the active integrator chosen for this design is shown in Fig.4.23.

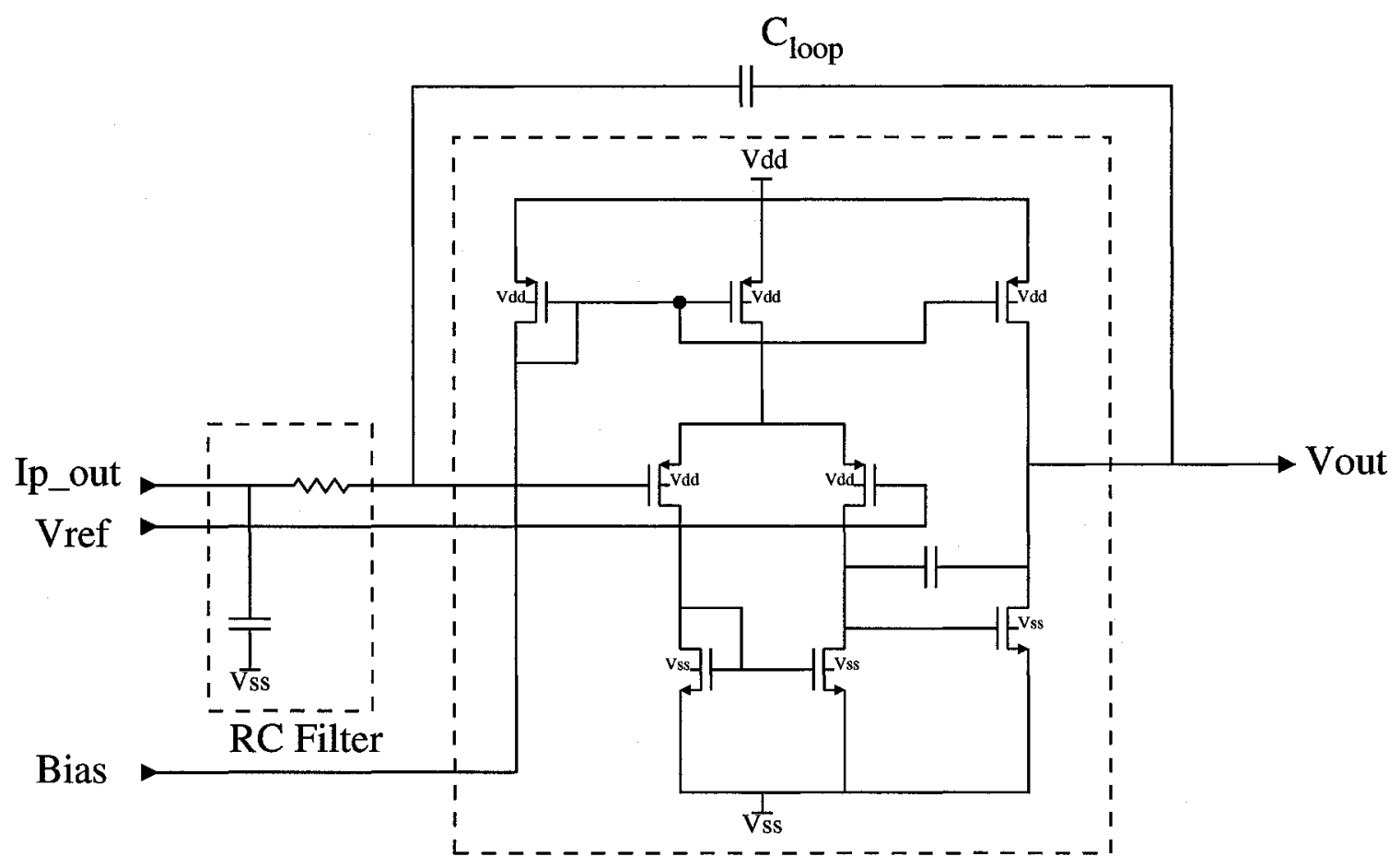

OP AMP

Figure 4.23: Schematic for a Active Integrator

The OP-AMP uses a differential input stage having an active load followed by a common source stage also driving an active load. It has a small number of transistors, high open loop gain, rail to rail output range and a large input range with only one frequency compensation capacitor being needed [HBL01].

The RC filter in the OP-AMP's input path eliminates the effects of op-amp slew rate limitations which could cause non-linear operation. 
The integrator's response to CP output pulses is shown in Fig.4.24.

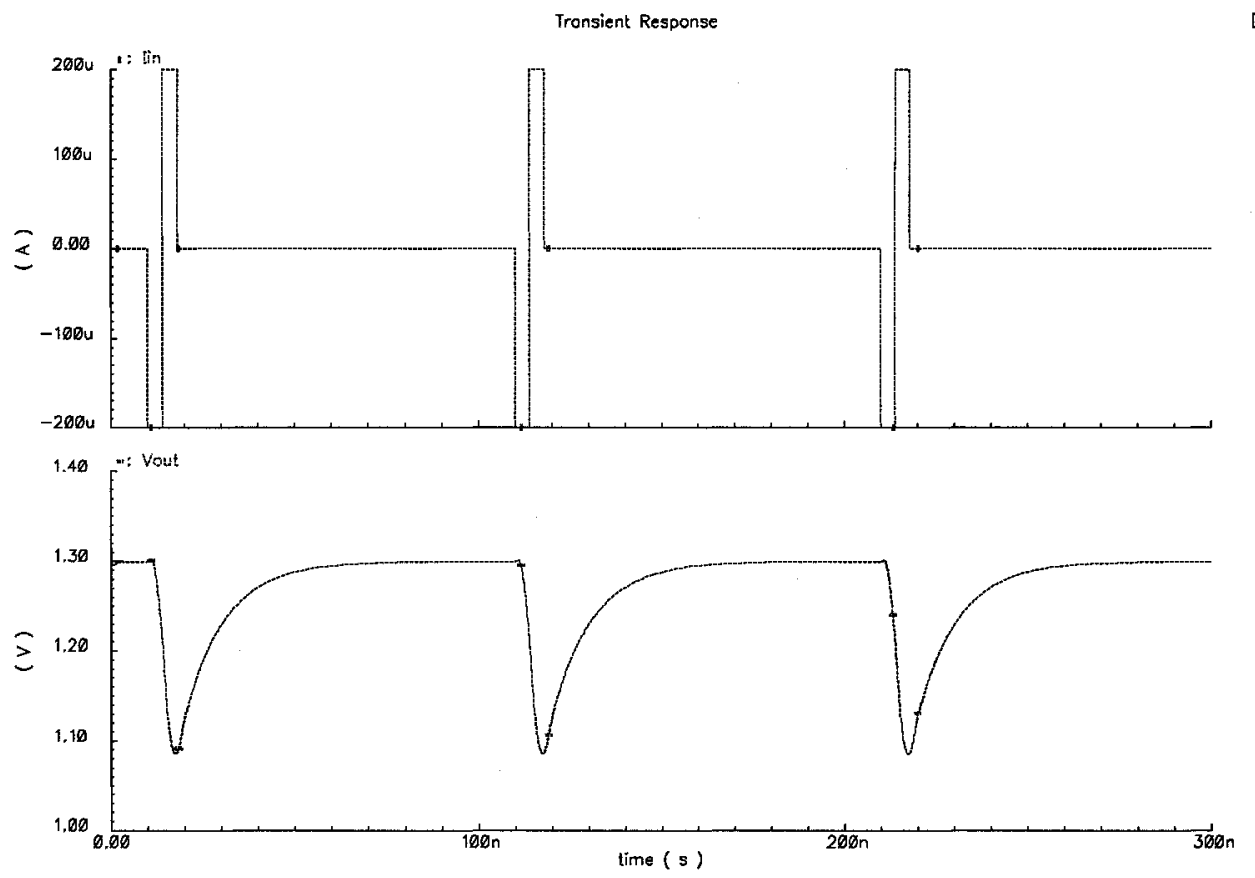

Figure 4.24: Integrator Transient Response 


\subsubsection{Noise Performance}

The integrator's noise performance was simulated using the Spectre PSS and Pnoise packages. The output-referred noise, in units of volts $/ \sqrt{(} \mathrm{Hz})$, is shown in Fig.4.25.

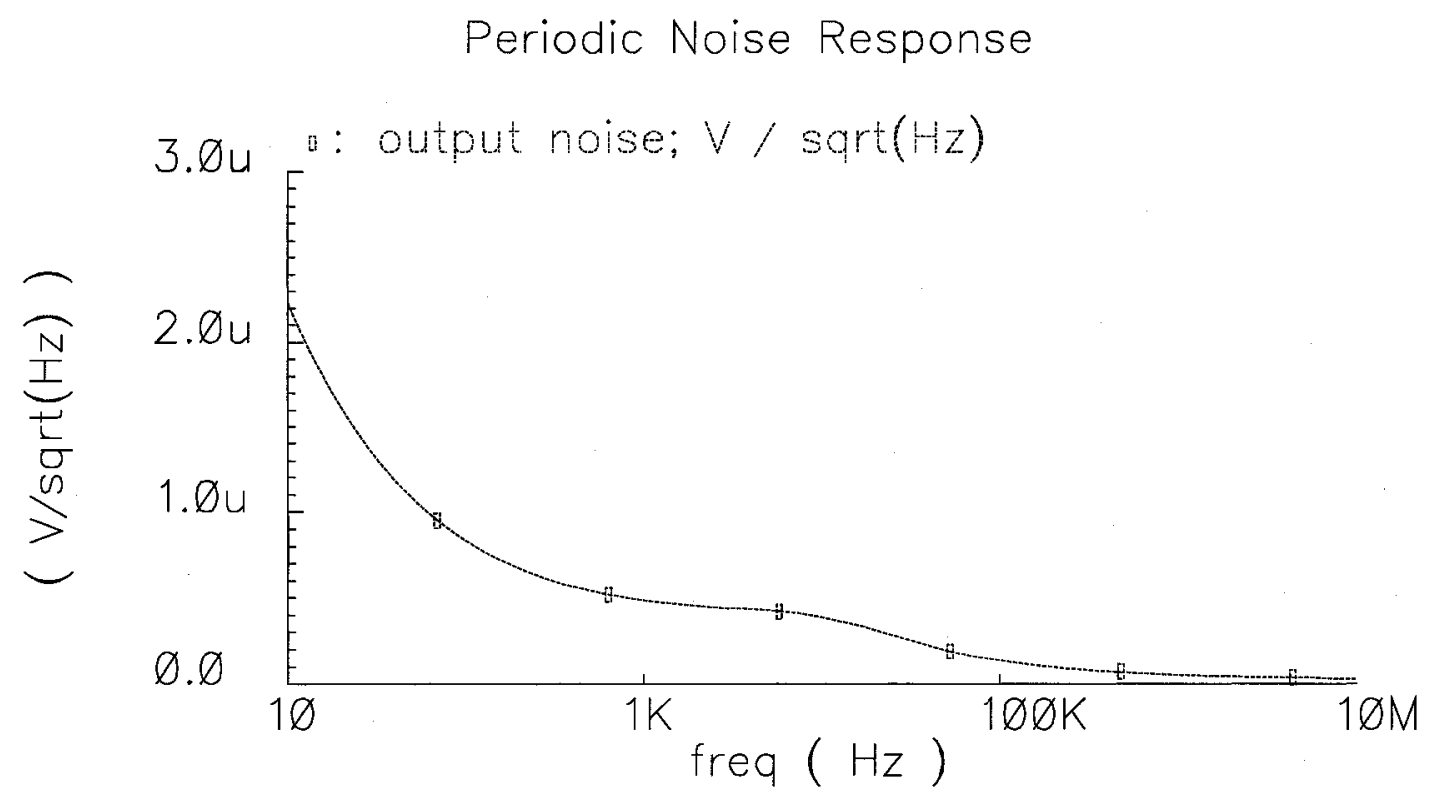

Figure 4.25: Integrator Output Referred Noise for Lock Condition 


\subsection{3-Bit Quantizer}

Quantizers are essentially A/D converters. In this thesis, an array of eight comparators, as shown in Fig.4.26, is used to implement a 3-bit quantizer.

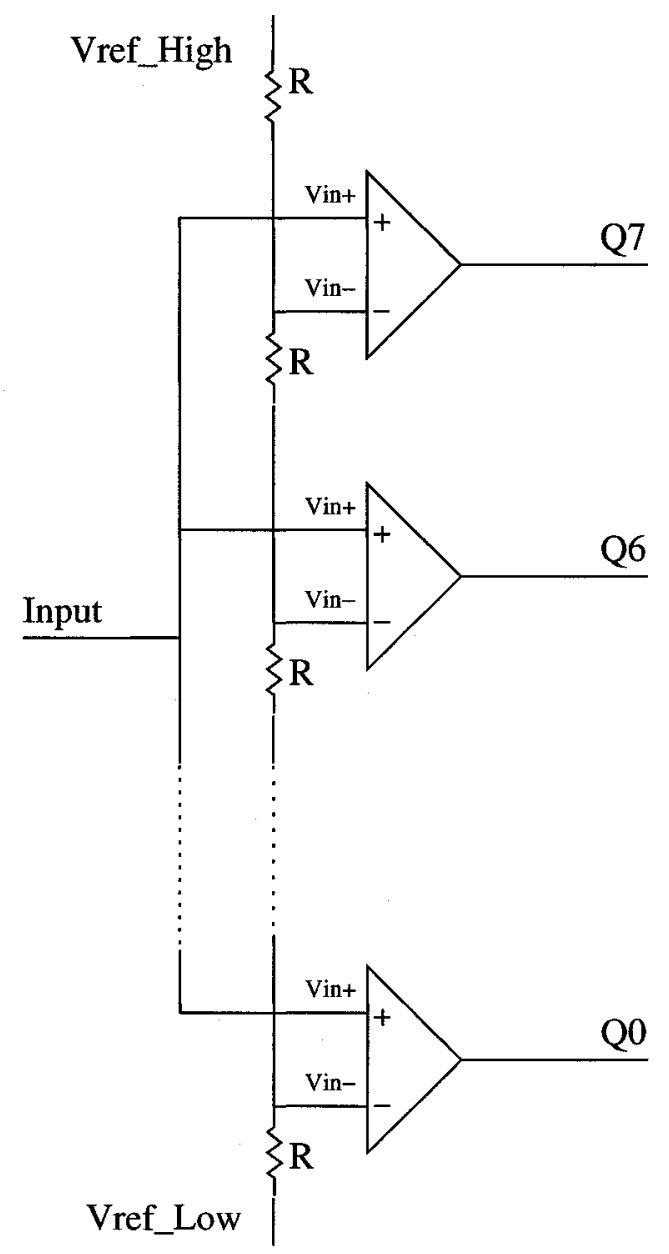

Figure 4.26: An Array of Eight Comparators Used as a 3-bit Quantizer 
The quantizer's input resolution, voltage step size $\Delta V$, must be the same as the voltage swing at the integrator's output caused by a $T_{i n} / 8$ phase error at the input of the CP. In this case, $\Delta V$ is given by:

$$
\Delta V=\frac{T_{\text {in }}}{8} \frac{I_{\text {pump }}}{C_{\text {loop }}}
$$

Where $T_{\text {in }}=1 n s, I_{\text {pump }}=200 \mu A$ and $C_{\text {loop }}=1 p F$. ( $C_{\text {loop }}$ is the capacitor used in the integrator, as shown in Fig.4.23.) Thus:

$$
\Delta V=\frac{1 n s}{8} \frac{200 \mu A}{1 p F}=25 m V
$$

The total range of the quantizer is therefore $V_{\text {ref_high }}-V_{\text {ref_low }}=8 \Delta V=200 \mathrm{mV}$.

When the loop is locked, the PFD's DC offset will cause the quantizer's input voltage to first decrease and then to increase by an amount given by:

$$
V_{\text {offset }}=T_{\text {offset }} I_{\text {pump }} / C_{\text {loop }}=\frac{4 n s \times 200 \mu A}{1 p F}=800 \mathrm{mV}
$$

As an RC filter is used in the integrator's input path, the voltage decrease/increase is reduced to somewhere near $200 \mathrm{mV}$, as shown in Fig.4.24.

For testing purposes, external pins are available for adjusting $V_{\text {ref_high }}$ and $V_{\text {ref_low }}$.

\subsubsection{Comparator Design}

The schematic for the comparator is shown in Fig.4.27. 


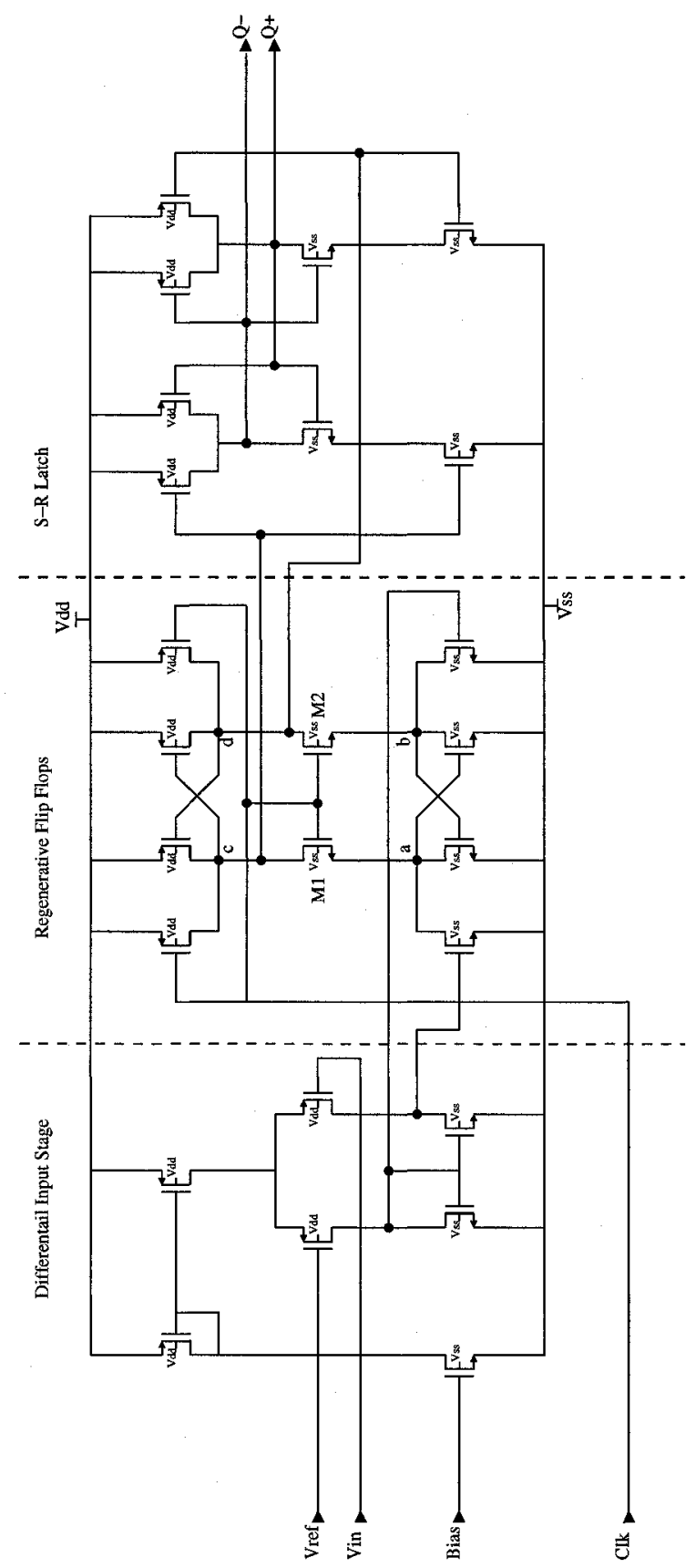

Figure 4.27: Comparator Schematic 
The comparator has a differential input stage followed by two regenerative flip-flops and an S-R latch [YES92] [Yuk85].

A cycle of operation of this circuit consists of a settling period followed by a regeneration period:

\section{Settling Period:}

This is the period when the clock is low. Switches $M 1$ and $M 2$ are off, the n-channel flip-flops disconnected from the p-channel flip-flops, and a voltage proportional to the input voltage difference $\left(V_{i n}-V_{r e f}\right)$ is established between nodes $a$ and $b$. This voltage will act as the initial imbalance for the following regeneration period.

\section{Regeneration Period:}

When the clock goes high, switches $M 1$ and $M 2$ are turned on and the n- and pchannel flip-flops regenerate the voltage difference between nodes $a$ and $b$ and also between nodes $c$ and $d$. This latter voltage difference (between $c$ and $d$ ) is soon amplified to a voltage nearly equal to the power supply. The following S-R latch drives the complementary digital output levels at the end of the regeneration period.

\subsubsection{Simulation Results}

The integrator and quantizer combination was simulated. The simulation results are shown in Fig.4.28. The input current to the integrator simulates a phase error of $T_{i n} / 8$ by making the width of the sink current pulse $T_{i n} / 8$ longer than the source pulse. During each $f_{\text {ref }}$ cycle, the integrator output voltage is reduced by $\Delta V=25 \mathrm{mV}$, which is sampled by the quantizer at the end of the cycle. 

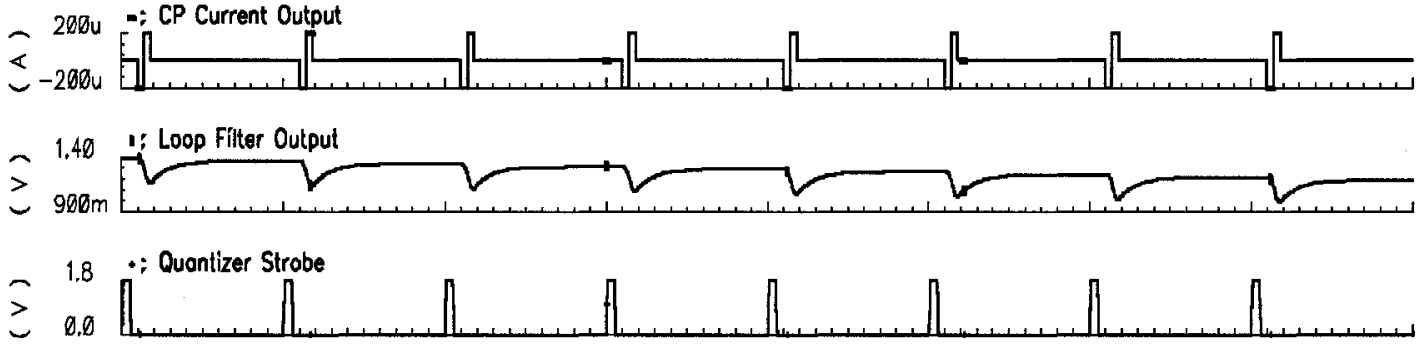

$\underset{>-160 \mathrm{~m}}{>1,90}$

$>1.90 \div 05$

-1.90 :04

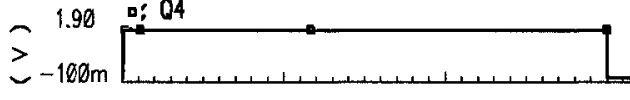

- $1.90 \because 03$

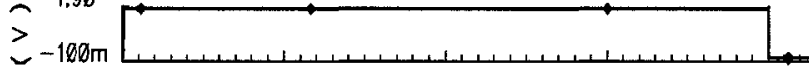

$1.90 \triangle 02$

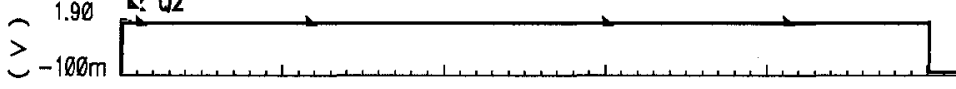

$-1.90 \div 01$
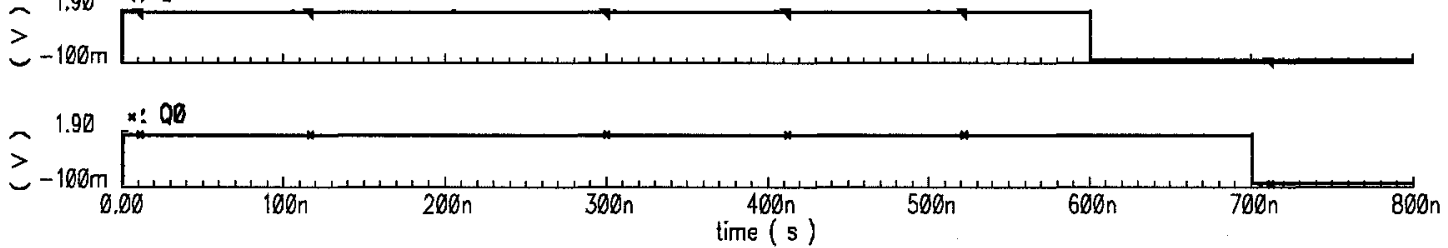

Figure 4.28: Quantizer Simulation 


\subsubsection{Noise Performance}

Fig.4.29 shows the results of the quantizer noise simulation. Since the quantizer is a non-linear device, the noise measurement is performed on the differential input stage only.

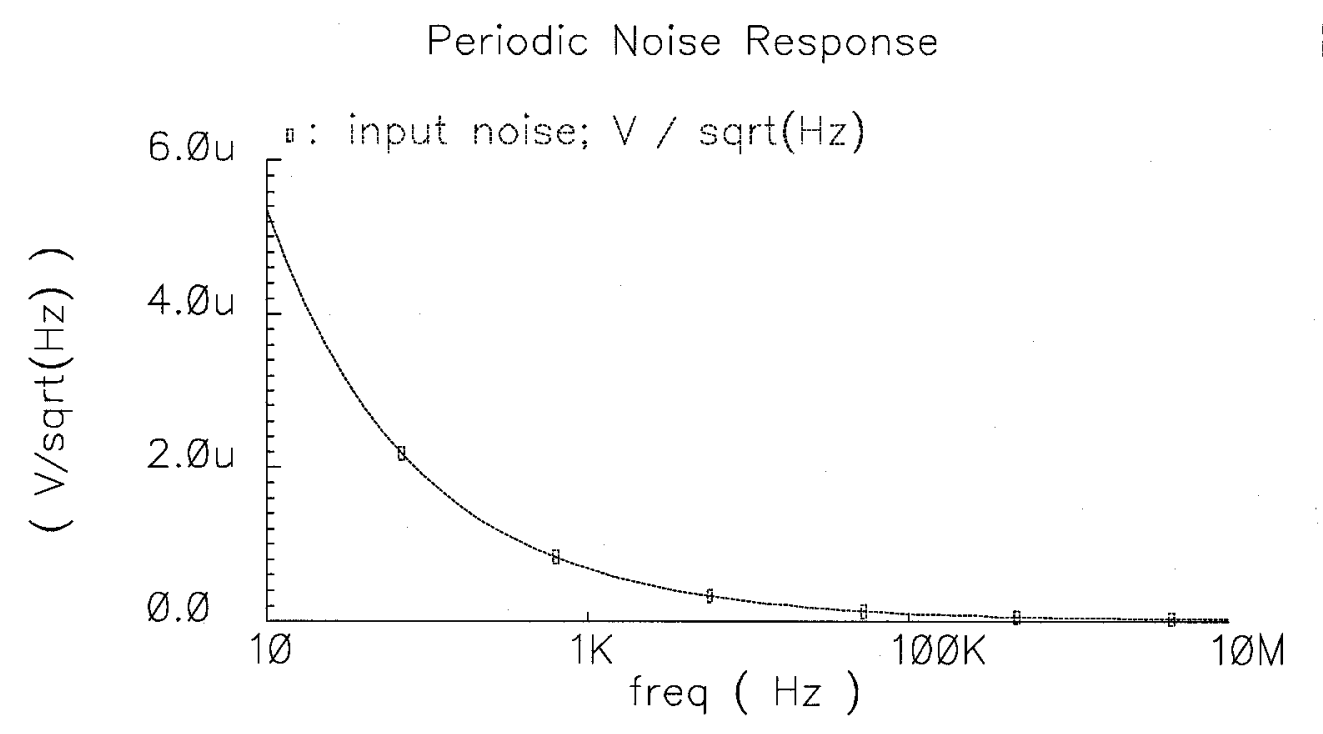

Figure 4.29: Quantizer Input-Referred Noise 


\subsection{Digital Feedback Block (DFB)}

The main function of the DFB is to process the quantizer output and generate the divisor for the divider in Fig.3.14. The all-digital nature of the DFB means that the design can be implemented using Hardware Description Language (HDL).

The DFB consists of five blocks. The block diagram is shown in Fig.4.30.

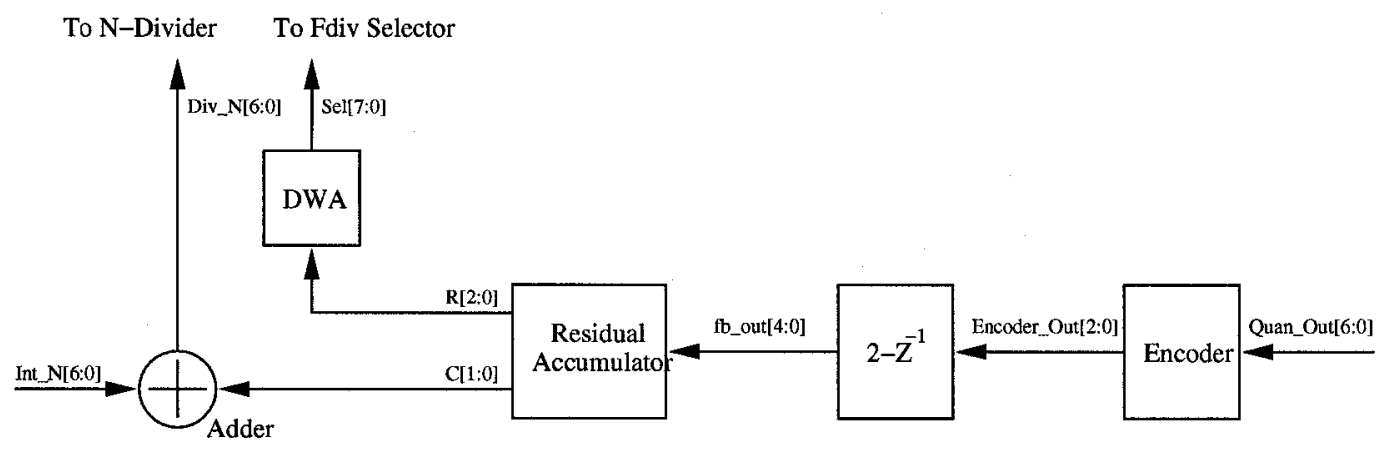

Figure 4.30: The Digital Feed Back Block

\subsubsection{Encoder}

This encodes the quantizer output into a 3-bit binary data.

\subsection{2 $2-Z^{-1}$ block}

Here, the operation $2 X_{n}-X_{n-1}$ is performed, where $X_{n}$ and $X_{n-1}$ are the current and previous Encoder outputs. Since the quantizer's output is in the range from 0 to 7 , the range of $2-Z^{-1}$ is from -7 to 14 . To avoid negative numbers, an offset of 8 is added. 


\subsubsection{Residual Accumulator}

Fig.4.31 presents the block diagram for the Residual Accumulator.

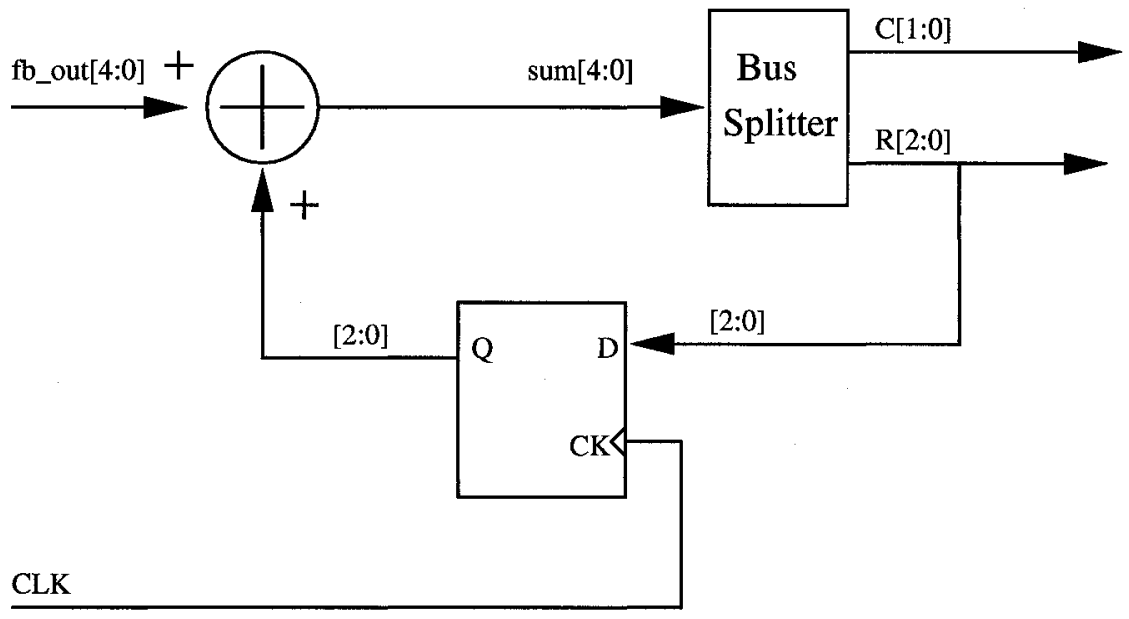

Figure 4.31: Residual Accumulator

\subsubsection{Data Weighted Averaging Block}

The circuit implementation of the DWA function, described in section 3.8 of Chapter 3, is shown in Fig.4.32. It performs the cyclic selection process as described in section 3.8. 


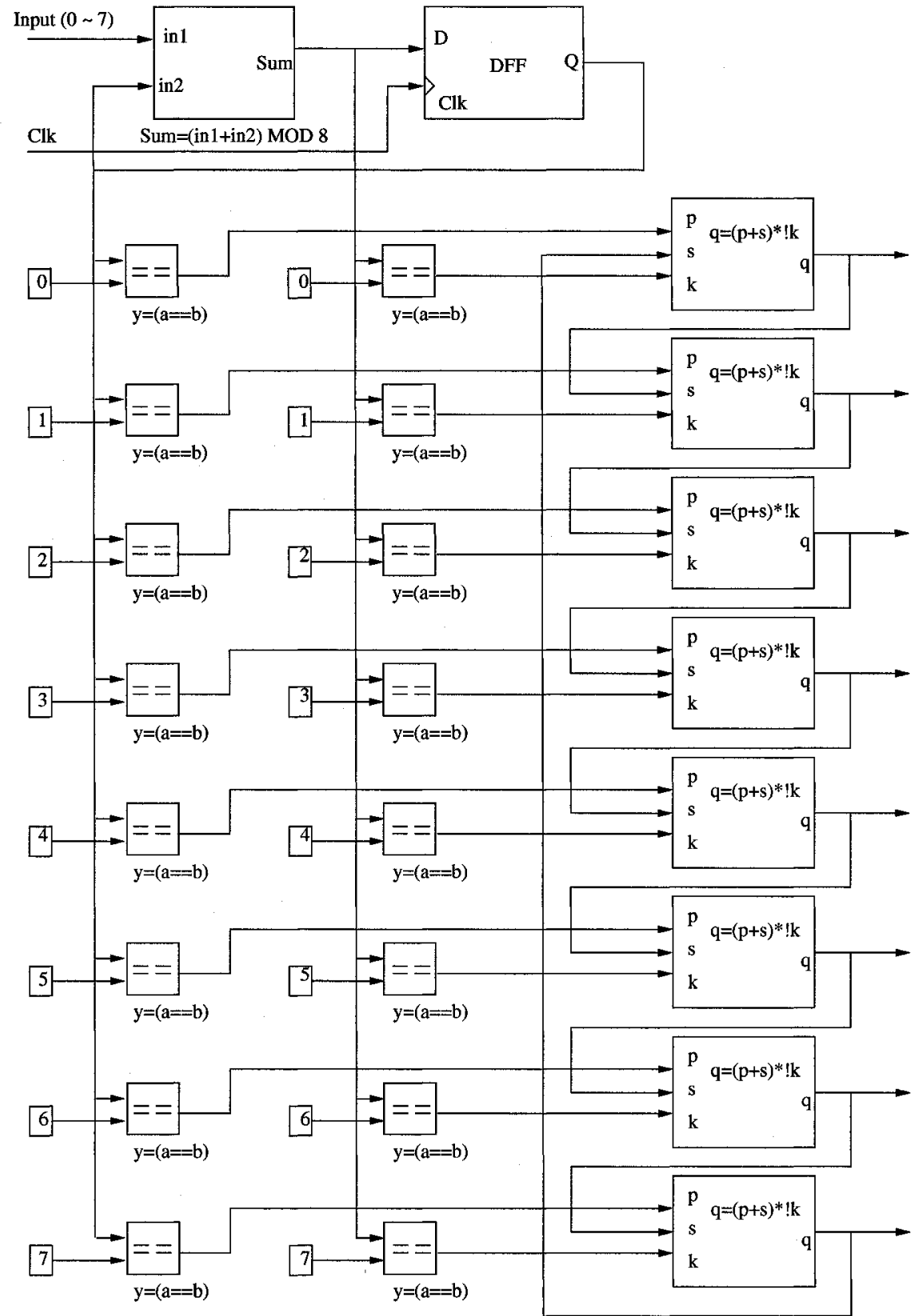

Figure 4.32: Block Diagram for DWA implementation 


\subsubsection{Simulation Result}

The RTL simulation result for the DFB is shown in Fig.4.33. Each row of the RTL simulation result corresponds to a node in the block diagram of Fig.4.30.

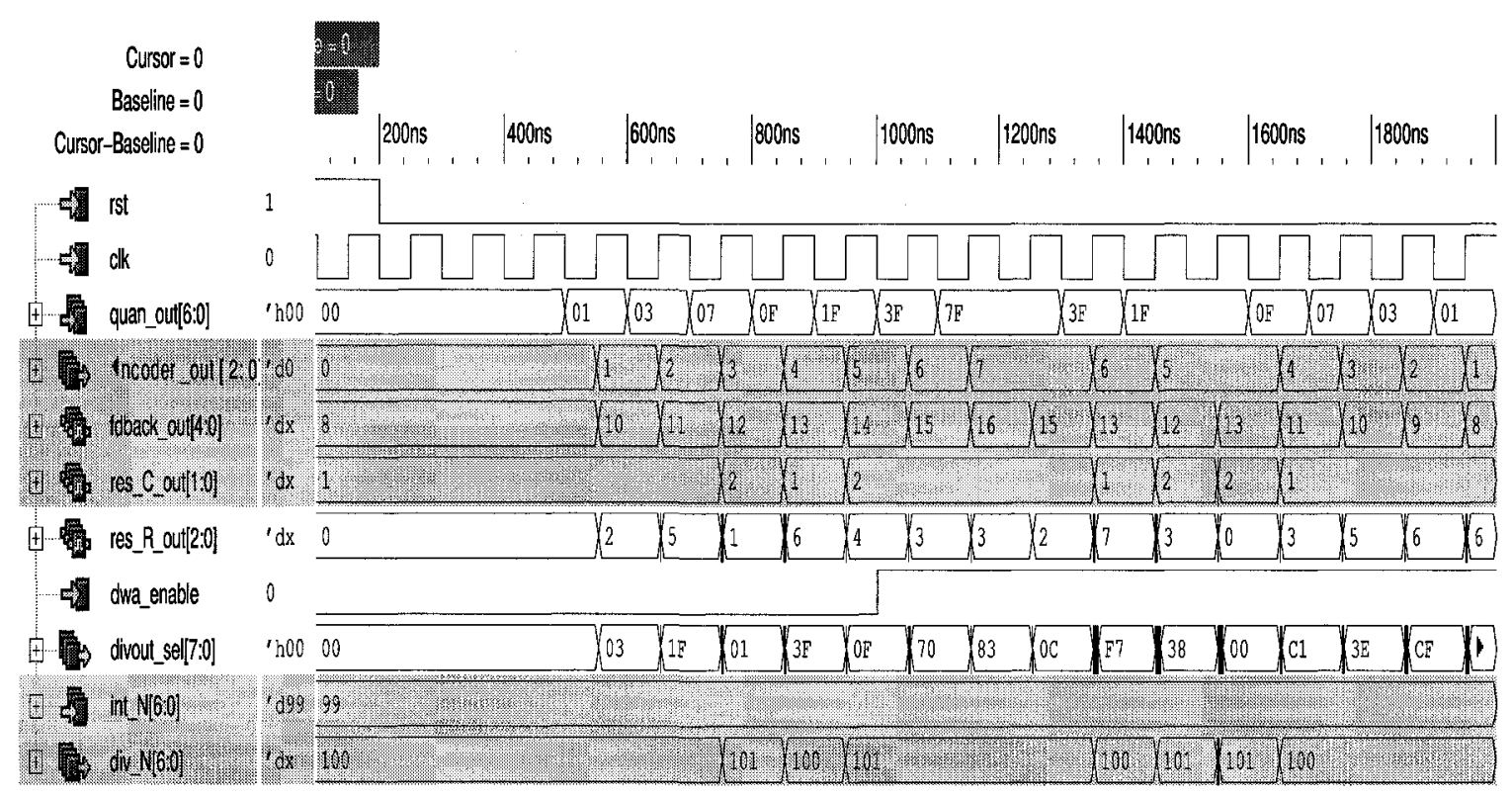

Figure 4.33: DFB RTL Simulation Result

In this simulation, for example at $600 \mathrm{~ns}$, when the Quantizer output changes from $h^{\prime} 01$ to $h^{\prime} 03$, the output of the Encoder changes from $d^{\prime} 1\left(X_{n-1}\right)$ to $d^{\prime} 2\left(X_{n}\right)$ (number of '1's in the Quantizer output). The $2-Z^{-1}$ block then produces $2 X_{n}-X_{n-1}+d^{\prime} 8=d^{\prime} 11$. The Accumulator splits $d^{\prime} 11$ into carry over $C=d^{\prime} 1$ and the residual $R=d^{\prime} 3$. $C$ is used to add on the base divisor int $N=d^{\prime} 99$ to generate the divisor $\operatorname{div} N=d^{\prime} 100$ for the next cycle. $R$ is used to determine the selection of $f_{d 0}$ or $f_{d 1}$, as shown in Fig.3.11. In this example, 3 $f_{d 0}$ and $5 f_{d 1}$ will be selected as the inputs to the PFD. 


\subsection{Serial Interface}

Some of the circuit blocks have static control signals, as listed in Table.4.4.

\begin{tabular}{|l|l|l|l|}
\hline Signal Name & Description & Width & Default Value \\
\hline int $N<6: 0>$ & Base division ratio for the N-divider & 7 & $7 b^{\prime} 1100010$ \\
\hline inoise $<2: 0>$ & Noise control on Charge Pump & 3 & $3 b^{\prime} 111$ \\
\hline ipump $<1: 0>$ & Current control on Charge Pump & 2 & $2 b^{\prime} 11$ \\
\hline multi_bit & Switch between single-bit mode and multi-bit mode & 1 & 1 'b1 \\
\hline dwa_enable & Enable or disable DWA capability & 1 & 1 'b1 \\
\hline
\end{tabular}

Table 4.4: Static Control Signals

Because there is a limited number of pads, it is impossible to assign a pad for each of the control signals. A Serial Interface (SIF) is used to overcome this problem. A simple and straight-forward method to implement serial-to-parallel conversion is shown in Fig.4.34.

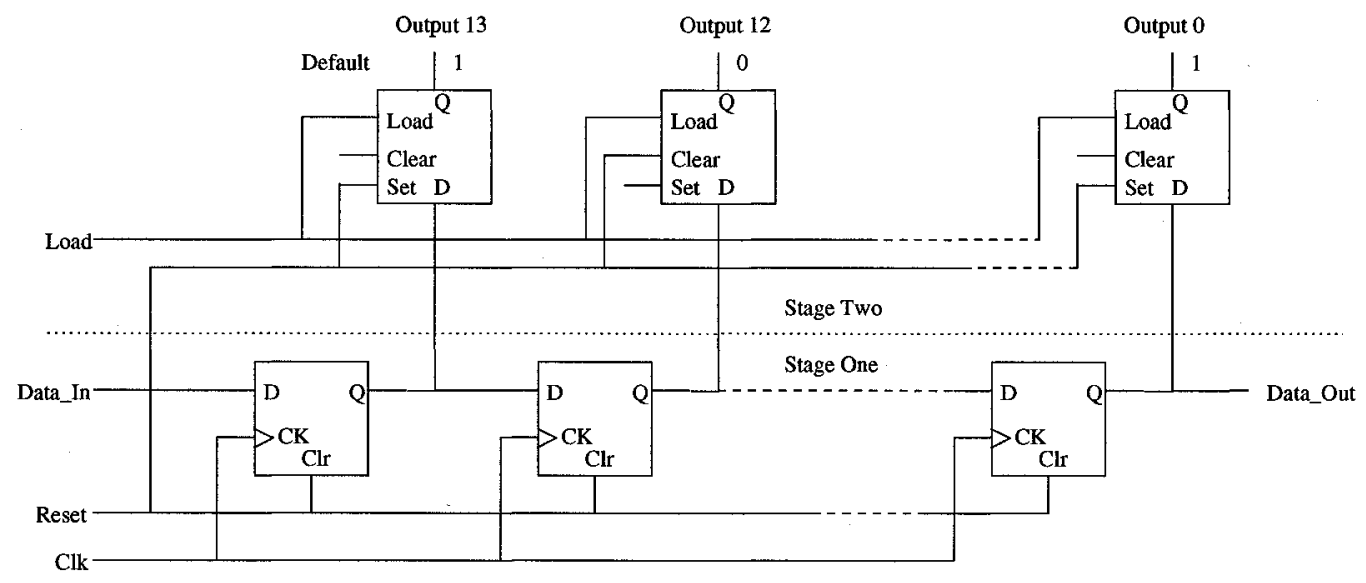

Figure 4.34: Implementation of Serial Interface 
A two-stage approach is taken. In stage one, the data is shifted into a register chain at the rising edge of the clock. After fourteen of these clock cycles, the old set of control signals will have been replaced by a new set. In stage two, the output parallel register is triggered and the new set becomes available.

When the Reset signal is active, all outputs are reset to their default values. (Default values which are determined by wiring the Reset signal to the appropriate Clear and Set inputs of the register).

Table.4.5 describes each of the block terminals:

\begin{tabular}{|l|l|l|}
\hline Name & Description & I/O \\
\hline Reset & when high, initializes interface & Input \\
\hline Clock & common FF clock & Input \\
\hline Load & strobes shift register contents to output register & Input \\
\hline DataIn & Serial data input & Input \\
\hline DataOut & Serial data output (for testing) & Output \\
\hline
\end{tabular}

Table 4.5: Serial Interface Signals

The number of signal inputs needed for the items of Table.4.4 is four. 
The timing diagram for the SIF is shown in Fig.4.35.

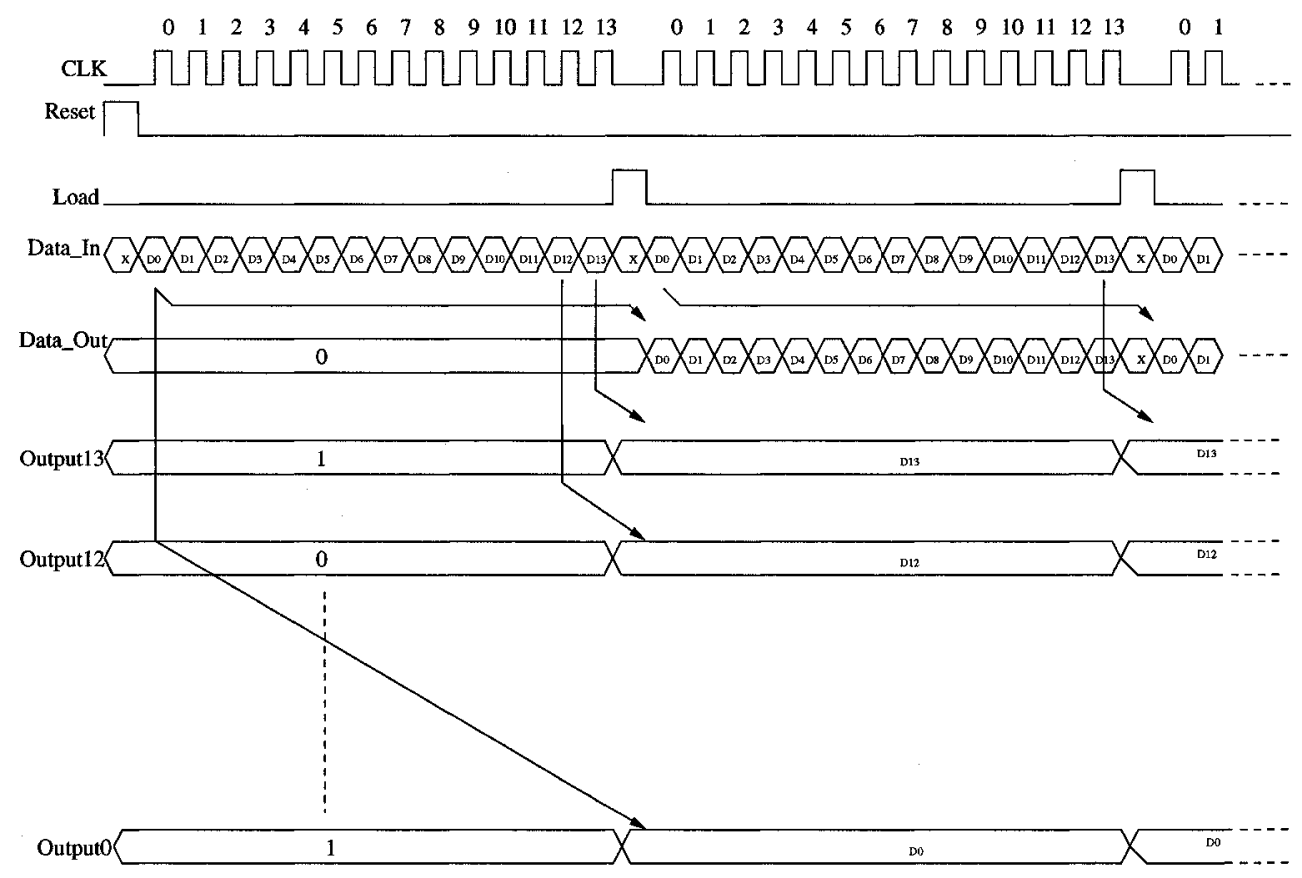

Figure 4.35: Serial Interface Timing 
An RTL simulation of the SIF was performed, with results shown in Fig.4.36.

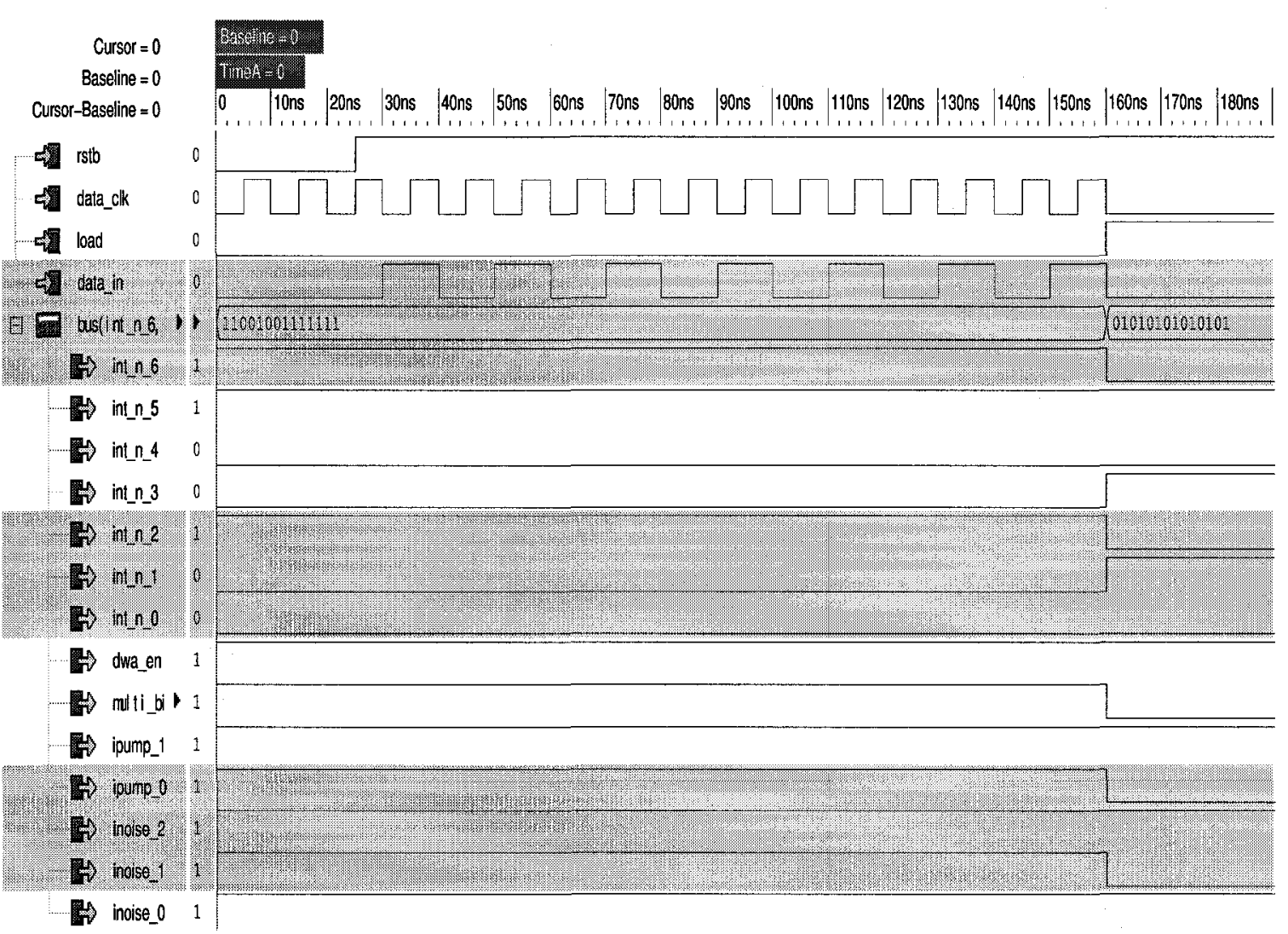

Figure 4.36: Serial Interface RTL Simulation

The SIF outputs are set to their initial values when the Reset is active, further SIF operation is inhibited until Reset is inactive. Data is then shifted in with each rising edge of the clock and after fourteen clock cycles, the data pattern 01010101010101, for example, is present at the shift-register outputs. The Load clock can then be used to transfer the data pattern to the output registers. 


\subsection{Overall Performance}

The entire system was simulated using SpectreVerilog with each circuit block modelled with Verilog-A, Verilog, or at the transistor level. The results are summarized in Table.4.6.

\begin{tabular}{|c|c|c|c|c|c|c|}
\hline Fig\# & MMD & PFD & CP & Quantizer & Integrator & DFB \\
\hline Fig.4.37 & Verilog & Verilog & Verilog-A & Verilog-A & Verilog-A & Verilog \\
\hline Fig.4.38 & Verilog & Transistor Level & Verilog-A & Verilog-A & Verilog-A & Verilog \\
\hline Fig.4.39 & Verilog & Verilog & Transistor Level & Verilog-A & Verilog-A & Verilog \\
\hline Fig.4.40 & Verilog & Verilog & Verilog-A & Transistor Level & Verilog-A & Verilog \\
\hline
\end{tabular}

Table 4.6: System SpectreVerilog Simulations

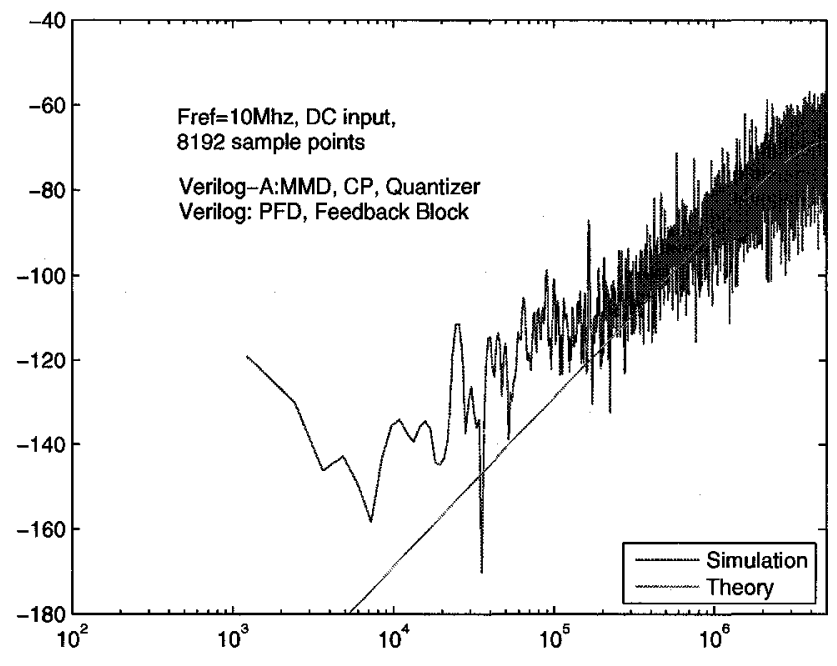

Figure 4.37: System Simulation with Verilog-A and Verilog 


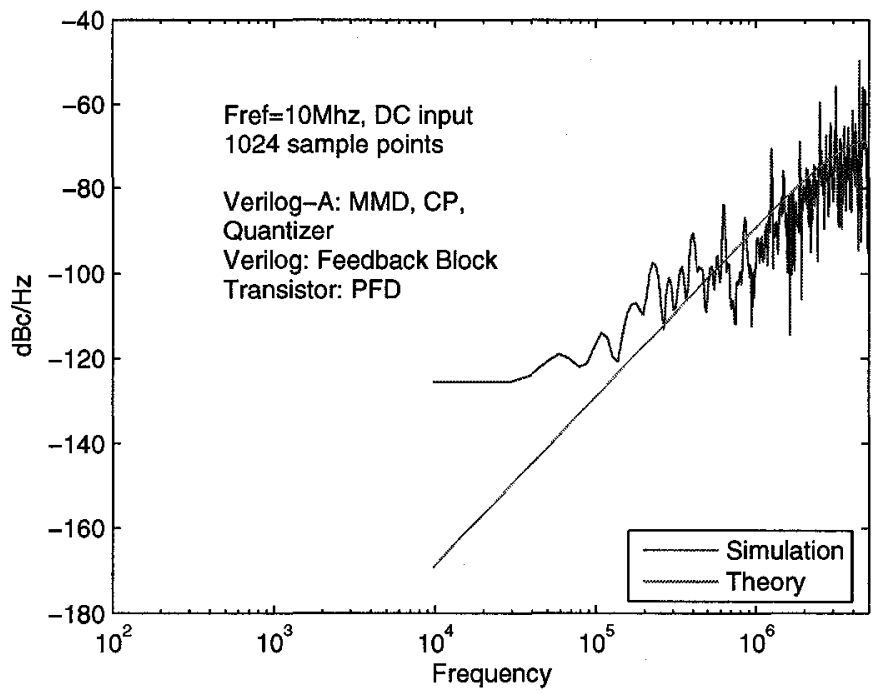

Figure 4.38: System Simulation (PFD Modelled at the Transistor Level)

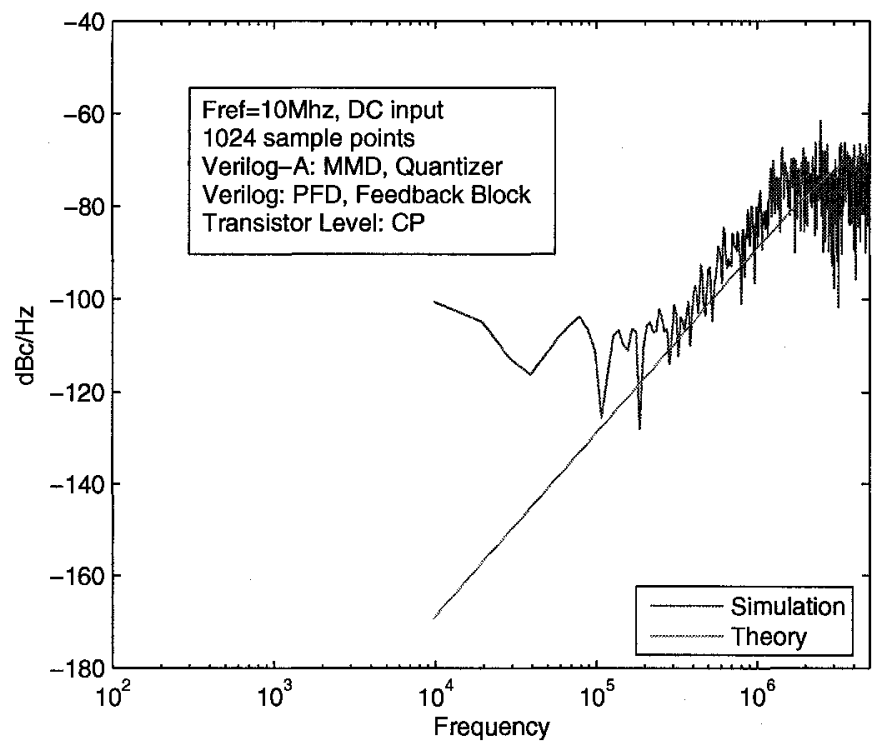

Figure 4.39: System Simulation (CP Modelled at the Transistor Level) 


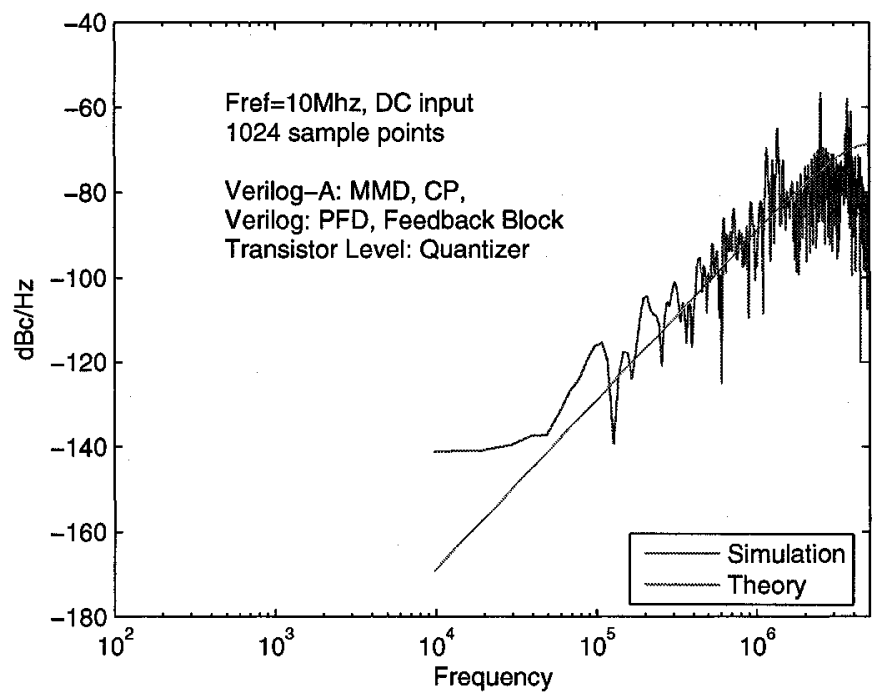

Figure 4.40: System Simulation (Quantizer Modelled at the Transistor Level)

The noise data collected from transistor level simulations of each of the blocks was applied to the appropriate transfer function as described in Section 3.7. The frequency noise performance is shown in Fig.4.41.

Simulations with the PFD or CP modelled at the transistor level show a significant increase in the noise floor at lower frequencies (Fig.4.38 and Fig.4.39). This is most likely caused by non-linearity in the PFD and CP folding high frequency noise back to lower frequencies [RFDK03]. The input referred frequency noise performance is shown in Fig.4.41. This is obtained by multiplying the output binary data by $\frac{f_{\text {ref }}}{8}$ ).

The in-band (10 kHz to $200 \mathrm{kHz}$ ) SNR figure calculated with the addition of the simulated noise is $42.46 \mathrm{~dB}$, down from $46.68 \mathrm{~dB}$ when only quantization noise was considered.

The phase noise can be obtained using:

$$
S_{\delta \phi}(f)=\frac{1}{f^{2}} S_{\delta f}(f)
$$




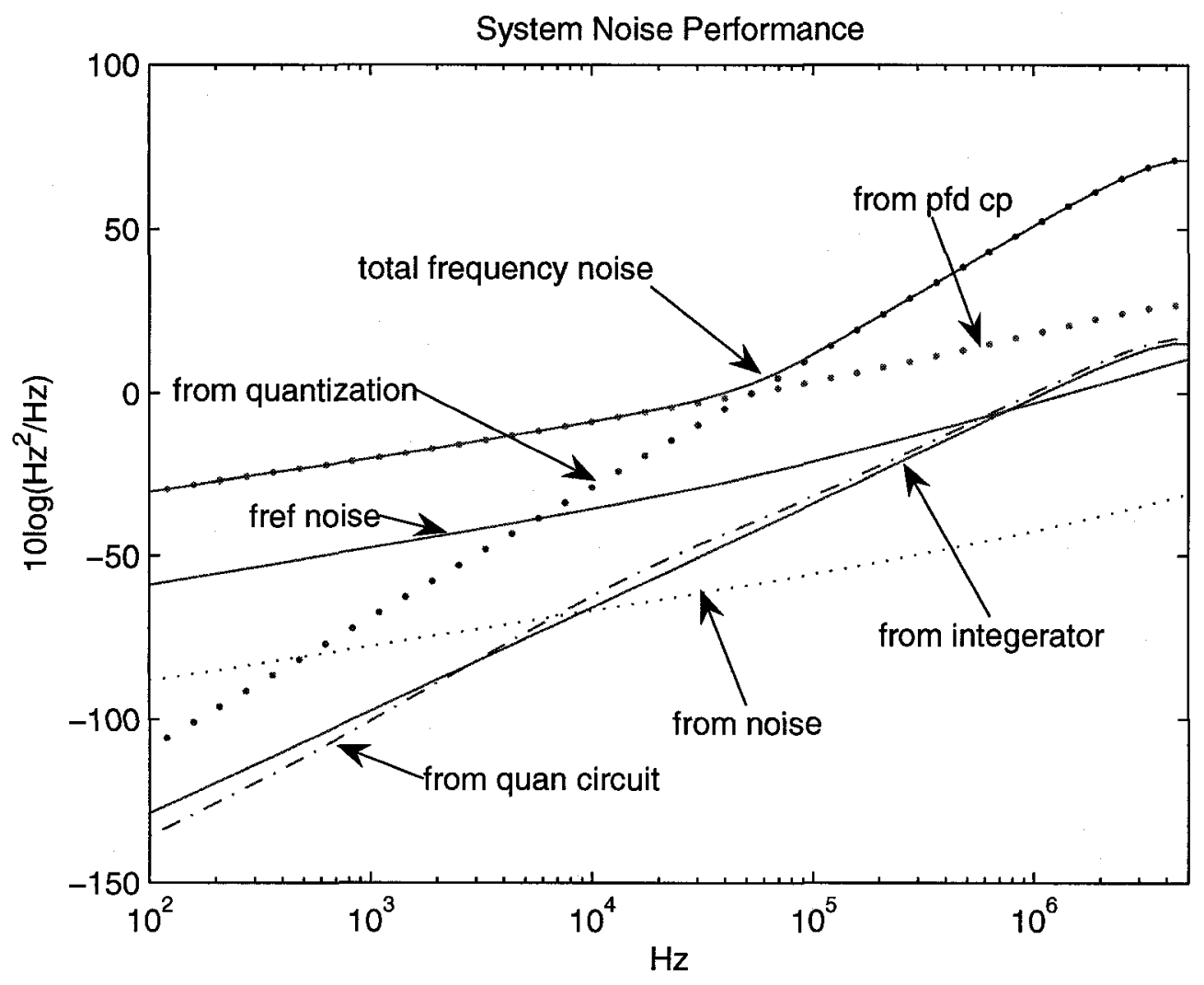

Figure 4.41: System Frequency Noise Performance

Where $S_{\delta \phi}(f)$ is the spectral density of the phase noise and $S_{\delta f}(f)$ is the spectral density of the frequency noise shown in Fig.4.41.

The phase noise is plotted in Fig.4.42. These results indicate that if the discriminator were used as part of frequency synthesizer with a closed loop bandwidth of $100 \mathrm{kHz}$, the in band phase noise, due to the discriminator, would be $-90 \mathrm{dBc} / \mathrm{Hz} @ 20 \mathrm{kHz}$ offset for an output frequency of $1 \mathrm{GHz}$ and a reference frequency of $10 \mathrm{MHz}$. This indicates a BFM [RFDK03] of $-90 \mathrm{dBc} / \mathrm{Hz}-20 \log 10(\mathrm{~N}=100)-10 \log 10(\mathrm{Fref}=10 \mathrm{MHz})=-200$. 


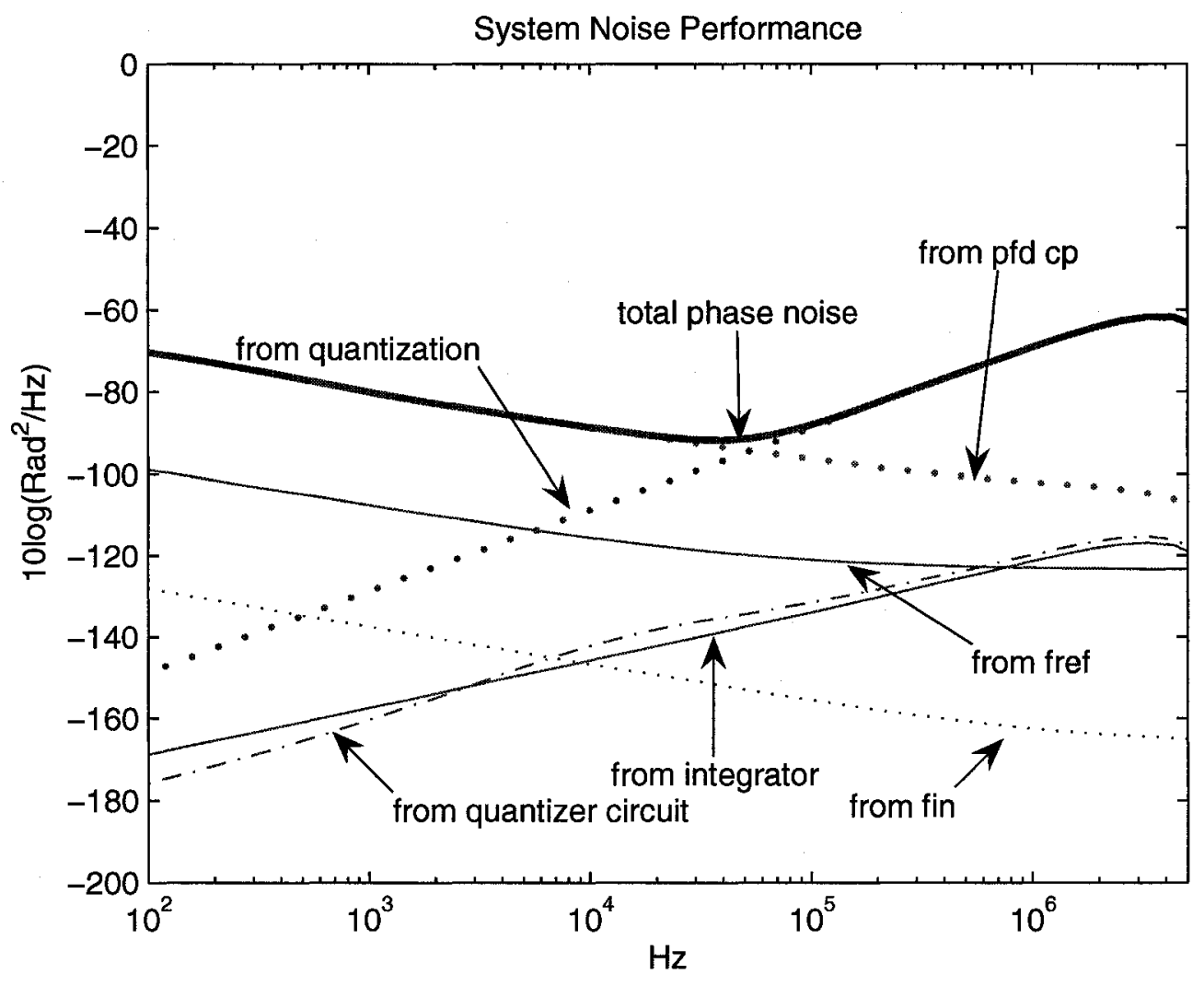

Figure 4.42: System Phase Noises Performance

At low frequencies, the noise from the $\mathrm{CP}$ and the PFD is dominant. Improvement of the $\mathrm{CP}$ and PFD noise performance is listed as an item for future work. 


\section{Chapter 5}

\section{Measurements}

\subsection{Chip Layout and Test Setup}

The chip is housed in an 80-pin Ceramic Flat Package (CFP), with the test fixture being provided by the Canadian Microelectronics Company:(http://www.cmc.ca).

A plot of the chip layout is shown in Figure. 5.1. 


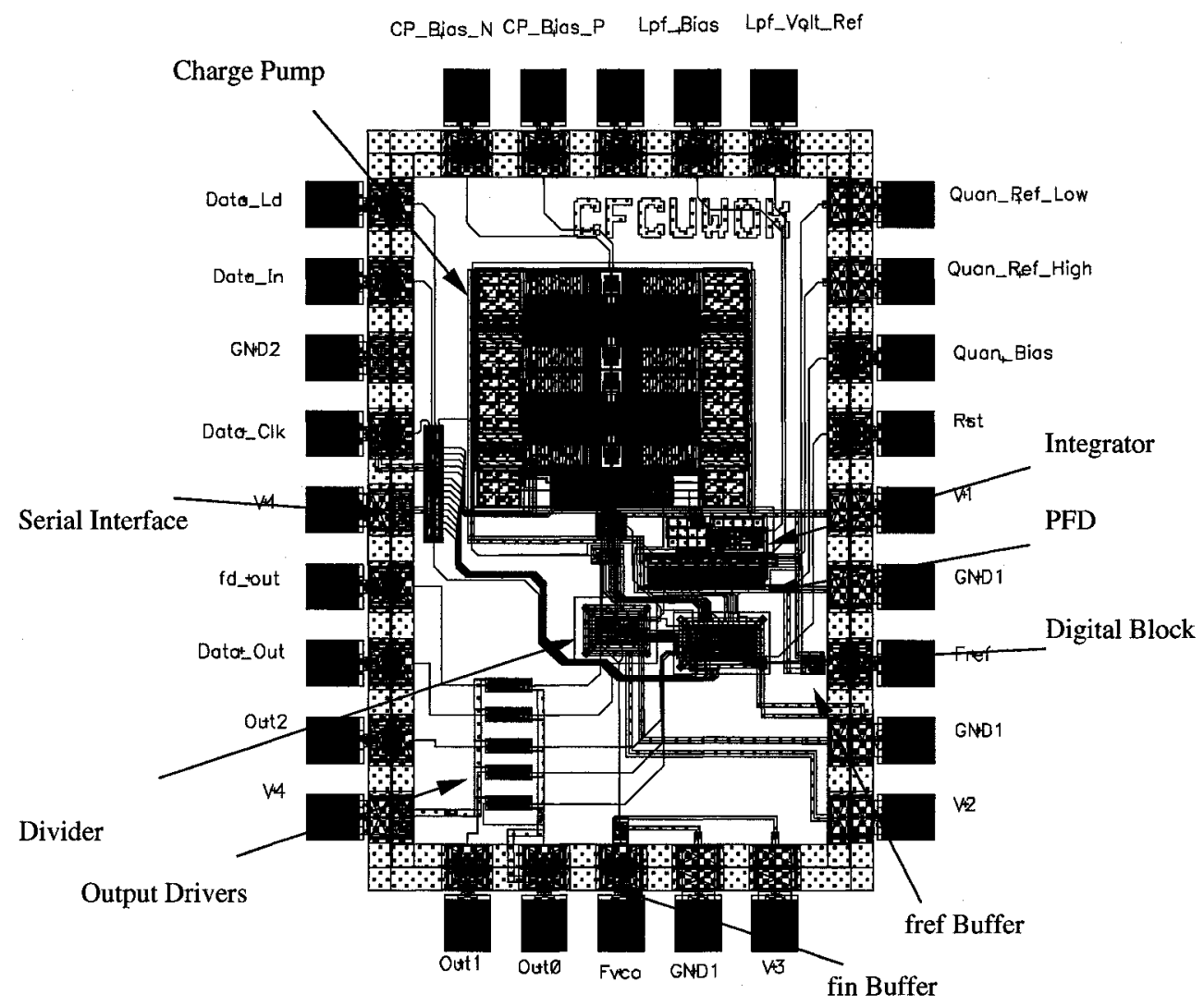

Figure 5.1: Chip Layout 
The proposed test setup is shown in Figure 5.2. An RF signal generator is used to provide both the $f_{\text {in }}$ input frequency and the $10 \mathrm{MHz}$ reference clock (the latter from the generator's clock reference output). A digital pattern generator provides a serial data stream to the Serial Interface, and the 3-bit output stream is sent to a logic analyzer. An oscilloscope is connected to the output of the Multi-Modulus Divider for debugging purposes.

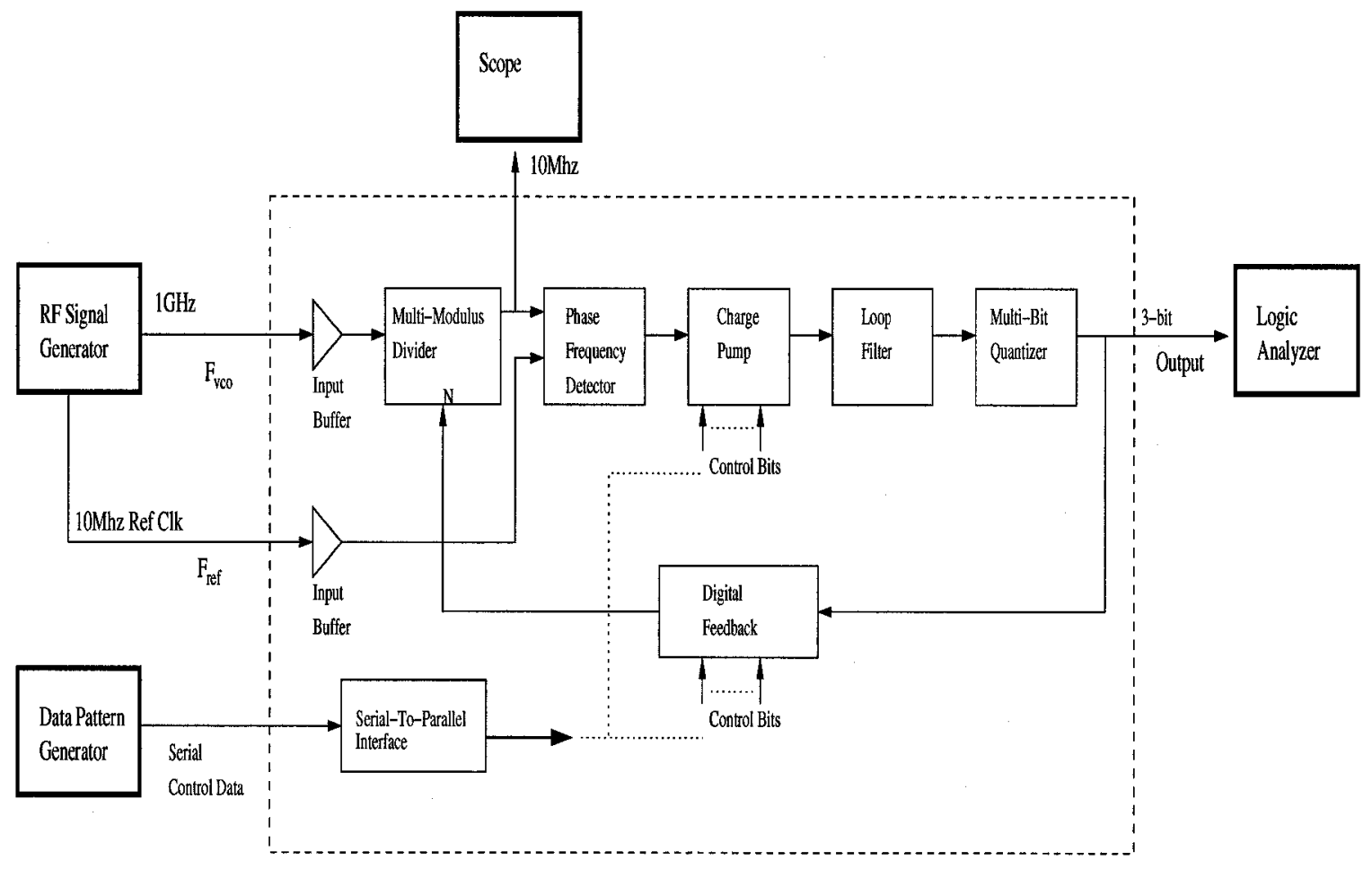

Figure 5.2: Test Setup 


\subsection{Fabrication and Fault Analysis}

Due to various implementation problems, three designs were fabricated and tested. The details for each fabrication run are given in the following paragraphs.

\section{First fabrication:}

A design error was found after the layout was submitted: the CP input polarity was reversed, resulting in positive, rather than negative feedback, in the system loop. This prevented the loop from locking and it was decided to submit a second design for fabrication.

\section{Second fabrication:}

For the second submission, transistor level simulations showed correct operation. The measurement results on DC bias levels of the external pins were very close to those obtained from simulations, as shown in Table.5.1.

\begin{tabular}{|c|c|c|c|}
\hline Signal Name & Description & Simulation Result & Measurement Result \\
\hline$f_{\text {in }}$ & Input Clock & $0.78 \mathrm{v}$ & $0.75 \mathrm{v}$ \\
\hline$f_{\text {ref }}$ & Reference Clock & $0.80 \mathrm{v}$ & $0.76 \mathrm{v}$ \\
\hline bias $n$ & CP NMOS bias & $0.45 \mathrm{v}$ & $0.43 \mathrm{v}$ \\
\hline bias_p & CP PMOS bias & $1.27 \mathrm{v}$ & $1.30 \mathrm{v}$ \\
\hline$l f_{\text {ref }}$ & Integrator Ref & $0.53 \mathrm{v}$ & $0.51 \mathrm{v}$ \\
\hline
\end{tabular}

Table 5.1: External Pin DC Bias Level Measurement

However, $f_{\text {div }}$, the $10 \mathrm{MHz}$ output signal, from the Multi-Modulus Divider was not detected during testing. The vendor-supplied 'Standcell' output drivers were thought to be at fault, but verification of the suspicion was necessary. In particular, it had to be shown that the $10 \mathrm{MHz} f_{d i v}$ signal is present at the output of the Multi-Modulus Divider.

For monetary reasons, it was decided to forgo a 'Micro-Surgery' procedure in favor of 
the noninvasive Dynamic Electroluminescence Imaging (DEI) technique [HFSN04]. This method is based upon the fact that passage of current through any semiconductor (it doesn't have to be a material expressly designed to emit photons, as in LEDs) is accompanied by the emission of detectable amounts of light. By observing an operating chip with a microscope and a high-gain photo-detector it is possible to display, both spatially and temporally, the current flow at the circuit nodes of interest.

A block diagram for a DEI system is shown in Fig.5.3

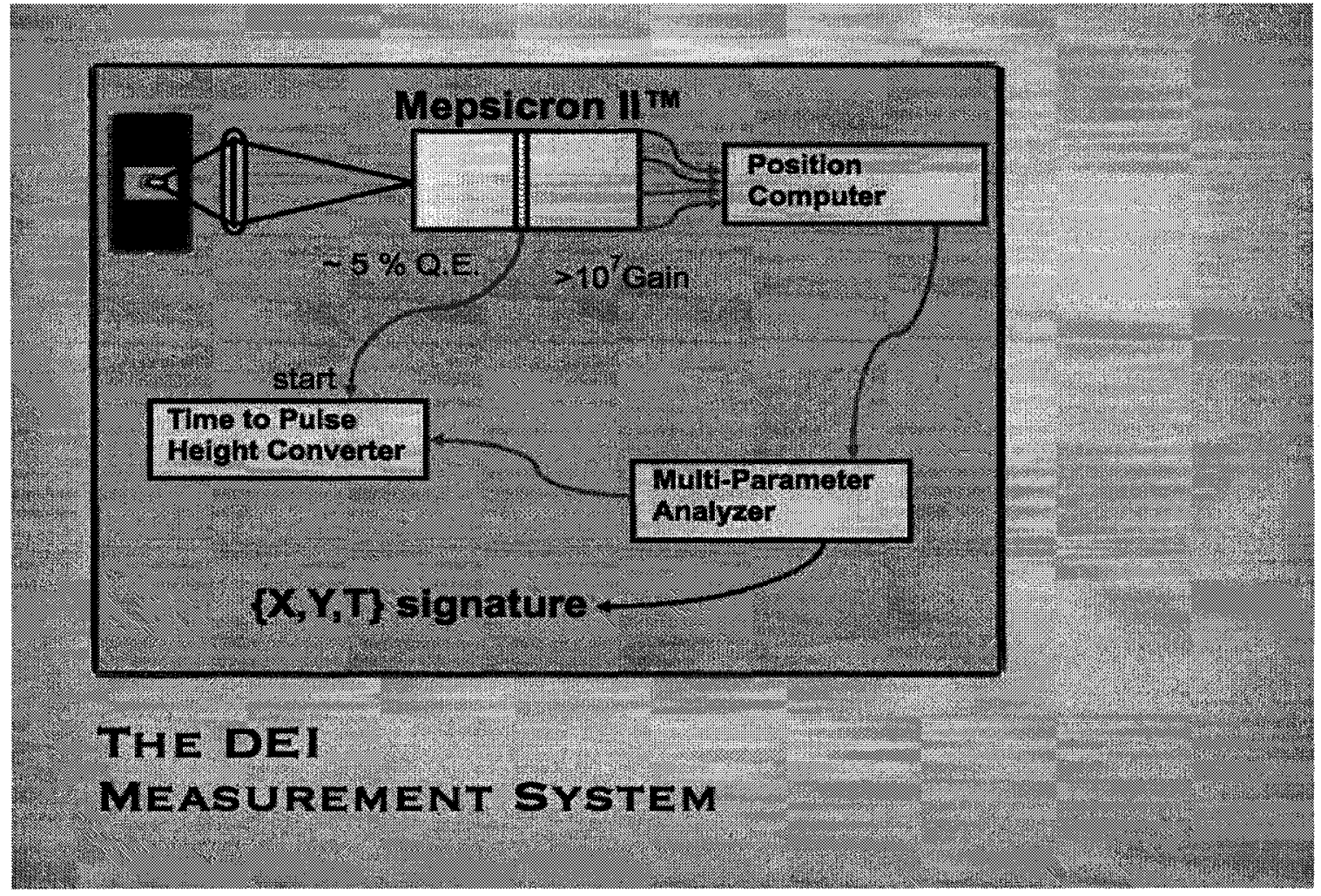

Figure 5.3: Block Diagram for a DEI System [HFSN04]

The light collection is performed with a conventional microscope and imaged onto the photo-cathode of a position dissecting photomultiplier tube called a Mepsicron $I I^{T M}$ [HFSN04]. 
The tube is configured for single photon counting so that each detected photon can be processed separately. The Mepsicron provides five output signals, four for a position computer and one to start the Time to Pulse Height Converter (TPHC). The outputs of the Position Computer and the TPHC are captured by a multi-parameter analyzer and stored as a stream of events with $X, Y, T$ signatures.

The test setup using the DEI system is shown in Fig.5.4

\section{DEI System}

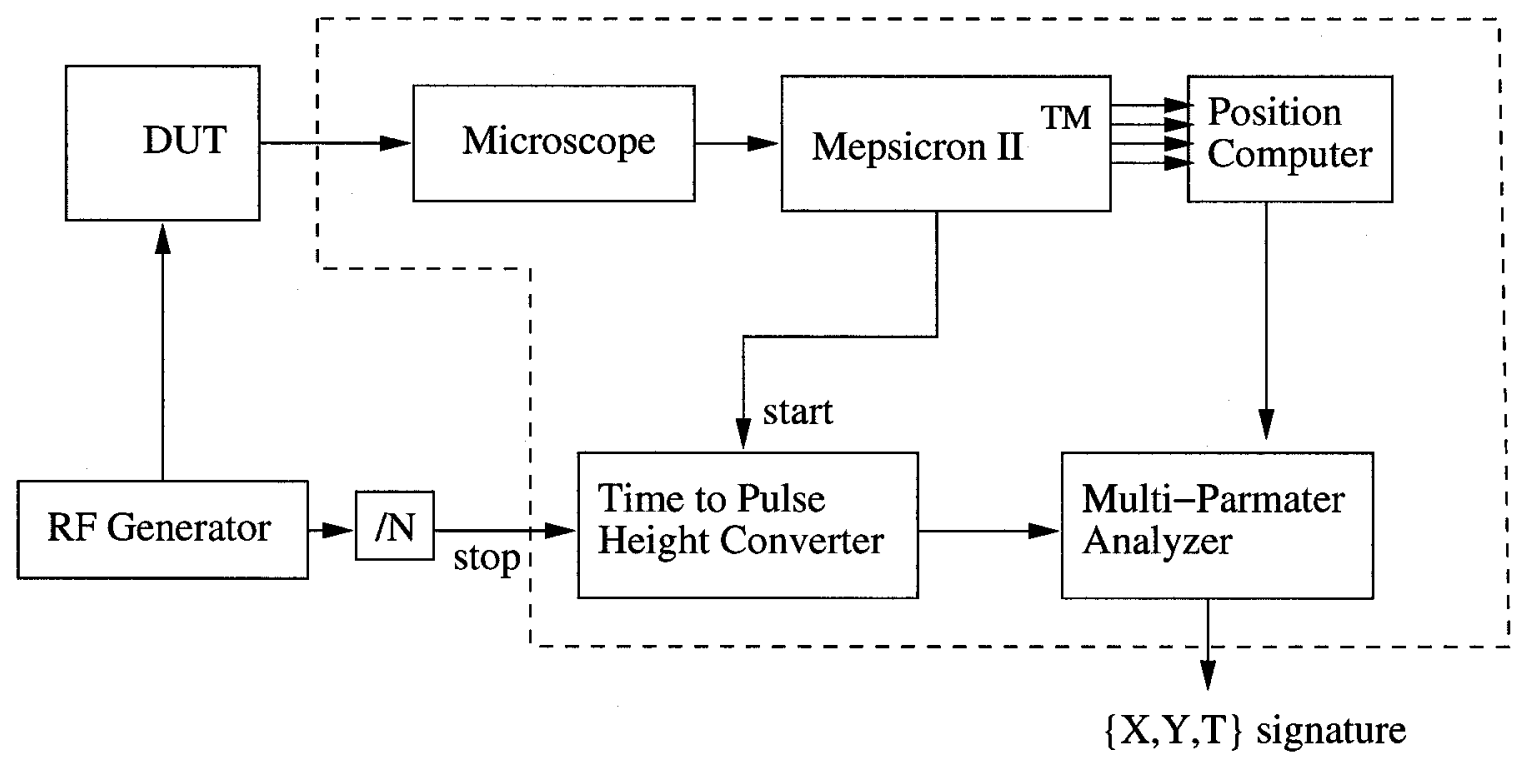

Figure 5.4: Test setup with DEI System

The RF signal generator provides the $1 \mathrm{GHz}$ input signal, $f_{\text {in }}$, to the Device Under Test (DUT). The $1 \mathrm{GHz}$ signal is also divided down to $2.5 \mathrm{MHz}$ to give a STOP signal for the TPHC. If the DUT is working properly, four cycles of the waveform for the expected 10 $\mathrm{MHz} f_{\text {div }}$ output can be captured in the $40 \mathrm{~ns}$ long window.

The area of the Multi-Modulus Divider on the die was searched for signs of the $10 \mathrm{MHz}$ $f_{d i v}$ signal. In that area, the $10 \mathrm{MHz} f_{d i v}$ signal is mixed with signals of various frequencies 
$\left(f_{\text {in }} / 3, f_{\text {in }} / 4, \ldots, f_{\text {in }} / N\right)$. The DEI technique has the ability of detecting the $10 \mathrm{MHz} f_{d i v}$ signal among the others, as will be shown later. The location of the Multi-Modulus Divider is illustrated in Fig.5.5.

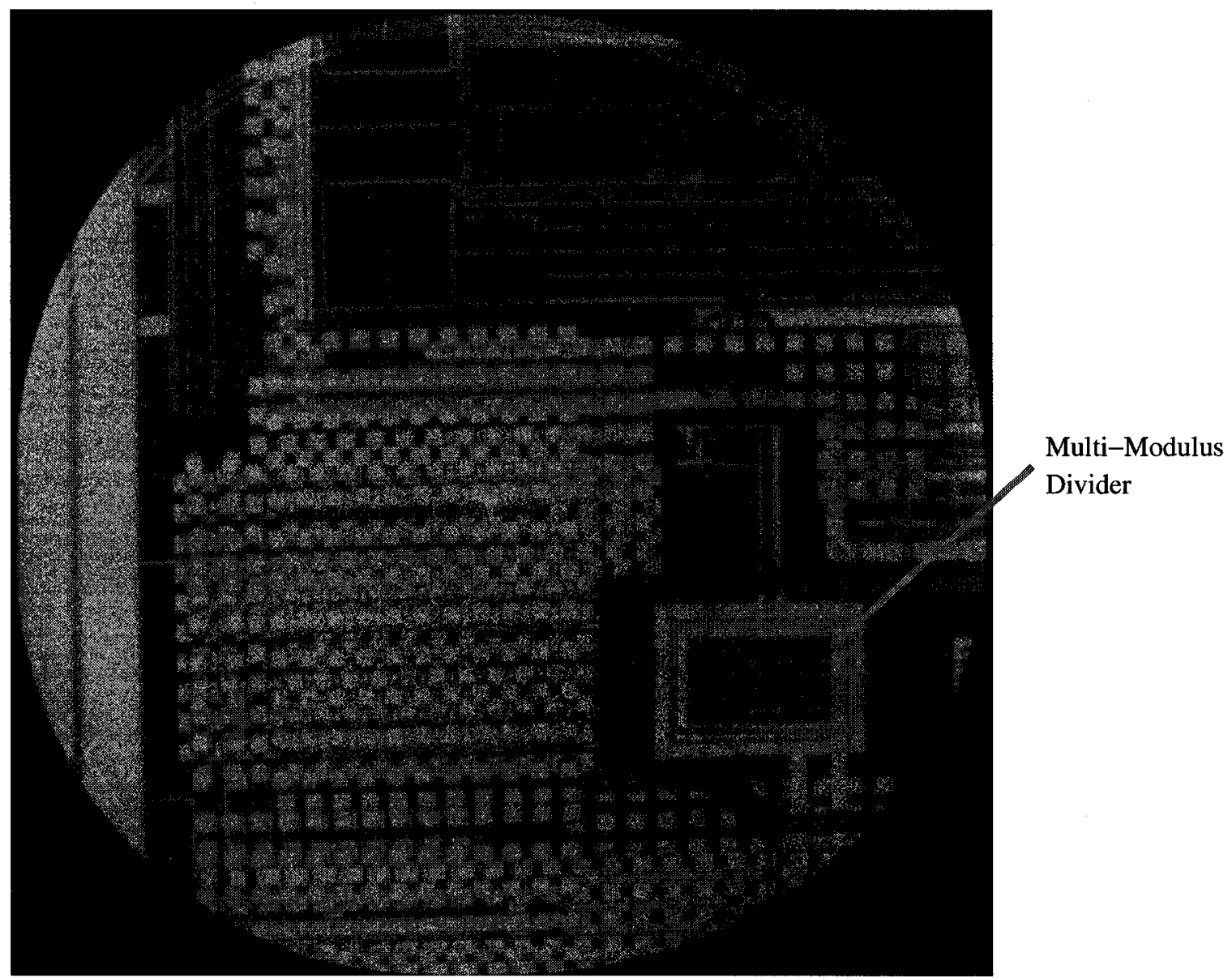

Figure 5.5: Locations for DEI Measurements 
When Reset is inactive, the Multi-Modulus Divider is operating, with divider transistors switching at various fractions of $f_{\text {in }} \mathrm{Hz}$, that is, at $f_{\text {in }} / 3, f_{\text {in }} / 4, \ldots, f_{\text {in }} / N$. The electroluminescence of the transistors will form a 'cloud' in the DEI image as shown at the bottom of the left part of Figure 5.6. On the other hand, when Reset is active, the Multi-Modulus Divider only has the $f_{\text {in }}$ input present, and a bright spot is shown in the captured image (bottom of the right part of Figure 5.6).
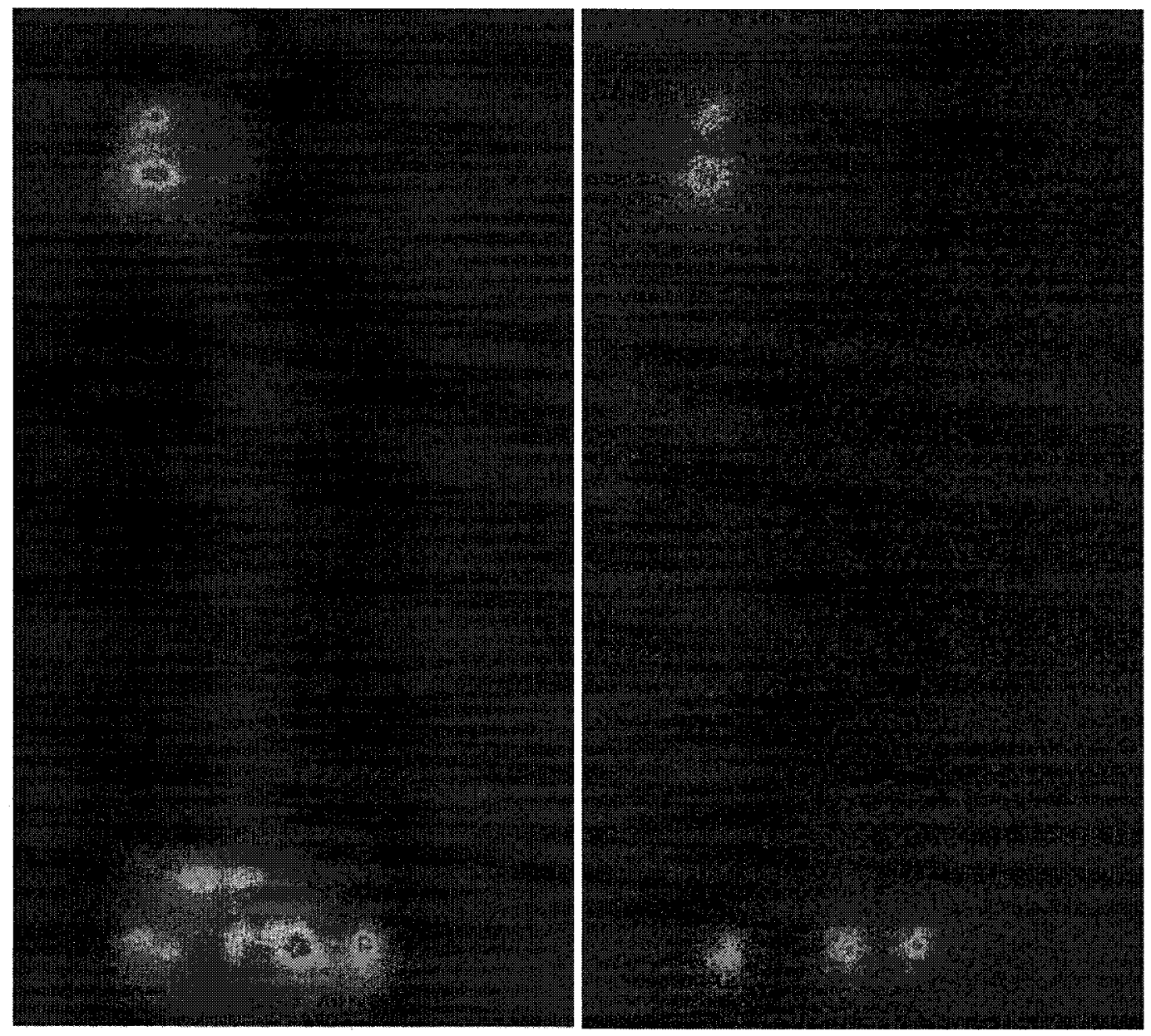

Figure 5.6: DEI Images. Left: RESET is inactive and Right: RESET is active 
The above images were processed to produce the time-correlated waveforms, shown in Fig.5.7 and Fig.5.8.

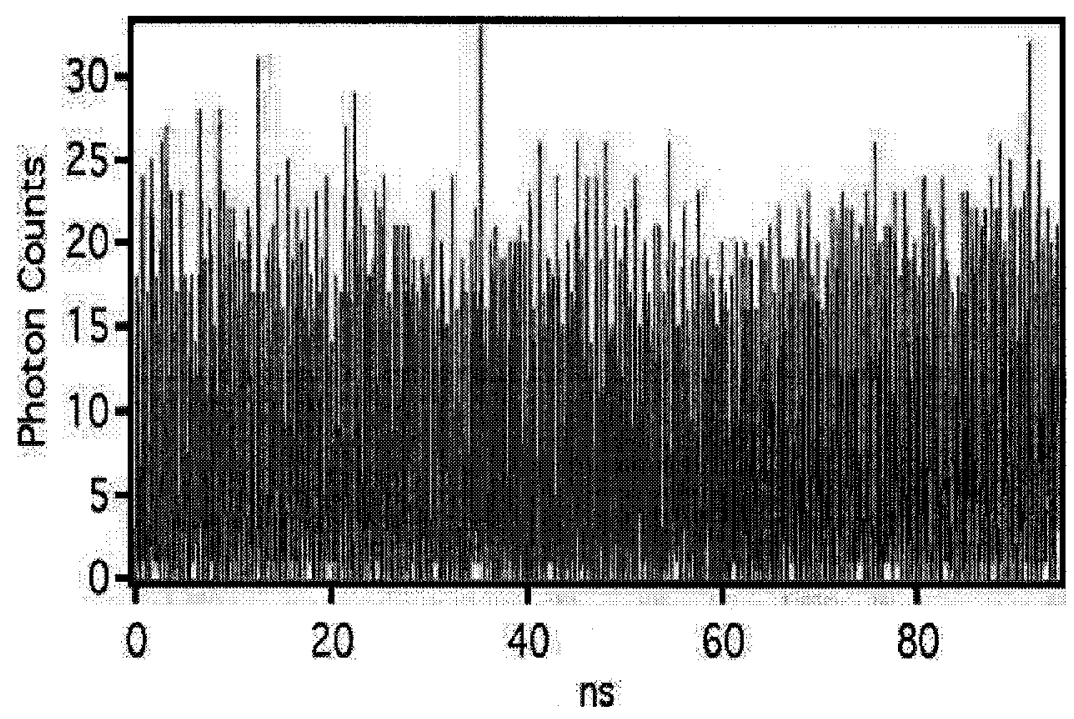

Figure 5.7: Time-Correlated Waveform When RESET is active

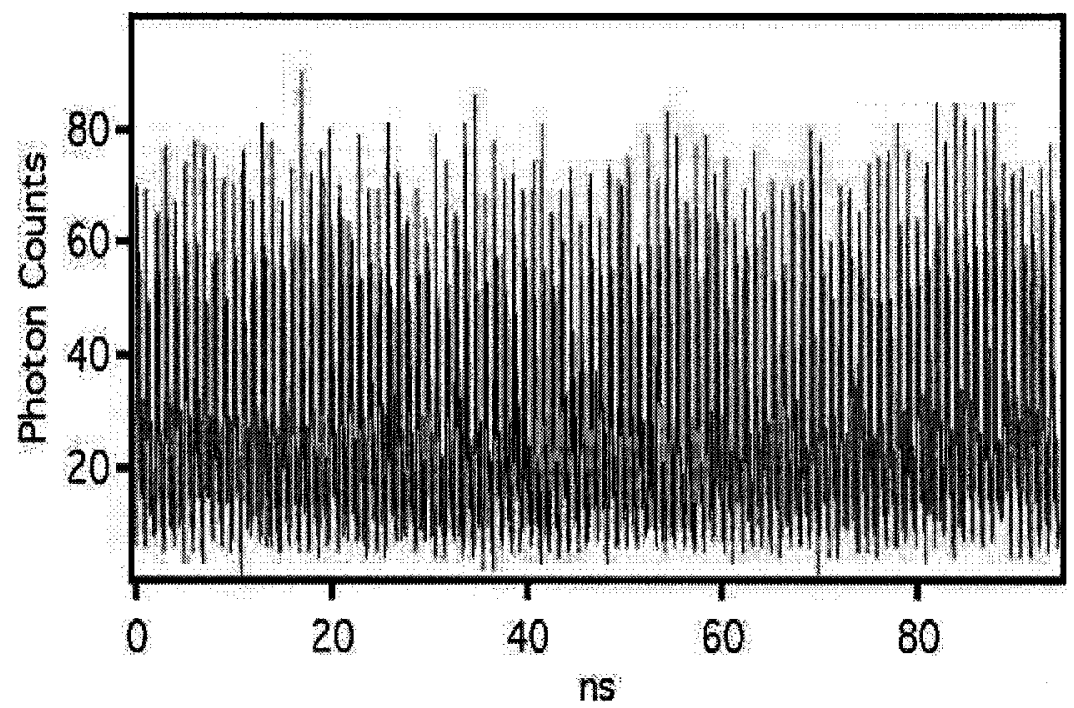

Figure 5.8: Time-Correlated Waveform When RESET is inactive 
The only visible difference between Fig.5.7 and Fig.5.8 is the amount of the electroluminescence (photo counts). In both cases, only $1 \mathrm{GHz}$ and its harmonics were seen, although the intensity patterns were different. With RESET active, the overall intensity was weaker. Although the difference between when RESET was active and inactive suggested the Multi-Modulus Divider was operating, no $10 \mathrm{MHz}$ signal could be identified from the signals shown in Fig.5.8. ( Later with the third fabrication, and extra information obtained from electrical testing, it was determined the reason no $10 \mathrm{MHz}$ was detected is that the wrong trigger frequency was used: The Multi-Modulus Divider was dividing by 101 , not 100 , which makes $f_{d i v}=9.9 \mathrm{MHz}$, not synchronous with the trigger frequency 2.5 MHz.)

As the $10 \mathrm{MHz} f_{d i v}$ signal couldn't be identified, the DEI measurement results were not conclusive for the second fabrication. 


\section{Third fabrication:}

For the third fabrication, the presumably defective 'Standcell' output drivers were replaced with custom-designed drivers consisting of cascaded CMOS inverters [WE02]. The $10 \mathrm{MHz} f_{d i v}$ output signal from the Multi-Modulus Divider was detected using an electrical oscilloscope.

Figure 5.9 shows that the duration of the high state of the divider output is four $f_{\text {in }}$ cycles as expected.

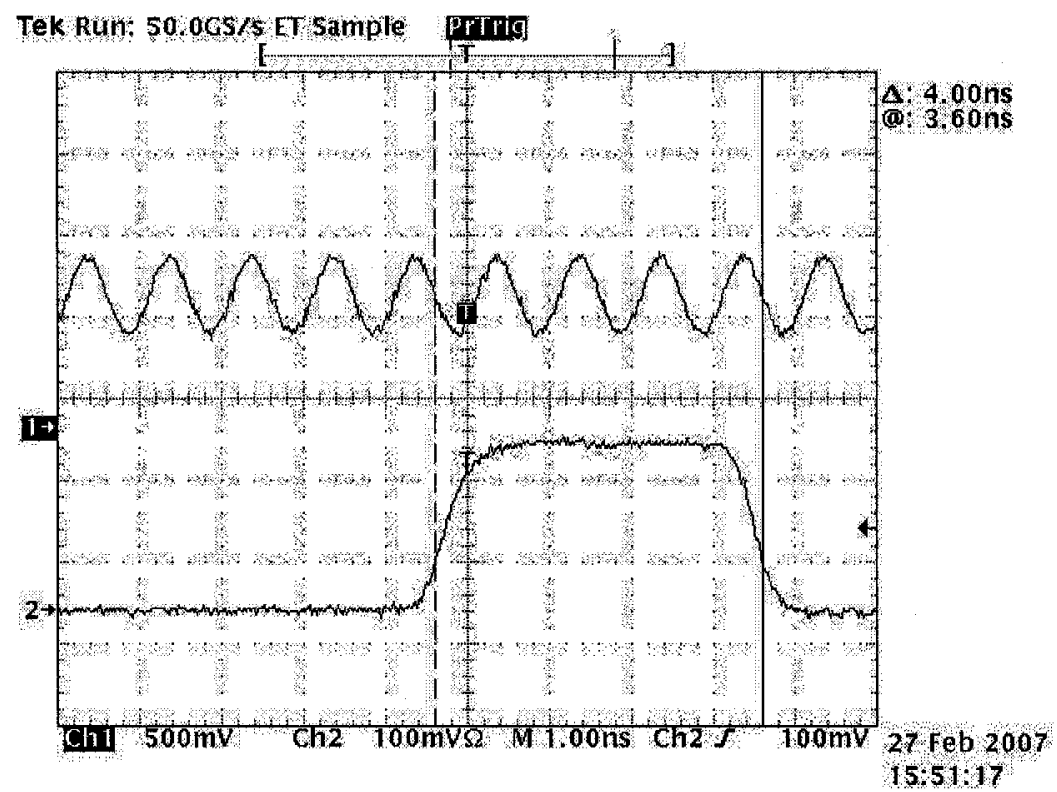

Figure 5.9: Divider Waveform

Unfortunately, two bonding wires were mistakenly interchanged by the bonding facility and full testing will be impossible until this defect is repaired.

DEI measurements were performed again on the third fabrication. With the extra information obtained from the electrical testing, the right trigger frequency was used. The waveform from the Multi-Modulus Divider area when RESET is inactive is shown in Fig.5.10. 


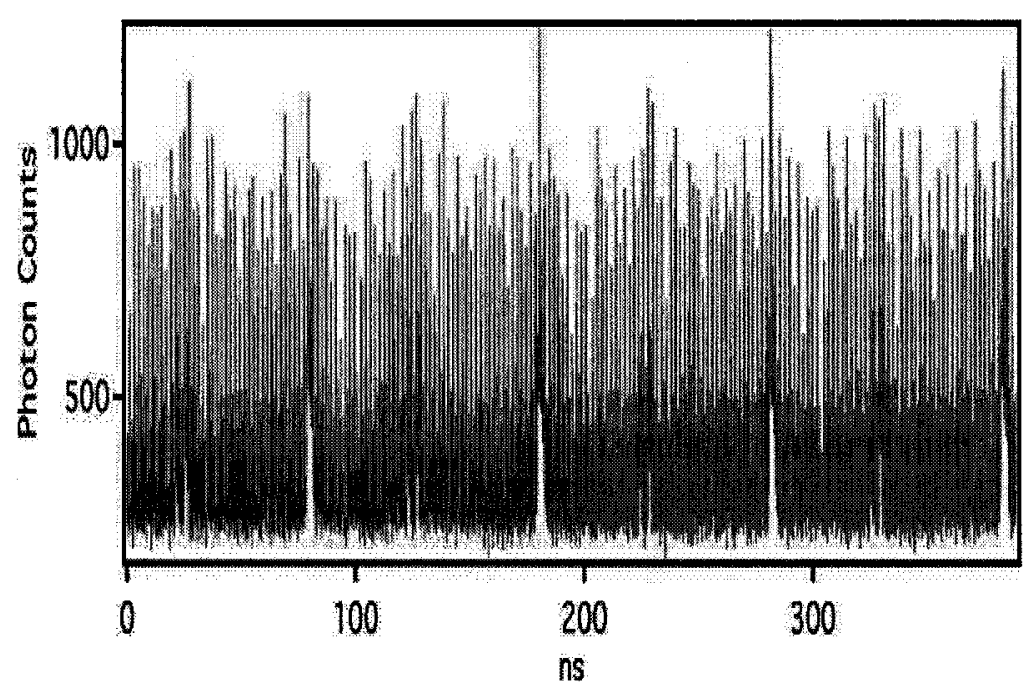

Figure 5.10: Time-Correlated Waveform When RESET=OFF

It is shown that the $10 \mathrm{MHz}$ output signal exists among the other signals. Had the correct division ratio been used in earlier testing, the same waveform would have been measured for the second fabrication.

More DEI measurements are planned for the second and the third fabrication. Signals will be traced along different paths using the DEI technique. Those measurements will show the internal circuits are operating without unduly loading the circuits.

Full electrical testing of the system will be done when the bonding wires are repaired. However, such testing is beyond the scope of this thesis. The results from previous measurements and the co-operation test work with the NRC and MuAnalysis Inc. have resulted in a submission to CSTC 2007[HSF ${ }^{+}$07]. 


\section{Chapter 6}

\section{Conclusions and Future Work}

\subsection{Conclusions}

\subsubsection{Summary}

The motivation for this thesis was to look at a new architecture for a $\Delta \Sigma$ FD that would be suitable for second-generation frequency synthesis applications. Such synthesizers allow the loop filter to be implemented on chip in the digital domain while maintaining low phase noise. Digital implementation allows the loop filter to be programmed on the fly enabling multi-mode wireless.

A brief review of different types of frequency discriminators was presented, and then the thesis focused on the development of a $\Delta \Sigma$ FD. Different existing architectures for $\Delta \Sigma$ FD were examined closely. Performance of each of the $\Delta \Sigma$ FDs was compared. The results were provided as the benchmark for a new design for a $\Delta \Sigma$ FD that is the first known to employ a fractional-N divider.

This new design uses a fractional-N divider in order to reduce quantization noise and 
to achieve finer frequency resolution and higher in-band SNR. In a $\Delta \Sigma$ FD that uses an integer- $\mathrm{N}$ divider, the quantization step size, $\Delta$, is $F_{\text {ref }}$. By using a $1 / 8$ fractional-N divider, the quantization step is reduced to $F_{r e f} / 8$. As quantization noise is directly proportional to the quantization step, reducing $\Delta$ by eight results in an $18 \mathrm{~dB}$ total quantization noise power reduction. In this new design, the fractional-N divider is implemented in a novel way: The multi-modulus divider has two outputs: $f_{d_{0}}$ and $f_{d_{1}} \cdot f_{d_{0}}$ is one input frequency cycle ahead of $f_{d_{1}}$. These two signals, along with the PFD and CP array, form a D/A for cancelling out the truncation error remaining in the Accumulator. Other existing architectures for fractional-N dividers incorporate a dedicated high precision D/A at the output of the accumulator in the feedback path and focus on techniques to match the D/A charge transfer with the charge transferred through the PFD and CP (see Fig. 3.10). In this novel architecture, most of the components making up the D/A are in the PFD and CP array so that charge transfer mismatch from these components is eliminated. The mismatches that cannot be eliminated (except through careful layout techniques) result from $f_{d_{1}}$ not being exactly one cycle delay or from delay mismatches in the different paths. This approach take advantage of the ability to apply Dynamic Element Matching techniques to the mismatches between the eight parallel paths (each path consists of a Selector element, PFD and $\mathrm{CP}$ ). The elimination of the precision $\mathrm{D} / \mathrm{A}$ circuit makes the system more suitable for implementation in a CMOS process. A 3-bit quantizer is used to restore the input range.

Matlab simulations of this new $\Delta \Sigma$ FD were conducted to predict performance and validate the architecture. A linear model of the system was created. The transfer functions from each block in the system to the system output were derived from the linear model. The impact on performance from different choices of system parameters was evaluated both analytically and experimentally (by simulation). Several system level techniques were introduced to mitigate circuit level non-idealities. The analysis and simulations prove the 
concept of the new $\Delta \Sigma$ FD works. The results from the system analysis and simulation were captured in a conference paper [FFRC07] that was accepted by ISCAS (International Symposium on Circuits and Systems) 2007.

Circuits for each block in the system were designed in TSMC $0.18 \mu \mathrm{m}$ CMOS process. The system was simulated using SpectreVerilog with different blocks simulated at the Verilog, Verilog-A or transistor level as appropriate. In each block, transistor level simulations were conducted to verify the functionality of the block and to collect noise data. The noise data was plugged into the transfer functions developed in the linear analysis in order to predict the total noise output of the system. The design was fabricated three times. The first one was not successful due to a design error. The second one was difficult to test due to a non-functional "Standcell" output driver. The third chip is currently under testing. Due to a bonding error at the packaging facility, only a small part of the system has been tested and shown to be working.

DEI analysis techniques were used to do fault analysis on the second fabrication. Microsurgery and micro-probing were considered initially, but the protracted and costly test cycles of those techniques made them less attractive. DEI is a low-cost, noninvasive, nondestructive optical approach and it has bandwidth approaching $100 \mathrm{GHz}$. Even with the circuits under six-layer metal masks, DEI was able to successfully detect the light emitted by the transistor gates when switching. Light was collected with a conventional microscope and imaged on to the photo-cathode of an imaging photomultiplier tube where each single photon was counted. After time and space correlation, the waveform of the exciting signals can be displayed on a screen similar to a conventional electrical oscilloscope. Collaborative DEI fault analysis was conducted at MuAnalysis Inc. in cooperation with the NRC (National Research Council) and has resulted in one paper submitted to CSTC. 


\subsubsection{Advantages Over Existing $\Delta \Sigma$ FD Designs}

When used alone as a frequency demodulator, the new design has increased sensitivity to small deviation FM signals and superior SNR compared to previous $\Delta \Sigma$ FDs.

When used in a frequency synthesizer as shown in Fig.1.2, the $\Delta \Sigma$ FD enables the loop filter to be implemented digitally and made programmable. This makes the transfer function of the filter well defined and eliminates the need for large passive components.

Phase noise performance indicates a state of the art in-band BFM of $-200 @ 20 \mathrm{kHz}$ offset.

\subsection{Future Work}

Full testing of the third fabrication is currently underway. A custom-made PCB (Printed Circuit Board) will be manufactured to facilitate the testing.

DEI measurements will be continued on the third fabrication. The results from the third fabrication will be compared against those from the second fabrication in order to improve the technique.

The PFD and the CP are the dominant contributors to low frequency noise limiting the achievable SNR. Future work could be done to reduce the noise of these components. Once performance is optimized, the discriminator could be incorporated into a frequency synthesizer. 


\section{References}

[Bax94] W. Bax, "A sigma-delta frequency discriminator based synthesizer," Master's thesis, Carleton University, Ottawa ON, Canada, 1994.

[BC94] R. Beards and M. Copeland, "An oversampling delta-sigma frequency discriminator," Circuits and Systems -II: Analog and Digital Signal Processing, IEEE Transactions On, vol. 41, Jan 1994.

[BF95] R. Baird and T. Fiez, "Linearity enhancement of multi-bit delta-sigma a/d and d/a converters using data weighted averaging," Circuits and Systems-II:Analog and Digital Signal Processing, IEEE Transactions On, vol. 42, December 1995.

[Cra94] J. A. Crawford, Frequency synthesizer design handbook. Artech House, INC, 1994.

[CT92] J. Candy and G. Temes, Oversampling Delta-Sigma Data Converters. IEEE Press, 1992.

[FFRC07] J. Fang, N. Filiol, T. Riley, and M. Copeland, "A delta-sigma frequency discriminator with fractional-n divider and three-bit quantizer," in Circuit and Sys- 
tems, ISCAS'2007, 2007 IEEE International Symposium On, vol. TBD, 28-31 May 2007.

[FV04] P. Figueiredo and J. Vital, "Low kickback noise techniques for cmos latched comparators," Circuits and Systems, 2004. ISCAS'04 Proceedings of the 2004 International Symposium on, vol. 1, May 2004.

[HBL01] M. Hershenson, S. Boyd, and T. Lee, "Optimal design of a cmos op-amp via geometric programming," Computer-Aided Design of Integrated Circuits and Systems, IEEE Transactions on, vol. 20, Jan 2001.

[HFSN04] J. Hulse, J. Frank, and M. Simard-Normandin, "Dei as a performance and defect analysis tool," in Circuit and Systems, NEWCAS 2004. The 2nd Annual IEEE Northeast Workshop on, vol. 1, 20 June - 23 June 2004.

$\left[\mathrm{HSF}^{+}\right.$07] J. Hulse, K. Sarault, J. Fang, N. Filiol, and M. Simard-Normandin, "Dynamic electroluminescence imaging (dei) debugging of $0.18 \mu \mathrm{m}$ circuitry," in Canadian Semicondutor Technology Conference, 14 Aug - 17 Aug 2007.

[IM62] H. Inose and J. Murakami, "A telemeering system by code modulation - deltasigma modulation," in Space Electron. Telemetry.IEEE Transaction On, vol. SET-8, Sep 1962.

[NH96] O. Nys and R. Henderson, "An analysis of dynamic element matching techniques in sigma-delta modulation," Circuit and Systems, ISCAS'96 IEEE International Symposium On, vol. 1, May 1996.

[NST96] S. R. Norsworthy, R. Schreier, and G. C. Temes, Delta-Sigma Data Converters: Theory, Design, and Simulation. Wiley-IEEE Press, Oct 1996. 
[RFDK03] T. A. Riley, N. M. Filiol, Q. Du, and J. Kostamovaara, "Techniques for in-band phase noise reduction in delta-sigma synthesizers," Circuits and Systems -II: Analog and Digital Signal Processing, IEEE Transactions On, vol. 50, Nov 2003.

[Rod96] M. S. Roden, Analog And Digtial Communication Systems. Prentice-Hall, Inc., 1996.

[Roh83] U. L. Rohde, Digital PLL Frequency Synthesizers, Theory and Design. Prentice-Hall, Inc., 1983.

[WE02] N. H. Weste and K. Eshraghian, Principles of CMOS VLSI Design, 2nd ed. Addison-Wesley Publishing Company, 2002.

[YES92] G. Yin, F. Eynde, and W. Sansen, "A high-speed cmos comparator with 8-b resolution," Solid-State Circuits, IEEE Journal of, vol. 27, Feb 1992.

[Yuk85] A. Yukawa, "A cmos 8-bit high-speed a/d converter ic," Solid-State Circuits, IEEE Journal of, vol. sc-20, June 1985. 